\title{
FULL ABSTRACTION FOR FAIR TESTING IN CCS (EXPANDED VERSION) *
}

\author{
TOM HIRSCHOWITZ
}

CNRS, Université de Savoie

e-mail address: tom.hirschowitz@univ-savoie.fr

Abstract. In previous work with Pous, we defined a semantics for CCS which may both be viewed as an innocent form of presheaf semantics and as a concurrent form of game semantics. We define in this setting an analogue of fair testing equivalence, which we prove fully abstract w.r.t. standard fair testing equivalence.

The proof relies on a new algebraic notion called playground, which represents the 'rule of the game'. From any playground, we derive two languages equipped with labelled transition systems, as well as a strong, functional bisimulation between them.

2012 ACM CCS: [Theory of computation]: Models of computation-Concurrency-Process calculi;Semantics and reasoning-Program semantics-Denotational semantics/Catgorical semantics.

Key words and phrases: Programming languages; categorical semantics; presheaf semantics; game semantics; concurrency; process algebra.

* An extended abstract of this paper has appeared in CALCO '13.

Partially funded by the French ANR projets blancs PiCoq ANR-10-BLAN-0305 and Récré ANR-11-BS020010 .

네밈
(2) T. Hirschowitz Creative Commons 


\section{Contents}

1. Introduction

1.1. Overview of the approach

1.2. Main result: which behavioural equivalence?

1.3. Plan and overview

1.4. Related work

2. Prerequisites and preliminaries

2.1. Sets, categories, presheaves

2.2. Transition systems

2.3. CCS

3. Summary of previous work

3.1. Diagrams

3.2. From diagrams to moves

3.3. From moves to plays

3.4. Behaviours and strategies

3.5. Semantic fair testing

4. Playgrounds: from behaviours to strategies

4.1. Motivation: a pseudo double category

4.2. Behaviours

4.3. More axioms

4.4. Views

4.5. From behaviours to strategies

5. Playgrounds: transition systems

5.1. A syntax for strategies

5.2. The labelled transition system for strategies

5.3. Process terms

5.4. The labelled transition system for process terms

5.5. Translation and a first correctness result

6. Graphs and fair morphisms

6.1. Graphs with complementarity

6.2. Modular graphs and fair testing equivalence

6.3. Adequacy

6.4. Trees

6.5. Main result

7. CCS as a playground 62

7.1. A pseudo double category $\quad 62$

7.2. Correctness 63

7.3. CCS as a pre-playground 66

7.4. Towards CCS as a playground 71

7.5. CCS as a playground 73

8. Conclusion and perspectives $\quad 76$

8.1. Conclusion 76

8.2. Perspectives $\quad 76$

References 


\section{INTRODUCTION}

This paper is about game semantics for CCS [43]. Game semantics is originally a very successful approach to sequential denotational semantics [45, 26, 2]. Its basic idea is to interpret programs as strategies for a player in a game, and the computational environment as an opponent. Composition of programs is handled by letting the corresponding strategies interact. We

\begin{tabular}{c|c} 
Games & Concurrency \\
\hline position & configuration \\
player & agent \\
move & action \\
play & trace
\end{tabular}
mostly use game semantical terminology in this paper, but the above dictionary may help the intuition of concurrency theorists.

Denotational models of CCS are extremely diverse, and treat various behavioural equivalences, as surveyed by Winskel and Nielsen [54]. The closest game semantical work seems to be Laird's model [33], which achieves full abstraction w.r.t. trace (a.k.a. may testing) equivalence for a fragment of $\pi$. The goal of the present paper is to design the first game semantics for a finer equivalence than trace equivalence, in the simpler setting of CCS (we plan to address the full $\pi$-calculus in future work). The reason Laird is limited to trace equivalence is that the standard notion of strategy is a set of plays (with well-formedness conditions). Hence, e.g., the famous coffee machines, $a . b+a . c$ and $a .(b+c)$, are identified. Following two recent, yet independent lines of work [49, 24], we generalise strategies by allowing them to accept plays in several ways, thus reconciling game semantics with presheaf models [30]. Winskel et al.'s approach is only starting to be applied to concrete languages, see for example the work in progress on an affine, concurrent variant of Idealised Algol [8]. The approach of [24, 25] ( $\mathrm{HP})$ was used to give a game semantics for CCS, and define a semantic analogue of fair testing equivalence, but no adequacy result was proved. We here prove full abstraction of semantic fair testing equivalence w.r.t. standard fair testing equivalence. Our model is compositional, since (1) all syntactic constructs of CCS have natural interpretations, and (2) global dynamics may be inferred from local dynamics, as in any game semantics (see the paragraph on innocence below and Sections 3.4.2 and 3.4.3).

\subsection{Overview of the approach.}

Truly concurrent plays. First of all, as in [49], our notion of play is truly concurrent. Indeed, it does not keep track of the order in which (atomic) moves occur. Instead, it only retains causal dependencies between them (see Section 3.3). Furthermore, our plays form a proper category, which enables in particular a smooth treatment of bound variables. Briefly, plays that differ only up to a permutation of channels are isomorphic, and by construction strategies handle them correctly.

Branching behaviour. Second, we deal with branching behaviour. Standardly, and ignoring momentarily the previous paragraph, a strategy is essentially a prefix-closed set of 'accepted' plays. This is equivalent to functors $\mathbb{E}^{o p} \rightarrow 2$, where $\mathbb{E}$ is the poset of plays ordered by prefix inclusion, and 2 is the poset $0 \leq 1$ (E stands for 'extension'). A play $u$ is 'accepted' by such a functor $F$ when $F(u)=1$, and if $u^{\prime} \leq u$, then functoriality imposes that $F(u) \leq F\left(u^{\prime}\right)$, hence $F\left(u^{\prime}\right)=1$ : this is prefix-closedness. In order to allow plays to be accepted in several ways, we follow presheaf models [30] and move to functors $\mathbb{E}^{o p} \rightarrow$ set, where set is the category 
of finite ordinals and all functions between them 1 . Thus, to each play $u \in \mathbb{E}$, a strategy associates a set of ways to accept it, empty if $u$ is rejected. E.g., in the simplistic setting where $\mathbb{E}$ denotes the poset of words over actions, ordered by prefix inclusion, the coffee machine $a . b+a . c$ is encoded as the presheaf $S$ defined on the left and pictured on the right:

- $S(\epsilon)=\{\star\}$,

- $S(a)=\left\{x, x^{\prime}\right\}$,

- $S(a b)=\{y\}$,

- $S(a c)=\left\{y^{\prime}\right\}$,
- $S$ empty otherwise,

- $S(\epsilon \hookrightarrow a)=\left\{x \mapsto \star, x^{\prime} \mapsto \star\right\}$,

- $S(a \hookrightarrow a b)=\{y \mapsto x\}$,

- $S(a \hookrightarrow a c)=\left\{y^{\prime} \mapsto x^{\prime}\right\}$,

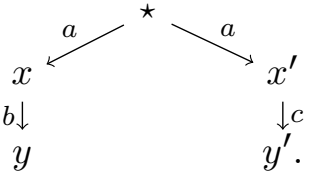

This illustrates what is meant by 'accepting a play in several ways': the play $a$ is here accepted in two ways, $x$ and $x^{\prime}$. The other coffee machine is of course obtained by identifying $x$ and $x^{\prime}$. In our setting, plays are considered relative to their initial position $X$, hence strategies are presheaves $\mathbb{E}_{X}^{o p} \rightarrow$ set on the category of plays over $X$.

Innocence. Finally, defining strategies as presheaves on plays is too naive, which leads us to reincorporate the game semantical idea of innocence. Example 3.14 below exhibits such a presheaf in which two players synchronise on a public channel $a$, without letting others interfere. In CCS, this would amount to a process like $\bar{a} . P|a . Q| a . R$ in which, say, the first two processes could arrange for ruling out the third. Considering such presheaves as valid strategies would break our main result.

In the Hyland-Ong approach, innocent strategies may be defined as prefix-closed sets of views, where views are special plays representing the information that a player may 'access' during a global play. The global strategy $\bar{S}$ associated to an innocent strategy $S$ is then recovered by decreeing that $\bar{S}$ accepts all plays whose views are accepted by $S$. This leads us to consider a subcategory $\mathbb{E}^{\mathbb{V}}$ of the category $\mathbb{E}$ of plays, whose objects are called views. We thus have for each position $X$ two categories of strategies: the naive one, the category $\left[\mathbb{E}_{X}^{o p}\right.$, set $]$ of behaviours on $X$, consists of presheaves on plays; the more relevant one, the category $\left[\left(\mathbb{E}_{X}^{\mathbb{V}}\right)^{o p}\right.$, set] of strategies on $X$, consists of presheaves on views.

How, then, do we recover the global behaviour associated to a strategy, which is crucial for defining our semantic fair testing equivalence? The right answer is given by a standard categorical construction called right Kan extension (see Section 3.4.2). Roughly, for the behaviour $B_{S}$ associated to a strategy $S$, a way to accept some play $u \in \mathbb{E}_{X}$ is a compatible family of ways for $S$ to accept all views of $u$. In the boolean, setting (considering functors $\mathbb{E}_{X}^{o p} \rightarrow 2$ ), this reduces to $B_{S}$ accepting $u$ iff all its views are accepted by $S$. Our definition thus generalises Hyland and Ong's.

Finally, game semantical parallel composition (different from CCS parallel composition, though inspired from it) intuitively lets strategies interact together. We account for it as follows. If we partition the players of a play $X$ into two teams, we obtain two subpositions $X_{1} \hookrightarrow X \hookleftarrow X_{2}$, each player of $X$ belonging to $X_{1}$ or $X_{2}$ according to its team. We have that the category $\mathbb{E}_{X}^{\mathbb{V}}$ of views on $X$ is isomorphic to the coproduct category $\mathbb{E}_{X_{1}}^{\mathbb{V}}+\mathbb{E}_{X_{2}}^{\mathbb{V}}$. The parallel composition of any two

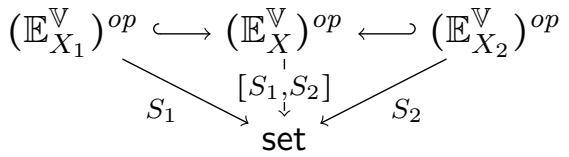
strategies $S_{1}$ and $S_{2}$ on $X_{1}$ resp. $X_{2}$ is simply obtained by universal property of coproduct, as above right.

\footnotetext{
${ }^{1}$ The author learnt this point of view from a talk by Sam Staton.
} 
1.2. Main result: which behavioural equivalence? With our game in place, we easily define a translation of CCS processes into strategies. It then remains to demonstrate the adequacy of this translation. Our strategies are actually rather intensional, so we cannot hope for adequacy w.r.t. equality of strategies. Instead, we exploit the rich structure of our model to define both an LTS and an analogue of fair testing equivalence on the semantic side, i.e., for strategies. We then provide two results. The most important, in the author's view, is full abstraction w.r.t. standard fair testing semantics (Corollary 6.51). But the second result might be considered more convincing by many: it establishes that our semantics is fully abstract w.r.t. weak bisimilarity (Corollary 6.50). A reason why the latter result is here considered less important originates in the tension between LTS semantics and reduction semantics [47]. Briefly, reduction semantics is simple and intuitive, but it operates on equivalence classes of terms (under so-called structural congruence). On the other hand, designing LTSs is a subtle task, rewarded by easier, more structural reasoning over reductions. We perceive LTS semantics as less intrinsic than reduction semantics. E.g., for more sophisticated calculi than CCS, several LTSs exist, which yield significantly different notions of bisimilarity.

Beyond LTS-based equivalences, we see essentially two options: barbed congruence [52] or some testing equivalence [9]. Barbed congruence equates processes $P$ and $Q$, roughly, when for all contexts $C$,

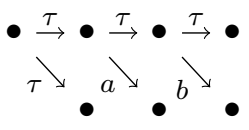
$C[P]$ and $C[Q]$ are weakly bisimilar w.r.t. reduction (i.e., only $\tau$-actions are allowed), and furthermore they have the same interaction capabilities at all stages. Barbed congruence is sometimes perceived as too discriminating w.r.t. guarded choice. Consider, e.g., the CCS process $P_{1}$ pictured above, and let $P_{2}$ be the same with $a$ and $b$ swapped. Both processes may disable both actions $a$ and $b$, the only difference being that $P_{1}$ disables a before disabling $b$. Barbed congruence distinguishes $P_{1}$ from $P_{2}$ (take $C=\square \mid \bar{a}$ ), which some view as a deficiency.

Another possibility would be must testing equivalence [9]. Recall that $P$ must pass a test process $R$ iff all maximal executions of $P \mid R$ perform, at some point, a fixed 'tick' action [19], here denoted by $\nabla$. Then, $P$ and $Q$ are must testing equivalent iff they must pass the same tests. Must testing equivalence is sometimes perceived as too discriminating w.r.t. divergence. E.g., consider $Q_{1}=! \tau \mid a$ and $Q_{2}=a$. Perhaps surprisingly, $Q_{1}$ and $Q_{2}$ are not must testing equivalent. Indeed, $Q_{2}$ must pass the test $\bar{a} . \odot$, but $Q_{1}$ does not, due to an infinite, silent reduction sequence.

We eventually go for fair testing equivalence, which was originally introduced (for CCSlike calculi) to rectify both the deficiency of barbed congruence w.r.t. choice and that of must testing equivalence w.r.t. divergence. The idea is that two processes are equivalent when they should pass the same tests. A process $P$ should pass the test $T$ iff their parallel composition $P \mid T$ never loses the ability of performing the special 'tick' action, after any tick-free reduction sequence. Fair testing equivalence thus equates $P_{1}$ and $P_{2}$ above, as well as $Q_{1}$ and $Q_{2}$. Cacciagrano et al. [7] provide an excellent survey.

1.3. Plan and overview. We now give a bit more detail on the contents. In Section 2, we introduce our notations and some preliminaries. Section 3 summarises from HP the game for CCS, the notions of strategy and behaviour, the translation (-) of CCS processes into strategies, and semantic fair testing equivalence. The rest is devoted to proving that $(-)$, here decomposed as $\llbracket-\rrbracket \circ \theta$ (see below), is such that $P \sim_{f, s} Q$ iff $(P) \sim_{f}(Q)$, where $\sim_{f, s}$ is standard fair testing equivalence (Corollary 6.51). 
1.3.1. Playgrounds. Our proof of this result takes a long detour to introduce a new algebraic gadget called playground, which we now motivate. Our first attempts at proving the full abstraction result were obscured by a tight interleaving of

- results stating common properties of moves in the game, or of plays, and

- results and constructions on strategies derived from those (e.g., the LTs for strategies).

On the other hand, the reasons why our constructions work are intuitively simple. Namely, innocent strategies essentially amount to describing syntax trees by selecting their branches amongst a set of all possible branches. This enlarges the universe of terms slightly, but in game semantics, one studies properties of terms which also make sense for such generalised terms. Compositionality and the definition of our semantic fair testing equivalence are examples where using strategies instead of terms tends to simplify the constructions. E.g., associated behaviours are recovered from innocent strategies through Kan extension, thanks to an expressive notion of morphism between plays. Our results essentially follow from this correspondence between terms and strategies.

Example 1.1. To illustrate what we mean by generalised terms, consider standard, unlabelled binary trees as a stripped down example of a term language. Such trees admit a description as prefix-closed sets of words over $\{0,1\}$ (their sets of occurrences). In order to get exactly trees, such sets should be constrained a bit. E.g., the empty set of words, or the set $\{(),(0)\}$ do not describe any tree.

Playgrounds are a first attempt at a general framework describing this correspondence between terms and strategies. We develop their theory in Sections 4 and 5, whose main result is a strong bisimulation between both presentations (i.e., terms vs. strategies). This is then expoited in the next sections to derive the main results.

The basis for playgrounds are pseudo double categories [20, 21, 35, 17], a weakening of Ehresmann's double categories [11, 12]. Playgrounds are thus pseudo double categories with additional structure. The objects of a playground represent positions in the game. There are two kinds of morphisms: vertical morphisms represent plays, while horizontal ones represent embeddings of positions. E.g., there are special objects representing 'typical' players; and a player of a position $X$ is a horizontal morphism $d \rightarrow X$ from such a typical player, in a Yoneda-like way. There are then axioms to model atomicity (plays may be decomposed into atomic moves) and locality (plays over a large position may be restricted to any subposition; each player only sees part of the play). There are finally a few more technical axioms.

In Section 4, we give the definition and derive a few basic results and constructions. In particular, we define a naive notion of strategy, behaviours, and a less naive notion, strategies. Finally, we relate the two by exhibiting a functor from strategies to behaviours. In Section 5, we prove that strategies are in bijective correspondence with infinite terms in a certain language. We then derive from this an LTS $\mathcal{S}_{\mathbb{D}}$ for strategies. Furthermore, we define a second language, which is closer to usual process calculi. And indeed, instantiating this general language to our game for CCS yields essentially CCS, the only difference being that channel creation is treated on an equal footing with input and output. We further equip this language of process terms with an LTS $\mathcal{T}_{\mathbb{D}}$. Finally, we define a translation from process terms to strategies $\llbracket-\rrbracket: \mathcal{T}_{\mathbb{D}} \rightarrow \mathcal{S}_{\mathbb{D}}$, which is proved to be a strong bisimulation (Theorem 5.35).

At this point, it remains

(1) to show that the pseudo double category $\mathbb{D}^{C C S}$ formed by our game does satisfy the axioms for playgrounds, and

(2) to use the strong bisimulation $\llbracket-\rrbracket$ to derive our main results. 
1.3.2. Graphs with complementarity. We start with (2), because we feel doing otherwise would disrupt the flow of the paper. Indeed, it should not be surprising at all that $\mathbb{D}^{C C S}$ forms a playground; and furthermore the methods employed to show this are in sharp contrast with the rest of the paper. The plan for (2), carried out in Section 6, is as follows.

First, we reduce semantic fair testing equivalence to fair testing equivalence in the LTS $\mathcal{S}_{\mathbb{D} C C S}$, thus bridging the gap between the game semantical world and LTSs. But this is not as simple as it looks. Indeed, Hennessy and De Nicola's original setting for testing equivalences [9] is not quite expressive enough for our purposes, which leads us to define a slightly more general one, called modular graph with complementarity. First, our setting is 'typed', in the sense that not all tests may be applied to a process $P$, only tests of a type 'compatible' with $P$. Furthermore, in modular graphs with complementarity, fair testing equivalence relies on a notion of complementarity saying when two transitions may be glued together to form a closed-world transition. Thus, fair testing equivalence is 'intrinsic', i.e., does not depend on any alphabet. So we have a mere LTS $\mathcal{S}_{\mathbb{D} C C S}$ over an ad hoc alphabet $\mathbb{Q}$ derived from $\mathbb{D}^{C C S}$, and we need promote it into a modular graph with complementarity. This goes by refining the original alphabet $\mathbb{Q}$ with 'interfaces', yielding a new alphabet $\mathbb{I} \mathbb{Q}$. We then define a morphism $\chi: \mathbb{I} \rightarrow \mathbb{Q}$, and pull $\mathcal{S}_{\mathbb{D} C C S}$ back along $\chi$, thus obtaining our modular graph with complementarity $\mathcal{S}_{\mathbb{D}}^{\mathbb{Q}} C C S$ (which is thus also an LTS over $\mathbb{I} \mathbb{Q}$ ). In passing, we do the same for $\mathcal{T}_{\mathbb{D} C C S}$, which yields $\mathcal{T}_{\mathbb{D} C C S}^{\mathbb{Q}}$ : this will be useful later. We finally prove that fair testing equivalence in $\mathcal{S}_{\mathbb{D} C C S}^{\mathbb{Q}}$ coincides with semantic fair testing equivalence (Lemma 6.26). Similarly, we construct a modular graph with complementarity $C C S$ for CCS, and show that fair testing equivalence therein coincides with standard fair testing equivalence (Proposition 6.21). We are thus reduced to proving that some composite

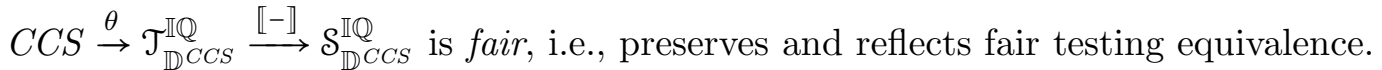

Our second step is to establish a sufficient condition for a relation $R: G \rightarrow H$ to be fair and to apply this to the graph of our translation $C C S \rightarrow \mathcal{S}_{\mathbb{D} C C S}^{\mathbb{I}}$. The idea is to define what an adequate alphabet $A$ should be in our setting, and to prove that, essentially, if we can find an adequate alphabet $A$ for $G$ and $H$, such that $R$ is a relation over $A$, then $R$ is fair as soon as

- $R$ is included in weak bisimilarity over $A$, and

- both graphs have enough $A$-trees, in a sense inspired by the notion of failure [48].

In order to apply this, we transform $\mathcal{S}_{\mathbb{D}^{C C S}}^{\mathbb{Q}}$ and $\mathcal{T}_{\mathbb{D}^{C C S}}^{\mathbb{Q}}$ into modular graphs with complementarity over the same alphabet $\mathbb{A}$ (i.e., set of labels) as $C C S$. We proceed by 'relabeling' along some morphism of graphs $\mathbb{I} \mathbb{Q} \stackrel{\xi}{\rightarrow} \mathbb{A}$. We still have our translation $\mathcal{T}_{\mathbb{D} C C S}^{\mathbb{Q}} \stackrel{\llbracket-\rrbracket}{\longrightarrow} \mathcal{S}_{\mathbb{D}^{C C S}}^{\mathbb{Q}}$, which is a strong, functional bisimulation over $\mathbb{A}$. It thus remains to check that (a) the map $C C S \stackrel{\theta}{\rightarrow} \mathcal{T}_{\mathbb{D} C C S}^{\mathbb{Q}}$ is included in weak bisimilarity, and (b) both $C C S$ and $\mathcal{S}_{\mathbb{D}}^{\mathbb{Q}}$ Q $\mathbb{A}$-trees. Roughly, $G$ has enough $A$-trees when, for any $t$ in a certain class of tree-like LTSs over $A$ called $A$-trees, there exists $x_{t} \in G$ weakly bisimilar to $t$. For (b), all three LTss under consideration clearly have enough $\mathbb{A}$-trees. For (a), our proof is brute force.

1.3.3. CCS as a playground. We finally deal in Section 7 with the last missing bit of our proof: we show that $\mathbb{D}^{C C S}$ forms a playground. This rests upon the following two main ingredients. 
First, we design a correctess criterion for plays, in a sense close to correctness criteria in linear logic. Namely, plays from some position $X$ to position $Y$ are represented as particular cospans $Y \stackrel{s}{\rightarrow} U \stackrel{t}{\leftarrow} X$ in some category. Specifically, they are obtained by closing a given set of cospans named moves under identities and composition. We design a combinatorial criterion for deciding when an arbitrary cospan is indeed a play.

The second main ingredient is a construction of the restriction of a play $U$ from some position $X$ to a subposition $X^{\prime} \hookrightarrow X$. Briefly, this means computing the part of $U$ which is relevant to players in $X^{\prime}$. This construction is almost easy: most of $U$ may be 'projected' back onto the initial position $X$, and then a mere pullback

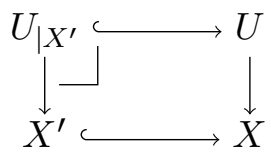

of sets gives the needed restriction. The glitch is that in general some parts of $U$ may not canonically be projected back onto $X$. The principle for this projection is as simple as: project, e.g., input moves to the inputting player. The problem arises for synchronisations. Projecting them to the channel over which the synchronisation occurs does not yield the desired result, and similarly projecting to either of the involved players fails. Our solution is to ignore synchronisations at first, and later reintroduce them automatically using a technique from algebraic topology: factorisation systems [28].

With both of these ingredients in place, the proof is relatively straightforward.

Section 8 concludes and provides some perspectives for future work.

1.4. Related work. Our bisimulation result relating terms to strategies for any playground draws inspiration from Kleene coalgebra [ $[, 6]$. There, the main idea is that both the syntax and the semantics of various kinds of automata should be derived from more basic data describing, roughly, the 'rule of the game'. Formally, starting from a well-behaved (polynomial) endofunctor on sets, one constructs both (1) an equational theory and (2) a sound and complete coalgebraic semantics. This framework has been applied in standard automata theory, as well as in quantitative settings. Nevertheless, its applicability to programming language theory is yet to be established. E.g., the derived languages do not feature parallel composition. Our playgrounds may be seen as a first attempt to convey such ideas to the area of programming language theory. Technically, our framework is rather different though, in that we replace the equational theory by a transition system, and the coalgebraic semantics by a game semantics. To summarise, our approach is close in spirit to Kleene coalgebra, albeit without quantitative aspects. Conversely, Kleene coalgebra resembles our approach without innocence.

Building upon previous work [1, 40, 42] on asynchronous games, a series of papers by Winskel and collaborators (see, e.g., Rideau and Winskel [49], Winskel [53]) attempt to define a notion of concurrent strategy encompassing both innocent game semantics and presheaf models. Ongoing work evoked above [8] shows that the model does contain innocent game semantics, but presheaf models are yet to be investigated. (Their notion of innocence, borrowed from Faggian and Piccolo [13], is not intended to be related to that of Hyland and Ong.) In their framework, a game is an event structure, whose events are thought of as moves, equipped with a notion of polarity. In one of the most recent papers in the series [53], Winskel establishes a strong relationship between his concurrent strategies and presheaves. 
For a given event structure with polarity $A$, he considers the so-called $S$ cott order on the set $\mathcal{C}(A)$ configurations of $A$. For two configurations $c$ and $d$, we have $c \varsigma_{A} d$ iff $d$ may be obtained from $c$ by removing some negative moves and then adding some positive ones, in a valid way. Strategies are then shown to coincide with presheaves on $\left(\mathcal{C}(A), \varsigma_{A}\right)$. This is close in spirit to our use of presheaves, but let us mention a few differences. First, our games do not directly deal with polarity. Furthermore, in our setting, for any morphism $p \rightarrow q$ of plays, $q$ is intuitively bigger than $p$ in some way, unlike what happens with the Scott ordering. Finally, an important point in our use of (pre)sheaves is that, unlike configuration posets, our plays form proper categories, i.e., homsets may contain more than one element (intuitively, the same view may have several occurrences in a given play). Thus, potential links between both approaches remain to be further investigated.

To conclude this paragraph, let us mention a few, more remotely related lines of work. Melliès [41], although in a deterministic and linear setting, incorporates some 'concurrency' into plays by presenting them as string diagrams. Our notion of innocent strategy shares with Harmer et al.'s [23] presentation of innocence based on a distributive law the goal of better understanding the original notion of innocence. Finally, others have studied game semantics in non-deterministic [22] or concurrent [18, 33] settings, using coarser, trace-based behavioural equivalences.

\section{Prerequisites AND PREliminaries}

In this section, we recall some needed material and introduce our notations. We attempt to provide intuitive, yet concise explanations, but these may not suffice to get the non-specialist reader up to speed, so we also provide references when possible.

For the reader's convenience, we finally provide in Figure 6 (end of paper) a summary of notations, beyond those introduced here.

2.1. Sets, categories, presheaves. We make intensive use of category theory, of which we assume prior knowledge of categories, functors, natural transformations, limits and colimits, adjoint functors, presheaves, bicategories, Kan extensions, and pseudo double categories. All of this except pseudo double categories is entirely covered in Mac Lane's standard textbook [38] and the beginning of Mac Lane and Moerdijk [39]. For a more leisurely introduction, one may consult Lawvere and Schanuel [34], or Leinster [36]. The needed material on Kan extensions roughly amounts to their expression as ends, which is recalled when used (Section 3.4.2). The last bit, namely the notion of pseudo double category is briefly recalled below, after fixing some notation. Finally, there are very local uses of locally presentable categories [3] in the present section, and of adhesive category theory [32] in the proof of Lemma 7.35.

Throughout the paper, any finite ordinal $n$ is seen as $\{1, \ldots, n\}$ (rather than $\{0, \ldots, n-$ $1\})$. In any category, for any object $C$ and set $X$, let $X \cdot C$ denote the $|X|$-fold coproduct of $C$ with itself, i.e., $C+\cdots+C,|X|$ times.

Set is the category of sets; set is a skeleton of the category of finite sets, e.g., the category of finite ordinals and arbitrary maps between them; ford is the category of finite ordinals and monotone maps between them. For any category $\mathbb{C}, \widehat{\mathbb{C}}=\left[\mathbb{C}^{o p}\right.$, Set $]$ denotes the category of presheaves on $\mathbb{C}$, while $\overline{\mathbb{C}}=\left[\mathbb{C}^{o p}\right.$, set $]$ and $\widehat{\mathbb{C}}=\left[\mathbb{C}^{o p}\right.$, ford $]$ respectively denote the categories of presheaves of finite sets and of finite ordinals. One should distinguish, e.g., 'presheaf of finite sets' $\mathbb{C}^{o p} \rightarrow$ set from 'finite presheaf of sets' $F: \mathbb{C}^{o p} \rightarrow$ Set. The category 
$f$ of finite presheaves is the full subcategory of $\widehat{\mathbb{C}}$ spanning presheaves $F$ which are finitely presentable [3]. In presheaf categories, finitely presentable objects are the same as finite colimits of representables. In the only case we will use ( $\mathbb{C}$ below), because representables have finite categories of elements, the latter in turn coincide with presheaves $F$ such that the disjoint union $\sum_{c \in \mathrm{ob}(\mathbb{C})} F(c)$ is finite. For all presheaves $F$ of any such kind, $x \in F(d)$, and $f: c \rightarrow d$, let $x \cdot f$ denote $F(f)(x)$.

Remark 2.1. This conflicts with the notation $X \cdot C$ above, but context should disambiguate, as in $X \cdot C$ a set $X$ acts on an object $C$, whereas in $x \cdot f$, a morphism $f$ acts on an object $x$.

We denote the Yoneda embedding by $\mathrm{y}: \mathbb{C} \rightarrow \widehat{\mathbb{C}}$, and often abbreviate $\mathrm{y}(c)$ to just $c$.

For any functor $F: \mathbb{C} \rightarrow \mathbb{D}$ and object $D \in \mathbb{D}$, let $F_{D}$ denote the comma category on the left below, and $F(D)$ denote the pullback category on the right:
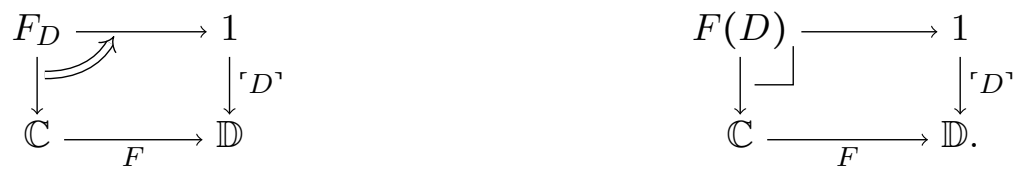

When $F$ is clear from context, we simply write $\mathbb{C}_{D}$, resp. $\mathbb{C}(D)$. Also, as usual, when $F$ is the identity, we use the standard slice notation $\mathbb{D} / D$.

Finally, we briefly recall pseudo double categories. They are a weakening of Ehresmann's double categories [11, 12], notably studied by Grandis and Paré [20, 21], Leinster [35], and Garner [17]. The weakening lies in the fact that one dimension is strict and the other weak (i.e., bicategory-like). We need to consider proper pseudo double categories, notably we use cospans in examples, but we often handle pseudoness a bit sloppily. Indeed, the proofs of Section 4 quickly become unreadable when accounting for pseudoness.

A pseudo double category $\mathbb{D}$ consists of a set ob $(\mathbb{D})$ of objects, shared by a 'horizontal' category $\mathbb{D}_{h}$ and a 'vertical' bicategory $\mathbb{D}_{v}$. Following Paré [46], $\mathbb{D}_{h}$, being a mere category, has standard notation (normal arrows, o for composition, id for identities), while the bicategory $\mathbb{D}_{v}$ earns fancier notation $\left(\rightarrow\right.$ arrows, $\bullet$ for composition, $i d^{\bullet}$ for identities).

is furthermore equipped with a set of double cells $\alpha$, which have vertical, resp. horizontal, domain and codomain, denoted by $\operatorname{dom}_{v}(\alpha), \operatorname{cod}_{v}(\alpha), \operatorname{dom}_{h}(\alpha)$, and $\operatorname{cod}_{h}(\alpha)$.

We picture this as, e.g., $\alpha$ on the right, where $u=\operatorname{dom}_{h}(\alpha), \quad X \stackrel{h}{\longrightarrow} X^{\prime} \stackrel{k}{\longrightarrow} X^{\prime \prime}$ $u^{\prime}=\operatorname{cod}_{h}(\alpha), h=\operatorname{dom}_{v}(\alpha)$, and $h^{\prime}=\operatorname{cod}_{v}(\alpha)$. Finally, there are $u \downarrow \stackrel{\alpha}{\Rightarrow} u^{\prime} \stackrel{\alpha^{\prime}}{\Rightarrow} b^{\prime \prime}$ operations for composing double cells: horizontal composition - composes them along a common vertical morphism, vertical composition • composes along horizontal morphisms. Both vertical compositions (of morphisms and of double cells) may be associative only up to coherent isomorphism. The full axiomatisation is given by Garner [17], and we here only mention the interchange law, which says that the two ways of parsing the above diagram coincide: $\left(\beta^{\prime} \circ \beta\right) \bullet\left(\alpha^{\prime} \circ \alpha\right)=\left(\beta^{\prime} \bullet \alpha^{\prime}\right) \circ(\beta \bullet \alpha)$.

For any (pseudo) double category $\mathbb{D}$, we denote by $D$ the category with vertical morphisms as objects and double cells as morphisms, and by $\mathbb{D}_{V}$ the bicategory with horizontal morphisms as objects and double cells as morphisms. Domain and codomain maps arrange into functors $\operatorname{dom}_{v}, \operatorname{cod}_{v}: D \rightarrow \mathbb{D}_{h}$ and $\operatorname{dom}_{h}, \operatorname{cod}_{h}: \mathbb{D}_{V} \rightarrow \mathbb{D}_{v}$. We will refer to $\operatorname{dom}_{v}$ and $\operatorname{cod}_{v}$ simply as dom and cod, reserving subscripts for $\operatorname{dom}_{h}$ and $\operatorname{cod}_{h}$.

We introduce a bit more notation. 
Definition 2.2. A double cell is special when its vertical domain and codomain are (horizontal) identities.

For any object $X \in \mathrm{ob}(\mathbb{D}), D(X)$ denotes the category with

- objects all vertical morphisms to $X$, and

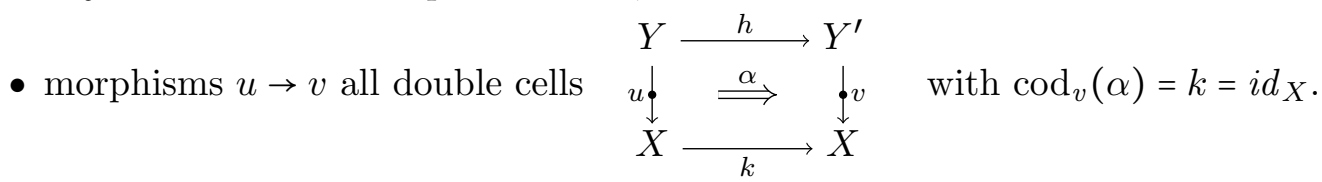

This complies with noting $\mathbb{C}(D)$ for the pullback category (2.1), taking $\operatorname{cod}_{v}$ for $F$ and $X$ for $D$.

2.2. Transition systems. Beyond category theory, this paper also makes heavy use of the theory of LTSs and associated techniques, especially bisimulation and other behavioural equivalences. The notion of LTS that we'll use here is a little more general than usual. Indeed, usually, the transitions of an LTS are labelled with letters in a given set called the alphabet, or the set of actions. Here, we consider the case where the vertices of an LTS may be typed, and actions may change the type. Extending the usual theory to this setting is straightforward, so we only provide a brief overview. For more on the usual theory, modern references are Sangiorgi 50] and Sangiorgi and Rutten [51]. Our setting is essentially a baby version of Fiore's [14] (see the references therein for precursors).

Let $G$ ph be the category of reflexive graphs, which has as objects diagrams $s, t: E \rightrightarrows V$ in Set, equipped with a further arrow $e: V \rightarrow E$ such that $s \circ e=t \circ e=i d_{V}$. We will as usual denote $e(v)$ by $i d_{v}$. Morphisms are those morphisms between underlying graphs which preserve identity arrows. Gph is thus the category of presheaves over the category $\star \longleftarrow_{t}^{s}[1]$ with $e \circ s=i d_{\star}$ and $e \circ t=i d_{\star}$.

Definition 2.3. For any $A \in \mathrm{Gph}$, let the category of LTS s over $A$ be just the slice category $\mathrm{Gph} / A$.

2.2.1. Basic notation. A is called the alphabet, which goes slightly beyond the usual notion of an alphabet. The latter would here come in the form of the graph with one vertex, an identity edge, plus an edge for each letter. By convention, and mainly to ease graphical intuitions in Sections 4 and 5, for any LTS $p: G \rightarrow A$, we understand an edge $e: x^{\prime} \rightarrow x$ in $G$ as a transition from $x$ to $x^{\prime}$. Of course, to recover a more standard notation, one may replace all graphs with their opposites. When $e$ does not matter, but $p(e)$ does, we denote such a transition by $x_{A} \stackrel{p(e)}{\longleftarrow} x^{\prime}$, omitting the subscript $A$ when clear from context.

For any reflexive graph $A$, we denote by $A^{\star}$ the graph with the same vertices and arbitrary paths as edges. $A^{\star}$ is reflexive, with identity edges given by empty paths. Similarly, $f^{\star}: A^{\star} \rightarrow B^{\star}$ is the morphism induced by $f: A \rightarrow B$. This defines a functor Gph $\rightarrow$ Cat, which is not left adjoint to the forgetful functor $U:$ Cat $\rightarrow$ Gph. There is a left adjoint, though, which we denote by fc. It is given by a quotient of $A^{\star}$, essentially equating $(i d)$ and (), i.e., the singleton, identity path and the empty one. 
Definition 2.4. Let $\mathrm{fc}(A)$ denote the graph with the same vertices as $A$, whose edges $x \rightarrow x^{\prime}$ are paths $x \rightarrow^{\star} x^{\prime}$ in $A$, considered equivalent modulo removal of identity edges.

Any path $\rho$ has a normal form, obtained by removing all identity edges and denoted by $\widetilde{\rho}$. We will deem such normal forms identity-free. We denote by $x \underset{A}{\stackrel{a}{=}} x^{\prime}$ any path $\rho: x^{\prime} \rightarrow^{\star} x$ in $G$, such that $\widetilde{p^{\star}(\rho)}=\widetilde{(a)}$. Concretely, if $a$ is an identity, then $p^{\star}(\rho)$ only consists of identity edges; otherwise, $p^{\star}(\rho)$ consists of $a$, possibly surrounded by identity edges. In the former case, we further abbreviate the notation to $x \Leftarrow x^{\prime}$ (observe that $\rho$ may well be empty). Similarly, for any path $r$ in $A^{\star}, x \underset{A}{\stackrel{r}{=}} x^{\prime}$ denotes any path $\rho: x^{\prime} \rightarrow^{\star} x$ in $G$ such that $\overline{p^{\star}(\rho)}=\widetilde{r}$.

2.2.2. Bisimulation and change of base. In this section, we revisit the usual notion of (strong and weak) bisimulation in our graph-based setting, and provide a few stability results under base change and cobase change. Let us start with strong bisimulations.

Definition 2.5. For any $G, G^{\prime} \in \mathrm{Gph}$, a morphism $f: G \rightarrow G^{\prime}$ is a graph fibration iff for all $x \in G, y \in G^{\prime}$, and $e^{\prime} \in G^{\prime}(y, f(x))$, there exist $x^{\prime} \in G$ and $e \in G\left(x^{\prime}, x\right)$ such that $f(e)=e^{\prime}$.

Consider morphisms $p: G \rightarrow A$ and $p^{\prime}: G^{\prime} \rightarrow A$. A relation over $A$ is a subgraph of the pullback

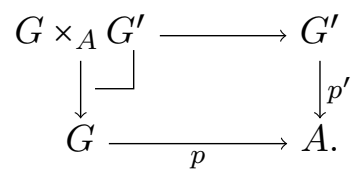

In particular, if two edges $\left(e, e^{\prime}\right)$ are related by some $R \subseteq G \times{ }_{A} G^{\prime}$, then so are their sources, resp. targets. We denote such relations by $R: G \rightarrow G^{\prime}$.

We will most often deal with full relations, i.e., such that $R\left(e, e^{\prime}\right)$ iff both sources and targets are related. Of course, such relations need only to be defined on vertices.

Definition 2.6. A simulation $G \longrightarrow G^{\prime}$ is a relation $R$ over $A$ such that for all $e \in G\left(x^{\prime}, x\right)$, if $R(x, y)$ then there exist $y^{\prime}$ and $e^{\prime} \in G^{\prime}\left(y^{\prime}, y\right)$ such that $R\left(e, e^{\prime}\right)$. A bisimulation is a simulation whose converse also is a simulation.

When $R$ is full, $R$ is a simulation iff for all $e \in G\left(x^{\prime}, x\right)$, if $R(x, y)$ then there exists $y^{\prime}$ and $e^{\prime} \in G^{\prime}\left(y^{\prime}, y\right)$ such that $R\left(x^{\prime}, y^{\prime}\right)$ and $e$ and $e^{\prime}$ are mapped to the same edge in $A$.

Proposition 2.7. $R$ is a simulation iff its first projection $R \rightarrow G \times{ }_{A} G^{\prime} \rightarrow G$ is a graph fibration. Accordingly, $R$ is a bisimulation iff both projections are graph fibrations.

Proof. Straightforward.

Remark 2.8. The characterisation of simulations in terms of graph fibrations may be attributed to Joyal et al. [30], who first observed that a morphism $f: G \rightarrow G^{\prime}$ in $G \mathrm{ph} / A$ is a functional bisimulation iff for any commuting square as the exterior of

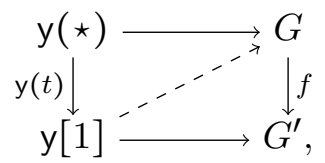

there exists a dashed arrow making both triangles commute. Here, $y(t): y(\star) \rightarrow y[1]$ maps the reflexive graph with a single vertex (and its identity edge) to the one with two vertices and just one non-identity edge $e$ between them, by picking out the target of $e$. This precisely says that $f$ is a graph fibration. 
A peculiar aspect of this characterisation is that it may seem independent from $A$. Actually, $R$ is a relation over $G \times{ }_{A} G^{\prime}$, and $f$ is a morphism over $A$.

As usual, fixing $G$ and $G^{\prime}$ over $A$, we have:

Proposition 2.9. Bisimulations are closed under union, and the union of all bisimulations, called bisimilarity, is again a bisimulation, the maximum one.

Considering endorelations $G \longrightarrow G$, we talk about bisimilarity in $G$.

Notation 2.10. Bisimilarity in $G$ over $A$ is denoted by $\sim_{A}$. It may, upon a slight abuse of notation, be understood as an equivalence relation over all vertices of any two graphs over $A$. Namely, if $G$ and $G^{\prime}$ are graphs over $A$, we may write $x \sim_{A} y$ when $x \in G$ and $y \in G^{\prime}$ to mean bisimilarity in $G+G^{\prime}$.

Before treating weak bisimulations, we consider a first stability result, which is all we need about strong bisimulations.

Any morphism $f: A \rightarrow B$ induces by pullback a change-of-base functor $\Delta_{f}: \mathrm{Gph} / B \rightarrow$ $\mathrm{Gph} / A$, which has a left adjoint $\Sigma_{f}$ given by composition with $f$.

Proposition 2.11. For any morphism of graphs $f: A \rightarrow B$, both functors $\Delta_{f}: \mathrm{Gph} / B \rightarrow$ $\mathrm{Gph} / A$ and $\Sigma_{f}: \mathrm{Gph} / A \rightarrow \mathrm{Gph} / B$, i.e., pullback along and post-composition with $f$, preserve functional bisimulations.

Proof. The case of $\Sigma_{f}$ is actually trivial. For $\Delta_{f}$, we use Remark 2.8, By the pullback lemma, the square on the right below is a pullback. We check that $\Delta_{f}(G) \rightarrow \Delta_{f}\left(G^{\prime}\right)$ is again a bisimulation. Indeed, consider any square as on the left below:

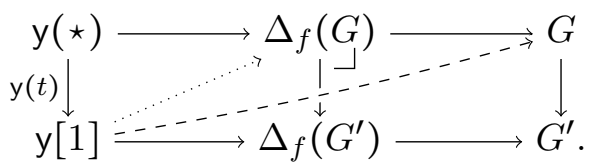

Because $G \rightarrow G^{\prime}$ is a bisimulation, we obtain the dashed arrow making both triangles commute. But then by universal property of pullback, we obtain the dotted arrow, making the corresponding bottom triangle commute. Finally, the top triangle commutes upon postcomposition with $\Delta_{f}(G) \rightarrow G$, and after composition with $\Delta_{f}(G) \rightarrow \Delta_{f}\left(G^{\prime}\right)$, hence commutes by uniqueness in the universal property of pullback.

Remark 2.12. This is an instance of the fact that right maps are stable under pullback in any weak factorisation system [28], here with the factorisation system cofibrantly generated by the sole map y $(t)$.

Let us now treat weak bisimulations. We start with the functional case.

Definition 2.13. A morphism $f: G \rightarrow G^{\prime}$ in $G$ ph/A is a functional, weak bisimulation iff $\mathrm{fc}(f): \mathrm{fc}(G) \rightarrow \mathrm{fc}\left(G^{\prime}\right)$ is a graph fibration.

Proposition 2.14. This equivalent to the fact that, for any edge e: $y^{\prime} \rightarrow f(x)$ in $G^{\prime}$, there exists $x^{\prime}$ in $G$ and a path $r: x^{\prime} \rightarrow^{\star} x$ such that $\overline{f^{\star}(r)}=\widetilde{(e)}$.

Proof. If $e$ is an identity, then taking the empty path for $r$ will do, so the condition really says something about non-identity edges $e$. 
Remark 2.15. Remark 2.8 adapts to weak, functional bisimulations, using $\mathrm{fc}(f)$ instead of $f$.

Let us now handle the relational case. In the strong case, a relation between graphs $G$ and $G^{\prime}$ over $A$ was defined to be a subobject of the pullback $G \times{ }_{A} G^{\prime}$, and simulation properties were related to the projections being graph fibrations. In order to follow this pattern here, we need to consider $\mathrm{fc}(A)$ instead of $A$. However, in general, $\mathrm{fc}(G) \times{ }_{\mathrm{fc}(A)} \mathrm{fc}\left(G^{\prime}\right)$ differs from $\mathrm{fc}\left(G \times{ }_{A} G^{\prime}\right)$. We consider the former:

Definition 2.16. A weak simulation $G \longrightarrow G^{\prime}$ is a relation $R \subseteq \mathrm{fc}(G) \times_{\mathrm{fc}(A)} \mathrm{fc}\left(G^{\prime}\right)$ whose first projection $R \hookrightarrow \mathrm{fc}(G) \times{ }_{\mathrm{fc}(A)} \mathrm{fc}\left(G^{\prime}\right) \rightarrow \mathrm{fc}(G)$ is a graph fibration.

$R$ is a weak bisimulation iff both projections are graph fibrations.

Explicitly, consider $p: G \rightarrow A$ and $p^{\prime}: G^{\prime} \rightarrow A$, and $R$ as above a weak simulation. For any edge $r: x \leftarrow x^{\prime}$ in $\mathrm{fc}(G)$, i.e., identity-free path $r: x \leftarrow{ }^{\star} x^{\prime}$, and $y \in G^{\prime}$ such that $R(x, y)$, there should be an identity-free path $r^{\prime}: y \leftarrow^{\star} y^{\prime}$ in $G^{\prime}$ such that $\left(r, r^{\prime}\right) \in R$. If $R$ is full, this is equivalent to the existence, for each edge $e: x \leftarrow x^{\prime}$ in $G$ and $y \in G^{\prime}$ such that $R(x, y)$, of an identity-free path $r^{\prime}: y \leftarrow^{\star} y^{\prime}$ such that $R\left(x^{\prime}, y^{\prime}\right)$ and $\overline{(p(e))}=\left(\overline{\left.p^{\prime}\right)^{\star}\left(r^{\prime}\right)}\right.$. We will only consider full relations in this paper, hence only the last characterisation will matter to us.

As in the strong case, we have for any fixed $G$ and $G^{\prime}$ over $A$ :

Proposition 2.17. Weak bisimulations are closed under union, and the union of all weak bisimulations, called weak bisimilarity, is again a weak bisimulation, the maximum one.

Notation 2.18. Weak bisimilarity over $A$ is denoted by $\approx_{A}$. As for strong bisimilarity, we will abuse notation and consider $\approx A$ as a relation between the vertices of any two graphs over $A$.

2.3. CCS. The main subject of this paper is CCS [44], and fair testing equivalence over it. We work with a standard version, except in two respects. First, we work with infinite terms, which spares us the need for replication, recursion, or other possible mechanisms for describing infinite processes in a finite way. Second, we work with a de Bruijn-like presentation: terms carry their (finite) sets of known channels, in the form of a finite number. I.e., the number $n$ indicates that the considered process knows channels $1, \ldots, n$ (which complies with our notation for finite ordinals, introduced in Section 2.1).

Remark 2.19. While the de Bruijn-like presentation clearly is a matter of convenience, working with infinite terms does have an impact on our results. Restricting ourselves to recursive processes (e.g., by introducing some recursion construct), we would still have that $(P) \sim_{f}(Q)$ implies $P \sim_{f, s} Q$. The converse is less obvious and may be stated in very simple terms: suppose you have two recursive CCS processes $P$ and $Q$ and a test process $T$, possibly non-recursive, distinguishing $P$ from $Q$; is there any recursive $T^{\prime}$ also distinguishing $P$ from $Q$ ? We leave this question open.

Our (infinite) CCS terms are coinductively generated by the typed grammar

$$
\frac{\Gamma \vdash P \quad \Gamma \vdash Q}{\Gamma \vdash P \mid Q} \quad \frac{\Gamma, a \vdash P}{\Gamma \vdash \nu a . P} \quad \frac{\ldots \quad \Gamma \vdash P_{i} \quad \cdots}{\Gamma \vdash \sum_{i \in n} \alpha_{i} \cdot P_{i}}(n \in \mathbb{N}) .
$$

Here, as announced, $\Gamma$ ranges over $\mathbb{N}$, i.e., the free names of a process always are $1 \ldots n$ for some $n$. Accordingly, $\Gamma, a$ denotes just $n+1$ (and then $a=n+1$ ). Furthermore, $\alpha_{i}$ is 


$$
\begin{array}{cc}
\overline{(\Gamma \vdash P) \stackrel{i d}{\longleftarrow}(\Gamma \vdash P)} & \overline{\left(\Gamma \vdash \sum_{i \in n} \alpha_{i} . P_{i}\right) \stackrel{\alpha_{i}}{\longleftarrow}\left(\Gamma \vdash P_{i}\right)} \\
\frac{\left(\Gamma \vdash P_{1}\right) \stackrel{\alpha}{\leftarrow}\left(\Gamma \vdash P_{1}^{\prime}\right)}{\left(\Gamma \vdash P_{1} \mid P_{2}\right) \stackrel{\alpha}{\leftarrow}\left(\Gamma \vdash P_{1}^{\prime} \mid P_{2}\right)} & \frac{\left(\Gamma \vdash P_{2}\right) \stackrel{\alpha}{\leftarrow}\left(\Gamma \vdash P_{2}^{\prime}\right)}{\left(\Gamma \vdash P_{1} \mid P_{2}\right) \stackrel{\alpha}{\leftarrow}\left(\Gamma \vdash P_{1} \mid P_{2}^{\prime}\right)} \\
\frac{(\Gamma, a \vdash P) \stackrel{\alpha}{\leftarrow}\left(\Gamma, a \vdash P^{\prime}\right)}{(\Gamma \vdash \nu a . P) \stackrel{\alpha}{\leftarrow}\left(\Gamma \vdash \nu a . P^{\prime}\right)}(\alpha \notin\{a, \bar{a}\}) & \frac{\left(\Gamma \vdash P_{1}\right) \stackrel{\alpha}{\leftarrow}\left(\Gamma \vdash P_{1}^{\prime}\right) \quad\left(\Gamma \vdash P_{2}\right) \stackrel{\bar{\alpha}}{\leftarrow}\left(\Gamma \vdash P_{2}^{\prime}\right)}{\left(\Gamma \vdash P_{1} \mid P_{2}\right) \stackrel{i d}{\leftarrow}\left(\Gamma \vdash P_{1} \mid P_{2}^{\prime}\right)}
\end{array}
$$

Figure 1: CCS transitions

either $a, \bar{a}$, or $\odot$ (for $a \in \Gamma$ ). The latter is a 'tick' move used in the definition of fair testing equivalence.

Definition 2.20. Let $\mathbb{A}$ be the reflexive graph with vertices given by finite ordinals, edges $\Gamma \rightarrow \Gamma^{\prime}$ given by $\varnothing$ if $\Gamma \neq \Gamma^{\prime}$, and by $\Gamma+\Gamma+\{i d, \odot\}$ otherwise, $i d: \Gamma \rightarrow \Gamma$ being the identity edge on $\Gamma$. Elements of the first summand are denoted by $a \in \Gamma$, while elements of the second summand are denoted by $\bar{a}$.

We view terms as a graph $C C S$ over $\mathbb{A}$ with the usual transition rules, as recalled in Figure 1 (which is an inductive definition). There, we let $\bar{\alpha}$ denote $\bar{a}$ when $\alpha=a$, or $a$ when $\alpha=\bar{a}$.

Remark 2.21. The graph $\mathbb{A}$ only has 'endo'-edges, hence only relates terms with the same set of free channels. Some LTSs below do use more general graphs.

Let us finally recall the definition of fair testing equivalence. Let $\perp$ denote the set of processes $P$ such that for all paths $P_{\mathbb{A}} \Longleftarrow P^{\prime}$, there exists a path $P^{\prime} \stackrel{\stackrel{\odot}{\rightleftharpoons}}{=} P^{\prime \prime}$.

Definition 2.22. A test for $\Gamma \vdash P$ is any process $\Gamma \vdash Q$. A test $Q$ is passed by $P$ when $(\Gamma \vdash P \mid Q) \in \perp$. Two processes $\Gamma \vdash P$ and $\Gamma^{\prime} \vdash P^{\prime}$ are fair testing equivalent, notation $(\Gamma \vdash P) \sim_{f, s}\left(\Gamma^{\prime} \vdash P^{\prime}\right)$, iff $\Gamma=\Gamma^{\prime}$ and $P$ and $P^{\prime}$ pass exactly the same tests.

\section{Summary of PREvious WORK}

In this section, we recall some material from $\mathrm{HP}$. Apart from the admittedly numerous prerequisites mentioned in the previous section, the paper should be self-contained, although the material in this section would usefully be complemented by reading $\mathrm{HP}$.

As sketched in the introduction, we construct a multi-player game, consisting of positions and plays between them. Positions are certain graph-like objects, where vertices represent players and channels. But what might be surprising is that moves are not just a binary relation between positions, because we not only want to say when there is a move from one position to another, but also how one moves from one to the other. This will be implemented by viewing moves from $X$ to $Y$ as cospans $Y \stackrel{s}{\rightarrow} M \stackrel{t}{\leftarrow} X$ in a certain category $\widehat{\mathbb{C}}^{f}$ of higher-dimensional graph-like objects, or 'string diagrams', where $X$ and $Y$ respectively are the initial and final positions, and $M$ describes how one goes from $X$ 


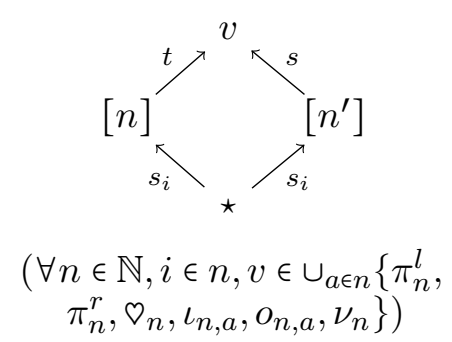

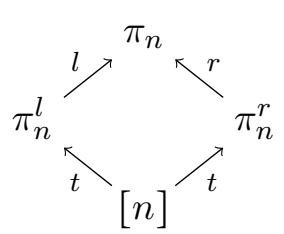

$(\forall n)$

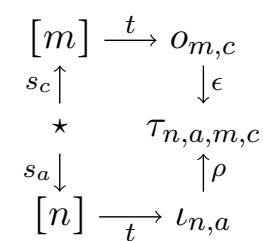

$(\forall n \in \mathbb{N}, a \in n$, and $c \in m)$

Figure 2: Equations for $\mathbb{C}$

to $Y$. By composing such moves (by pushout), we get a bicategory $\mathbb{D}_{v}^{C C S}$ of positions and plays. This is described in Sections 3.1 3.3. In Section 4, we will equip this bicategory with more structure, namely that of a pseudo double category, where one direction models dynamics, and the other models space, e.g., the inclusion of a position into another. Section 3.4 further recalls our two notions of strategies derived from the game (behaviours and innocent strategies, respectively), and Section 3.5 recalls our semantic variant of fair testing equivalence.

3.1. Diagrams. In preparation for the definition of our base category $\mathbb{C}$, recall that (directed, multi) graphs may be seen as presheaves over the category freely generated by the graph with two objects $\star$ and [1], and two edges $s, t: \star \rightarrow$ [1]. Any presheaf $G$ represents the graph with vertices in $G(\star)$ and edges in $G[1]$, the source and target of any $e \in G[1]$ being respectively $e \cdot s$ and $e \cdot t$. A way to visualise how such presheaves represent graphs is to compute their categories of elements [39]. Recall that the category of elements $\int G$ for a presheaf $G$ over $\mathbb{C}$ has as objects pairs $(c, x)$ with $c \in \mathbb{C}$ and $x \in G(c)$, and as morphisms $(c, x) \rightarrow(d, y)$ all morphisms $f: c \rightarrow d$ in $\mathbb{C}$ such that $y \cdot f=x$. This category admits a canonical projection functor $\pi_{G}$ to $\mathbb{C}$, and $G$ is the colimit of the composite $\int G \stackrel{\pi_{G}}{\rightarrow} \mathbb{C} \stackrel{\text { y }}{\rightarrow} \widehat{\mathbb{C}}$ with the Yoneda embedding. E.g., the category of elements for y[1] is the poset $(\star, s) \stackrel{s}{\rightarrow}\left([1], i d_{[1]}\right) \stackrel{t}{\leftarrow}(\star, t)$, which could be pictured as $\longrightarrow$, where dots represent vertices, the triangle represents the edge, and links materialise the graph of $G(s)$ and $G(t)$, the convention being that $t$ goes from the apex of the triangle. We thus recover some graphical intuition.

Our string diagrams will also be defined as (finite) presheaves over some base category $\mathbb{C}$. Let us give the formal definition of $\mathbb{C}$ for reference. We advise to skip it on first reading, as we then attempt to provide some graphical intuition.

Definition 3.1. Let $G_{\mathbb{C}}$ be the graph with, for all $n, m \in \mathbb{N}, a \in n$, and $c \in m$ :

- vertices $\star,[n], \pi_{n}^{l}, \pi_{n}^{r}, \pi_{n}, \nu_{n}, \diamond_{n}, \iota_{n, a}, o_{n, a}$, and $\tau_{n, a, m, c}$;

- edges $s_{1}, \ldots, s_{n}: \star \rightarrow[n]$

- for all $v \in\left\{\pi_{n}^{l}, \pi_{n}^{r}, \diamond_{n}, \iota_{n, a}, o_{n, a}\right\}$, edges $s, t:[n] \rightarrow v$;

- edges $[n] \stackrel{t}{\rightarrow} \nu_{n} \stackrel{s}{\leftarrow}[n+1]$;

- edges $\pi_{n}^{l} \stackrel{l}{\rightarrow} \pi_{n} \stackrel{r}{\leftarrow} \pi_{n}^{r}$;

- edges $\iota_{n, a} \stackrel{\rho}{\rightarrow} \tau_{n, a, m, c} \stackrel{\epsilon}{\leftarrow} o_{m, c}$.

Let $\mathbb{C}$ be the free category on $G_{\mathbb{C}}$, modulo the equations in Figure 2, where, in the left-hand one, $n^{\prime}$ is $n+1$ when $v=\nu_{n}$, and $n$ otherwise. 

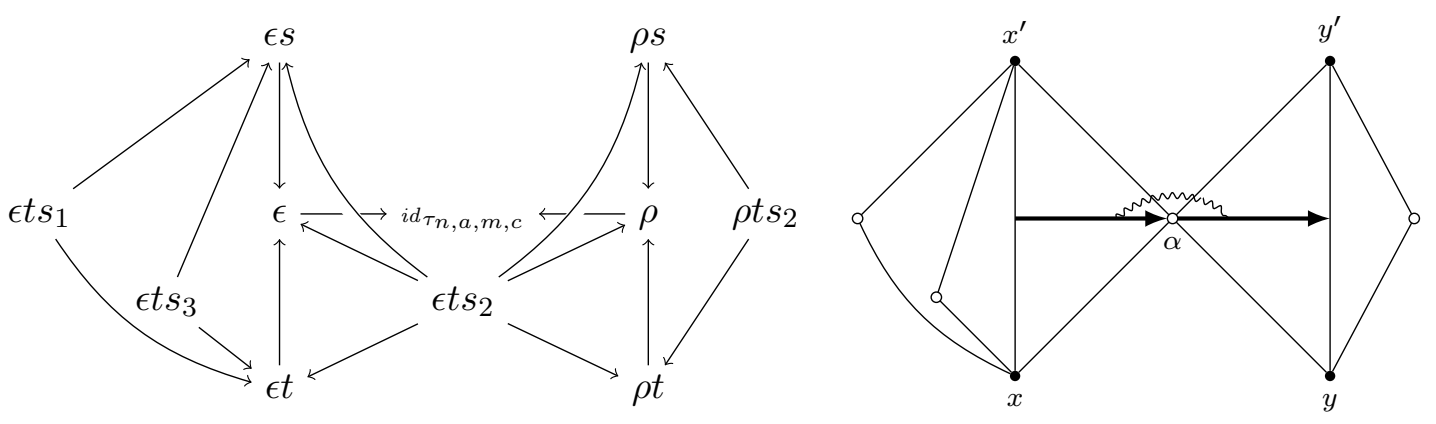

Figure 3: Category of elements for $\tau_{2,1,3,2}$ and graphical representation

Our category of string diagrams will be the category $\widehat{\mathbb{C}}^{f}$ of finite presheaves on $\mathbb{C}$.

To explain this seemingly arbitrary definition, let us compute a few categories of elements. Let us start with an easy one, that of $[3] \in \mathbb{C}$ (we implicitly identify any $c \in \mathbb{C}$ with yc). An easy computation shows that it is the poset pictured in the top part on the right. We will think of it as a position with one player $\left([3], i d_{[3]}\right)$ connected to three channels, and draw it as in the bottom part on the right, where the bullet represents the player, and circles represent channels. The positions

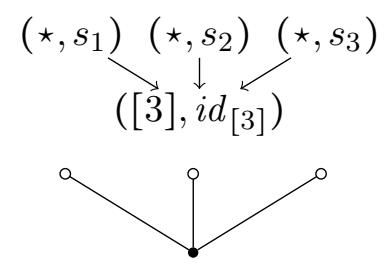
of our game are finite presheaves empty except perhaps on $\star$ and [ $n]$ 's. Other objects will represent moves. The graphical representation is slightly ambiguous, because the ordering of channels known to players is implicit. We will disambiguate in the text when necessary. A morphism of positions is an injective morphism of presheaves. The intuition for a morphism $X \rightarrow Y$ between positions is thus that $X$ embeds into $Y$.

Definition 3.2. Positions and morphisms between them form a category $\mathbb{D}_{h}^{C C S}$.

A more difficult category of elements is that of $\pi_{2}$. It is the poset generated by the graph on the left (omitting base objects for conciseness):
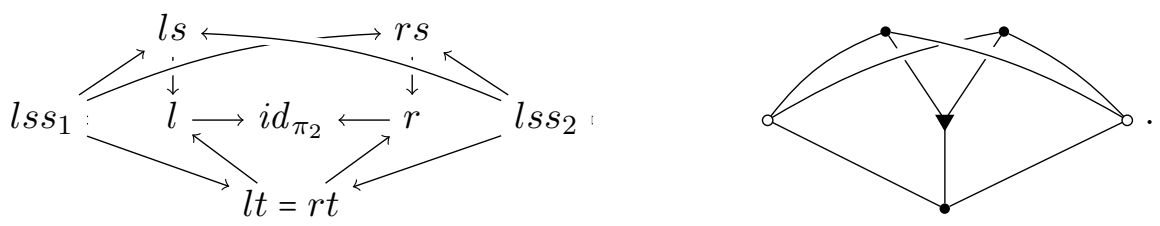

We think of it as a binary player (lt) forking into two players ( $l s$ and $r s$ ), and draw it as on the right. The graphical convention is that a black triangle stands for the presence of $i d_{\pi_{2}}, l$, and $r$. Below, we represent just $l$ as a white triangle with only a left-hand branch, and symmetrically for $r$. Furthermore, in all our pictures, time flows 'upwards'.

Another category of elements, characteristic of CCS, is the one for synchronisation $\tau_{n, a, m, c}$. The case $(n, a, m, c)=(2,1,3,2)$ is the poset generated by the graph on the left of Figure 3, which we will draw as on the right. The left-hand ternary player $x$ outputs on its 2nd channel, here $\alpha$. The right-hand unary player $y$ receives on its 1st channel, again $\alpha$. Both players have two occurrences, one before and one after the move, respectively marked as $x / x^{\prime}$ and $y / y^{\prime}$. Both $x$ and $x^{\prime}$ have arity 3 here, and both $y$ and $y^{\prime}$ have arity 1 . There 


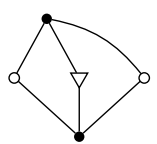

$[n]$

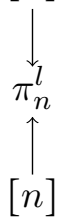

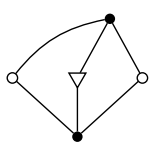

$[n]$

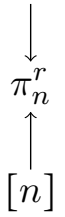

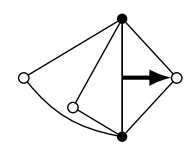

$[m]$

$\downarrow$

$o_{m, c}$

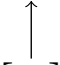

$[m]$

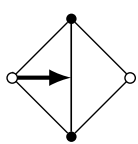

$[n]$

$\downarrow$

$\iota_{n, a}$

$\uparrow$

$[n]$

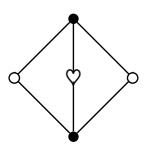

$[n]$

$\downarrow$

$\nabla_{n}$

$\uparrow$
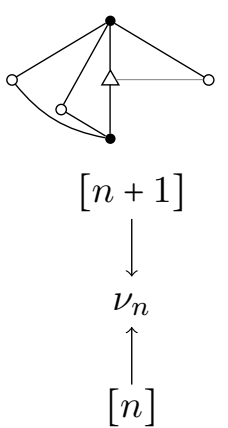

Figure 4: String diagrams and corresponding cospans for $\pi_{n}^{l}, \pi_{n}^{r}, o_{m, c}, \iota_{n, a}, \odot_{n}$, and $\nu_{n}$

are actually three moves, in the sense that there are three higher-dimensional objects in the corresponding category of elements. The first is the output move from $x$ to $x^{\prime}$, graphically represented as the left-hand $\rightarrow$ (intended to evoke the 'ping' sent by $x$ entering channel $\alpha$ ). The second move is the input move from $y$ to $y^{\prime}$, graphically represented as the righthand $\longrightarrow$ (intended to evoke a 'ping' exiting channel $\alpha$ ). The third and final move is the synchronisation itself, which 'glues' the other two together, as represented by the squiggly line.

We leave the computation of other categories of elements as an exercise to the reader. The remaining diagrams are depicted in the top row of Figure 4 , for $(n, a, m, c)=(2,1,3,2)$. The first two are views, in the game semantical sense, of the fork move $\pi_{2}$ explained above. The next two, $o_{m, c}$ (for 'output') and $\iota_{n, a}$ (for 'input'), respectively represent what the sender and receiver can see of the above synchronisation move. The last two diagrams are a 'tick' move, used for defining fair testing equivalence, and a channel creation move.

3.2. From diagrams to moves. In the previous section, we have defined our category of diagrams as $\widehat{\mathbb{C}}^{f}$, and provided some graphical intuition on its objects. The next goal is to construct a bicategory whose objects are positions (recall: presheaves empty except perhaps on $\star$ and $[n]$ 's), and whose morphisms represent plays in our game. We start in this section by defining moves as cospans in $\widehat{\mathbb{C}}^{f}$, and continue in the next one by explaining how to compose moves to form plays. Moves are defined in two stages: seeds, first, give the local form for moves, which are then defined by embedding seeds into bigger positions.

To start with, until now, our diagrams contain no information about the 'flow of time' (although it was mentioned informally for pedagogical purposes). To add this information, for each diagram $M$ representing a move, we define its initial and final positions, say $X$ and $Y$, and view the whole move as a cospan $Y \stackrel{s}{\rightarrow} M \stackrel{t}{\leftarrow} X$. We have taken care, in drawing our diagrams before, of placing initial positions at the bottom, and final positions at the top. We leave it to the reader to define, based on the above pictures, the cospans
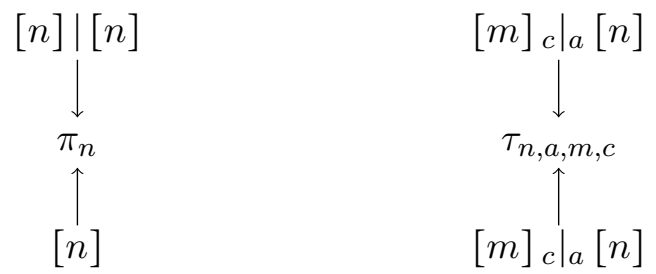
for forking and synchronisation, plus the ones specified in the bottom row of Figure 4. In these cospans, initial positions are on the bottom row, and we denote by $\left.[m]_{c_{1}, \ldots, c_{p}}\right|_{a_{1}, \ldots, a_{p}}[n]$ the position consisting of an $m$-ary player $x$ and an $n$-ary player $y$, quotiented by the equations $x \cdot s_{c_{k}}=y \cdot s_{a_{k}}$ for all $k \in p$. When both lists are empty, by convention, $m=n$ and the players share all channels in order.

Definition 3.3. These cospans are called seeds.

Remark 3.4. Such cospans will be used below as the morphisms of a bicategory $\mathbb{D}_{v}^{C C S}$, using their lower object as their target. Thus, we often denote the corresponding leg by $t$ and the other by $s$. The reason for this convention is that it emphasises below that the fibration axiom (P1) is very close to a universal property of pullback [27].

Remark 3.5. Both legs of each seed are monic, as will be below both legs of each move, and then of each play (because monics are stable under pushout in presheaf categories).

As announced, the moves of our game are obtained by embedding seeds into bigger positions. This means, e.g., allowing a fork move to occur in a position with more than one player. We proceed as follows.

Definition 3.6. Let the interface of a seed $Y \stackrel{s}{\rightarrow} M \stackrel{t}{\leftarrow} X$ be $I_{X}=X(\star) \cdot \star$, i.e., the position consisting only of the channels of the initial position of the seed. More generally, an interface is a position consisting only of channels.

Since channels present in the initial position remain in the final one, we have for each seed a commuting diagram as on the right. By gluing any position $Z$ to the seed along its interface, we obtain a new

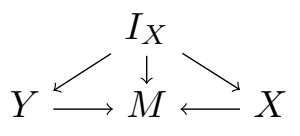
cospan, say $Y^{\prime} \rightarrow M^{\prime} \leftarrow X^{\prime}$. I.e., for any injective morphism $I_{X} \rightarrow Z$, we push $I_{X} \rightarrow X$, $I_{X} \rightarrow M$, and $I_{X} \rightarrow Y$ along $I_{X} \rightarrow Z$ and use the universal property of pushout, as in:

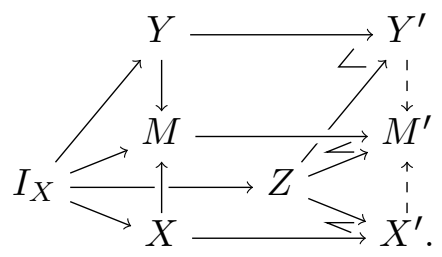

Definition 3.7. Let moves be all cospans obtained in this way.

Recall that colimits in presheaf categories are pointwise. So, e.g., taking pushouts along injective maps graphically corresponds to gluing diagrams together.

Example 3.8. The cospan [2] $\mid[2] \stackrel{[l s, r s]}{\longrightarrow} \pi_{2} \stackrel{l t}{\leftarrow}[2]$ has as canonical interface the presheaf $I_{[2]}=2 \cdot \star$, consisting of two channels, say $a$ and $b$. Consider the position [2] $+\star$ consisting of a player $y$ with two channels $b^{\prime}$ and $c$, plus an additional channel $a^{\prime}$. Further consider the map $h: I_{[2]} \rightarrow[2]+\star$ defined by $a \mapsto a^{\prime}$ and $b \mapsto b^{\prime}$. The pushout

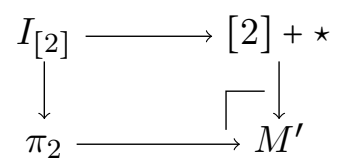

is

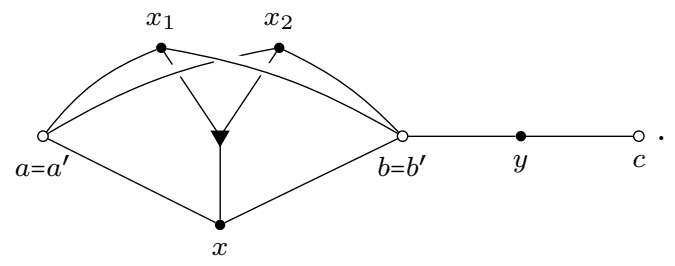


We conclude with a useful classification of moves.

Definition 3.9. A move is full iff it is neither a left nor a right fork. A seed is basic iff it is neither a full fork nor a synchronisation. We call $\mathbb{F}$ the identity-on-objects subgraph of Cospan $\left(\widehat{\mathbb{C}}^{f}\right)$ spanning full moves.

Intuitively, a move is full when its final position contains all possible avatars of involved players.

3.3. From moves to plays. Having defined moves, we now define their composition to construct our bicategory $\mathbb{D}_{v}^{C C S}$ of positions and plays. $\mathbb{D}_{v}^{C C S}$ will be a sub-bicategory of Cospan $\left(\widehat{\mathbb{C}}^{f}\right)$, the bicategory which has as objects all finite presheaves on $\mathbb{C}$, as morphisms $X \rightarrow Y$ all cospans $X \rightarrow U \leftarrow Y$, and as 2-cells $U \rightarrow V$ all com-

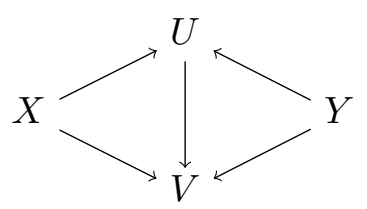
muting diagrams as on the right. Composition is given by pushout, and hence not strictly associative.

Definition 3.10. Let $\mathbb{D}_{v}^{C C S}$ denote the locally full subbicategory of Cospan $\left(\widehat{\mathbb{C}}^{f}\right)$ with positions as objects, whose morphisms, plays, are either equivalences or isomorphic to some composite of moves.

We denote morphisms in Cospan $\left(\widehat{\mathbb{C}}^{f}\right)$ with special arrows $X \rightarrow Y$; composition and identities are denoted with $\bullet$ and $i d^{\bullet}$ (recalling the notation for vertical morphisms in a pseudo double category in Section 2.11).

Again, composition by pushout glues diagrams on top of each other.

Example 3.11. Composition features some concurrency. Composing the move of Example 3.8 with a forking move by $y$ yields

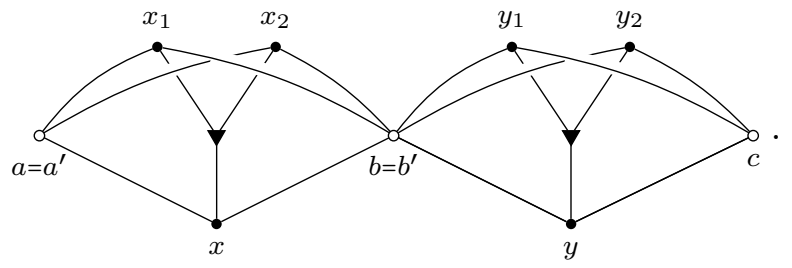

Example 3.12. Composition retains causal dependencies between moves. To see this, consider the following diagram. In the initial position, there are channels $a$ and $b$, plus three players $x(b), y(a, b)$, and $z(a)$ (we indicate the channels known to each player in parentheses). In a first move, $x$ outputs on $b$, while $y$ inputs. In a second move, $z$ outputs on $a$, while (the avatar $y^{\prime}$ of) $y$ inputs. The fact that $y$ first inputs on $b$ then on $a$ is encoded in the corresponding diagram, which looks like the following:

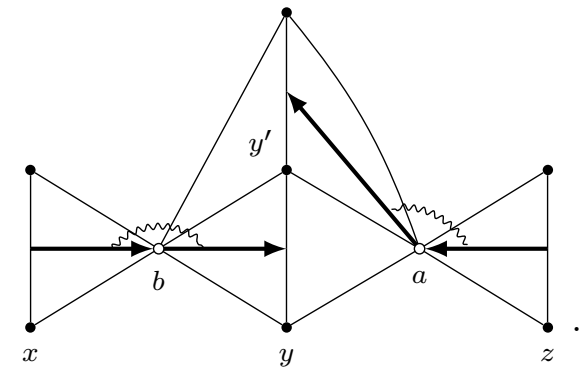




\subsection{Behaviours and strategies.}

3.4.1. Behaviours. Recall from $\mathrm{HP}$ the category $\mathbb{E}$

- whose objects are maps $U \leftarrow X$ in $\widehat{\mathbb{C}}^{f}$, such that there exists a play $Y \rightarrow U \leftarrow X$, i.e., objects are plays, where we forget the final position;

- and whose morphisms $(U \leftarrow X) \rightarrow\left(U^{\prime} \leftarrow X^{\prime}\right)$ are commuting diagrams as on the right with all arrows monic.

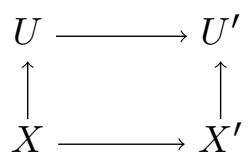

Morphisms $(U \leftarrow X) \rightarrow\left(U^{\prime} \leftarrow X^{\prime}\right)$ in $\mathbb{E}$ represent extensions of $U$, both spatially (i.e., embedding into a larger position) and dynamically (i.e., adding more moves).

We may relativise this category $\mathbb{E}$ to a particular position $X$, yielding a category $\mathbb{E}(X)$ of plays on $X$ as follows. Consider the functor cod: $\mathbb{E} \rightarrow \mathbb{D}_{h}^{C C S}$ mapping any play $U \leftarrow X$ to its initial position $X$, and consider the pullback category $\mathbb{E}(X)$ as defined in Section 2.1. The objects of $\mathbb{E}(X)$ are just plays $(U \leftarrow X)$ on $X$, and morphisms are morphisms of plays whose lower border is $i d_{X}$. This yields the definition of a category of 'naive' strategies, called behaviours.

Definition 3.13. The category $\mathrm{B}_{X}$ of behaviours on $X$ is the category $\overline{\mathbb{E}(X)}$ of presheaves of finite sets on $\mathbb{E}(X)$.

Behaviours suffer from the deficiency of allowing unwanted cooperation between players.

Example 3.14. Consider a position $X$ with three players $x, y, z$ sharing a channel $a$, and the following plays on it: in $u_{x, y}, x$ outputs on $a$, and $y$ inputs; in $u_{x, z}, x$ outputs on $a$, and $z$ inputs; in $i_{z}, z$ inputs on $a$. One may define a behaviour $S$ mapping $u_{x, y}$ and $i_{z}$ to a singleton, and $u_{x, z}$ to $\varnothing$. Because $u_{x, y}$ is accepted, $x$ accepts to output on $a$; and because $i_{z}$ is accepted, $z$ accepts to input on $a$. The problem is that $S$ rejecting $u_{x, z}$ roughly amounts to $x$ refusing to synchronise with $z$, or conversely.

\subsubsection{Strategies. To rectify this, we consider the following notion of view:}

Definition 3.15. Let $\mathbb{E}^{\mathbb{V}}$ denote the full subcategory of $\mathbb{E}$ consisting of views, i.e., composites of basic seeds.

We relativise views to a position $X$ by considering the comma category $\mathbb{E}_{X}^{\mathbb{V}}$ as defined in Section 2.1, Its objects are pairs of a view $V \leftarrow[n]$ on a single $n$-ary player, and an embedding $[n] \hookrightarrow X$, i.e., a player of $X$.

Definition 3.16. The category $S S_{X}$ of strategies on $X$ is the category $\widetilde{\mathbb{E}_{X}^{\mathbb{V}}}$ of presheaves of finite ordinals on $\mathbb{E}_{X}^{\mathbb{V}}$.

Remark 3.17. We could here replace finite ordinals with a wider category and still get a valid semantics. But then to show the correspondence with the syntax below we would work with the subcategory of presheaves of finite ordinals.

This definition of strategies rules out undesired behaviours. We now sketch how to map strategies to behaviours (this is done in more detail for arbitrary playgrounds below): let first $\mathbb{E}_{X}$ be the category obtained by taking a comma category instead of a pullback in the definition of $\mathbb{E}(X)$. Then, embedding $\widehat{\mathbb{E}_{X}^{\mathbb{V}}}$ into $\overline{\mathbb{E}_{X}^{\mathbb{V}}}$ via ford $\hookrightarrow$ set, followed by right Kan extension to $\mathbb{E}_{X}^{o p}$ followed by restriction to $\mathbb{E}(X)^{o p}$ yields a functor $\overline{(-)}: S S_{X} \rightarrow \mathrm{B}_{X}$. The image of a strategy $S$ may be computed as in 


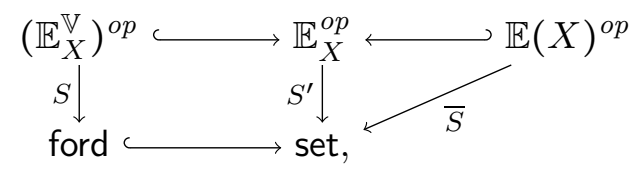

where $S^{\prime}$ is here obtained by right Kan extension (the embedding $\left(\mathbb{E}_{X}^{\mathbb{V}}\right)^{o p} \hookrightarrow \mathbb{E}_{X}^{o p}$ being full and faithful, we may choose the diagram to strictly commute). By the standard formula for right Kan extensions as ends [38] we have, for any $S:\left(\mathbb{E}_{X}^{\mathbb{V}}\right)^{o p} \rightarrow$ ford:

$$
\bar{S}(U)=\int_{v \in \mathbb{E}_{X}^{\mathbb{V}}} S(v)^{\mathbb{E}_{X}(v, U)} .
$$

If $S$ is boolean, i.e., takes values in $\{\varnothing, 1\}$, then the involved end may be viewed as a conjunction, saying that $U$ is accepted by $\bar{S}$ whenever all its views are accepted by $S$. Equivalently, $\bar{S}(U)$ is a limit of $\left(\mathbb{E}_{X}^{\mathbb{V}} / U\right)^{o p} \stackrel{\text { dom }}{\longrightarrow}\left(\mathbb{E}_{X}^{\mathbb{V}}\right)^{o p} \stackrel{S}{\rightarrow}$ ford $\hookrightarrow$ set.

3.4.3. Decomposition: a syntax for strategies. Our definition of strategies is rather semantic in flavour. Indeed, presheaves are akin to domain theory. However, they also lend themselves well to a syntactic description (unlike behaviours). Again, this is treated at length in the abstract setting below, so we here only sketch the construction.

First, it is shown in $\mathrm{HP}$ that strategies on an arbitrary position $X$ are in 1-1 correspondence with families of strategies indexed by the players of $X$. Recall that $[n]$ is the position consisting of one $n$-ary player. A player of $X$ is the same as a morphism $[n] \rightarrow X$ (for some $n)$ in $\mathbb{D}_{h}^{C C S}$. Thus, we define the set $\operatorname{Pl}(X)=\sum_{n \in \mathbb{N}} \mathbb{D}_{h}^{C C S}([n], X)$ of players of $X$.

Proposition 3.18. We have $S S_{X} \cong \prod_{(n, x) \in \operatorname{Pl}(X)} S S_{[n]}$. For any $S \in S S_{X}$, we denote by $S \cdot x$ the component corresponding to $x \in \mathrm{Pl}(X)$ under this isomorphism.

So, strategies on arbitrary positions may be entirely described by strategies on 'typical' players $[n]$. As an important particular case, we may let two strategies interact along an interface (recall from Definition 3.6 that this means a position consisting only of channels), which will be the basis of our semantic definition of fair testing equivalence. We proceed as follows. Consider any pushout $Z$ of $X \leftarrow I \rightarrow Y$ where $I$ is an interface. We have

Corollary 3.19. $S S_{Z} \cong S S_{X} \times S S_{Y}$.

Proof. We have $\mathbb{E}_{Z}^{\mathbb{V}} \cong \mathbb{E}_{X}^{\mathbb{V}}+\mathbb{E}_{Y}^{\mathbb{V}}$, and conclude by universal property of coproduct.

We denote by $[S, T]$ the image of $(S, T) \in S S_{X} \times S S_{Y}$ under this isomorphism.

Having shown how strategies may be decomposed into strategies on 'typical' players $[n]$, we now explain that strategies on such players may be further decomposed. First, we observe that $\mathbb{E}_{[n]}^{\mathbb{V}}$ is isomorphic to the full subcategory $\mathbb{E}^{\mathbb{V}}([n])$ of $\mathbb{E}([n])$ spanning views. For any strategy $S$ on $[n]$ and seed $b:\left[n^{\prime}\right] \rightarrow[n]$, let the residual $S \cdot b$ of $S$ after $b$ be the strategy playing like $S$ after $b$, i.e., for all $v \in \mathbb{E}_{\left[n^{\prime}\right]}^{\mathbb{V}},(S \cdot b)(v)=S(b \bullet v)$. S is almost determined by its residuals. The only information missing from the $S \cdot b$ 's to reconstruct $S$ is the set of initial states and how they relate to the initial states of each $(S \cdot b)$. This may be taken into account as follows.

Definition 3.20. For any $S \in S S_{[n]}$ and initial state $\sigma \in S\left(i d^{\bullet}\right)$, let $S_{\mid \sigma}$, the restriction of $S$ to $\sigma$, be determined by

$$
S_{\mid \sigma}(v)=\left\{\sigma^{\prime} \in S(v) \mid S\left(!_{v}\right)\left(\sigma^{\prime}\right)=\sigma\right\},
$$


where $!_{v}$ denotes the unique morphism !: $i d^{\bullet} \rightarrow v$.

$S$ is determined by its set $S\left(i d^{\bullet}\right)$ of initial states, plus the map $(\sigma, b) \mapsto\left(S_{\mid \sigma} \cdot b\right)$ sending any $\sigma \in S\left(i d^{\bullet}\right)$ and isomorphism class $b$ of seeds to $S_{\mid \sigma} \cdot b$. In other words, we have for all $n$ :

Theorem 3.21. $S S_{[n]} \cong\left(\prod_{n^{\prime} \in \mathbb{N}, b:\left[n^{\prime}\right] \rightarrow[n]} S S_{\left[n^{\prime}\right]}\right)^{\star}$.

Given an element $\left(D_{1}, \ldots, D_{m}\right)$ of the right-hand side, the corresponding strategy maps the identity view $i d^{\bullet}$ to $m$, and any non-identity view $b \bullet v$ on $[n]$ to the sum $\sum_{i \in m} D_{i}(b)(v)$.

A closely related result is that strategies on a player $[n]$ are in bijection with infinite terms in the following typed grammar, with judgements $n$ 衴 $D$ and $n \vdash S$, where $D$ is called a definite strategy and $S$ is a strategy:

$$
\frac{\ldots n_{b} \vdash S_{b} \ldots\left(\forall b:\left[n_{b}\right] \rightarrow[n] \in[\mathbb{B}]_{n}\right)}{n \vdash_{\mathrm{D}}\left\langle\left(S_{b}\right)_{b \in[\mathbb{B}]_{n}}\right\rangle} \quad \frac{\ldots n \vdash_{\mathrm{D}} D_{i} \ldots(\forall i \in m)}{n \vdash \oplus_{i \in m} D_{i}}(m \in \mathbb{N}) .
$$

Here, $[\mathbb{B}]_{n}$ denotes the set of all isomorphism classes of seeds from $[n]$. This achieves the promised syntactic description of strategies. We may readily define the translation of CCS processes, coinductively, as follows. For processes with channels in $\Gamma$, we define

$$
\begin{aligned}
& \left(\sum_{i \in n} \alpha_{i} \cdot P_{i}\right)=\left\langle b \mapsto \oplus_{\left\{i \in n \mid b=\left(\alpha_{i}\right)\right\}}\left(P_{i}\right)\right\rangle \quad(a)=\iota_{\Gamma, a} \\
& (\nu a . P)=\left\langle\nu_{\Gamma} \mapsto(P),_{-} \mapsto \varnothing\right\rangle
\end{aligned}
$$

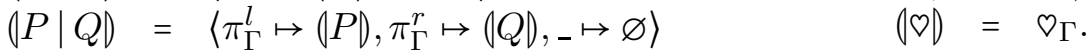

For example, $a \cdot P+a \cdot Q+\bar{b} \cdot R$ is mapped to

$$
\left\langle\iota_{\Gamma, a} \mapsto((P) \oplus|Q|), o_{\Gamma, b} \mapsto(R),,_{-} \mapsto \varnothing\right\rangle .
$$

3.5. Semantic fair testing. The tools developed in the previous section yield the following semantic analogue of fair testing equivalence.

Definition 3.22. Closed-world moves are those generated by some seed among $\nu_{n}, \nabla_{n}, \pi_{n}$, and $\tau_{n, i, m, j}$. A play is closed-world when it is a composite of closed-world moves. Let a closed-world play be successful when it contains a $\odot$ move, and unsuccessful otherwise. A state $\sigma \in B(U)$ of a behaviour $B \in \mathrm{B}_{Z}$ over a closed-world play $U \leftarrow Z$ is successful when the play $U$ is, and unsuccessful otherwise.

Let then $\Perp_{Z}$ denote the set of behaviours $B \in \mathrm{B}_{Z}$ such that any unsuccessful, closedworld state admits a successful extension. Formally:

Definition 3.23. Let $B \in \Perp_{Z}$ iff, for any unsuccessful, closed-world play $U \leftarrow Z$ and $\sigma \in B(U)$, there exists a successful, closed-world $U^{\prime}$, a morphism $f: U \rightarrow U^{\prime}$ in $\mathbb{E}(Z)$, and a state $\sigma^{\prime} \in B\left(U^{\prime}\right)$ such that $\sigma^{\prime} \cdot f=\sigma$.

Finally, let us say that a triple $(I, h, S)$, for any $h: I \rightarrow X$ (where $I$ is an interface) and $S \in S S_{X}$, passes the test consisting of a morphism $k: I \rightarrow Y$ of positions and a strategy $T \in S S_{Y}$ iff $\overline{[S, T]} \in \Perp_{Z}$, where $Z$ is the pushout of $h$ and $k$. Let $(I, h, S)^{\Perp}$ denote the set of all such $(k, T)$.

Definition 3.24. For any $h: I \rightarrow X, h^{\prime}: I^{\prime} \rightarrow X^{\prime}, S \in S S_{X}$, and $S^{\prime} \in S S_{X^{\prime}},(I, h, S) \sim_{f}$ $\left(I^{\prime}, h^{\prime}, S^{\prime}\right)$ iff $I=I^{\prime}$ and $(I, h, S)^{\Perp}=\left(I, h^{\prime}, S^{\prime}\right)^{\Perp}$. 
Obviously, $\sim_{f}$ is an equivalence relation, analogous to standard fair testing equivalence, which we hence also call (semantic) fair testing equivalence.

This raises the question of whether the translation $(-)$ preserves or reflects fair testing equivalence. The rest of the paper is devoted to proving that it does both. As announced in the introduction, this is done by organising the game into a playground, as defined in the next section.

\section{Playgrounds: From Behaviours to Strategies}

4.1. Motivation: a pseudo double category. We start by organising the game described above into a (pseudo) double category. We have seen that positions are the objects of the category $\mathbb{D}_{h}^{C C S}$, whose morphisms are embeddings of positions. We have also seen that positions are the objects of the bicategory $\mathbb{D}_{v}^{C C S}$, whose morphisms are plays. It should seem natural to define a pseudo double

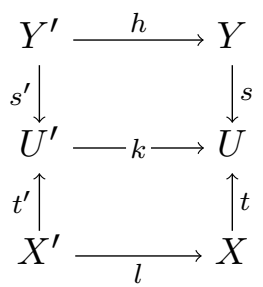
category structure with

- $\mathbb{D}_{h}^{C C S}$ as horizontal category,

- $\mathbb{D}_{v}^{C C S}$ as vertical bicategory,

- commuting diagrams as on the right as double cells.

Here, $X$ is the initial position and $Y$ is the final one; all arrows are mono. This forms a pseudo double category $\mathbb{D}^{C C S}$, and we have:

Proposition 4.1. The functor $\operatorname{cod}_{v}: \mathbb{D}_{H}^{C C S} \rightarrow \mathbb{D}_{h}^{C C S}$ is a Grothendieck fibration [27].

Intuitively, $\operatorname{cod}_{v}$ being a fibration demands some canonical way of restricting a given play on some position $X$ to some 'subposition' $X^{\prime} \rightarrow X$. More technically, it amounts to the existence, for all plays $Y \stackrel{u}{\rightarrow} X$ and horizontal morphisms $X^{\prime} \stackrel{l}{\rightarrow} X$, of a universal $(\approx$ maximal) way of restricting $u$ to $X^{\prime}$, as on the left below:
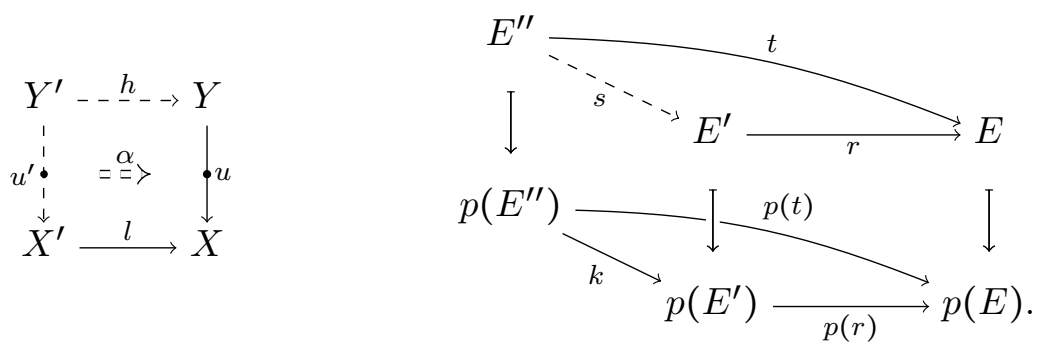

Formally, consider any functor $p: \mathbb{E} \rightarrow \mathbb{B}$. A morphism $r: E^{\prime} \rightarrow E$ in $\mathbb{E}$ is cartesian when, as on the right above, for all $t: E^{\prime \prime} \rightarrow E$ and $k: p\left(E^{\prime \prime}\right) \rightarrow p\left(E^{\prime}\right)$, if $p(r) \circ k=p(t)$ then there exists a unique $s: E^{\prime \prime} \rightarrow E^{\prime}$ such that $p(s)=k$ and $r \circ s=t$.

Definition 4.2. A functor $p: \mathbb{E} \rightarrow \mathbb{B}$ is a fibration iff for all $E \in \mathbb{E}$, any $h: B^{\prime} \rightarrow p(E)$ has a cartesian lifting, i.e., a cartesian antecedent by $p$.

Proposition 4.1 is proved among other facts in Section 7 . This was the starting point of the notion of playground: which axioms should we demand of a pseudo double category in order to enable the constructions of HP? We follow the constructions in this section, considering an arbitrary pseudo double category $\mathbb{D}$, on which we impose axioms along the 
way. Objects and vertical morphisms will respectively be called positions and plays. The pseudo double category $\mathbb{D}^{C C S}$ does satisfy the axioms, albeit in a non-trivial way. This is stated and proved in Section 17, but we use the result in advance in examples to illustrate our constructions.

Let us record the axioms imposed on $\mathbb{D}$ in the next sections to obtain our bisimulation result (Theorem 5.35):

- (P1), page 25,

- (P2) (P5), page 27,

- (P6), page 27.

- (P7), page 28,
- (P8), page 28,

- (P9), page 35.

- (P10) page 42 .

4.2. Behaviours. The easiest construction of $\mathrm{HP}$ to carry over to the abstract setting of playgrounds is that of behaviours. First, let us stress that, in the case of $\mathbb{D}^{C C S}, \mathbb{D}_{H}^{C C S}$ is very different from the category of plays called $\mathbb{E}$ recalled in Section 3.4.1. Indeed, any morphism $\alpha: u \rightarrow u^{\prime}$ in $\mathbb{D}_{H}^{C C S}$ in particular induces an embedding of the final position $\operatorname{dom}(u)$ of $u$ into that of $u^{\prime}$. In $\mathbb{E}$, instead, a morphism $U \rightarrow U^{\prime}$ may involve extending $U$ with more moves.

Example 4.3. The move of Example 3.8 embeds into the play of Example 3.11 in the sense of $\mathbb{E}$, but not in the sense of $\mathbb{D}_{H}^{C C S}$. Indeed, the passive player $y$ of Example 3.8 does belong to the final position, but its image in Example 3.11 does not.

So our first step is to construct an analogue of $\mathbb{E}$ from any playground $\mathbb{D}$. Intuitively, it should have as objects all plays, and as morphisms $u \rightarrow u^{\prime}$ all pairs $(w, \alpha)$ as on the right. However, this definition is slightly wrong on morphisms, in that $\alpha$ carries some information about how $w$ embeds into $u^{\prime}$, while we are only interested in how $u$ does. Thus, we instead

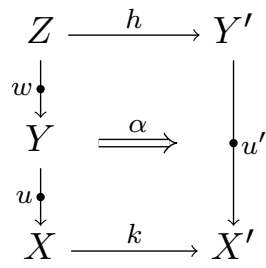
define morphisms $u \rightarrow u^{\prime}$ to be pairs $(w, \alpha)$ as in (4.1), quotiented by the equivalence relation generated by pairs $\left((w, \alpha),\left(w^{\prime}, \beta\right)\right)$ such that there exists morphisms $i$ and $\gamma$ satisfying $\alpha=\beta \circ(u \bullet \gamma)$, as in

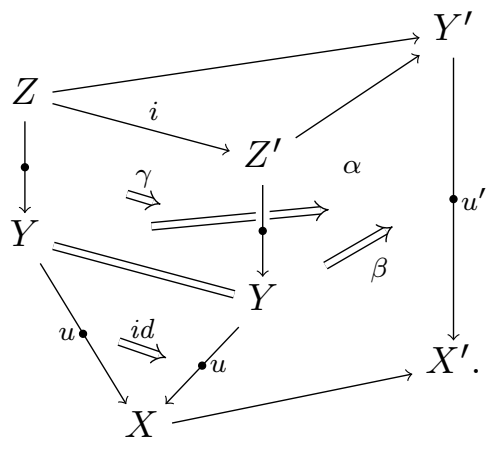

In order to define composition in this category, we state the following axiom (cf. Proposition 4.1).

Axiom. (P1) (Fibration) The vertical codomain functor cod $: D \rightarrow \mathbb{D}_{h}$ is a fibration.

Composition may now be defined by pullback (i.e., cartesian lifting in the fibration $\left.\operatorname{cod}: D \rightarrow \mathbb{D}_{h}\right)$ and pasting: 


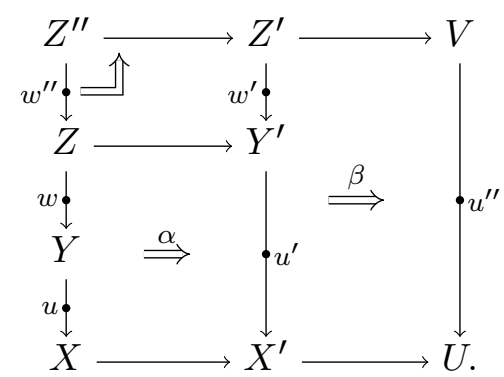

(We use 'double pullback' marks to denote cartesian double cells.) Quotienting makes composition functional and associative, and furthermore it is compatible with the above equivalence. Identities are obvious.

Proposition 4.4. This forms a category $\mathbb{E}$.

Example 4.5. Consider the move $M^{\prime}$ from Example 3.8, and let us name its initial and final positions as in $M^{\prime}: Y^{\prime} \rightarrow X^{\prime}$. Let us further call $U: Y^{\prime \prime} \rightarrow X^{\prime}$ the play from Example 3.11, obtained by composing $M^{\prime}$ with a forking move by $y \in Y^{\prime}[2]$. In order to obtain a double cell $M^{\prime} \rightarrow U$, we need to provide an extension of $M^{\prime}$ with some move by $y$, and there are actually three ways of doing this. One is with a left forking move, another is with a right forking move, and the last is with a full forking move. In this example, the last possibility actually yields an identity double cell $U \rightarrow U$, and may be obtained using (P1) in the following general way. Consider any double cell $\alpha: u \rightarrow u^{\prime}$ in $D$, and play $w^{\prime}$ such that $u^{\prime} \bullet w^{\prime}$ is well-defined. Then, letting $\beta: w \rightarrow w^{\prime}$ be the cartesian lifting of $w^{\prime}$ along $\operatorname{dom}(\alpha)$, we obtain a morphism $u \rightarrow u^{\prime} \bullet w^{\prime}$ in $\mathbb{E}$, as in on the right. The universal property of $\beta$ here amounts to the fact that left and right forking moves both embed uniquely into full forking, which makes our three candidate morphisms $u \rightarrow u^{\prime} \bullet w^{\prime}$ equal

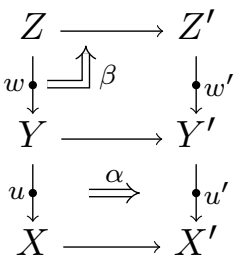
in $\mathbb{E}$.

Recalling notation from Section 2.1, consider now the pullback category $\mathbb{E}(X)$, where $X$ is any position. Following Definition 3.13, we state:

Definition 4.6. The category $\mathrm{B}_{X}$ of behaviours on $X$ is $\overline{\mathbb{E}(X)}$, i.e., the category of presheaves of finite sets on $\mathbb{E}(X)$.

This construction has a bit of structure. Indeed, the map $X \mapsto \mathbb{E}(X)$ extends to a pseudo functor $\mathbb{E}(-): \mathbb{D}_{v} \rightarrow$ Cat by vertical post-composition. Post-composing the opposite of this pseudo functor by $\overline{(-)}$ : Cat ${ }^{o p} \rightarrow$ Cat, we obtain a pseudo functor $\mathrm{B}_{-}: \mathbb{D}_{v}^{o p} \rightarrow$ Cat, satisfying $\mathrm{B}_{u}(B)\left(u^{\prime}\right)=B\left(u \bullet u^{\prime}\right)$.

4.3. More axioms. We now turn to generalising further constructions of HP to the general setting of playgrounds. We mentioned in Section 3 that strategies on a position $X$ should be defined as presheaves on the category of views on $X$. We will further want to generalise the decomposition theorems for strategies of $\mathrm{HP}$, which crucially rely on a property of views stated (in Section 4.4 below) as Proposition 4.27.

In order for this to work, we need to state more axioms for $\mathbb{D}$. In particular, the axioms equip $\mathbb{D}$ with a notion of player for a position $X$. Each position has a set of players, each player having a certain 'type'. Furthermore, in Section 4.4, $\mathbb{D}$ is equipped with a notion of 
view; and views have a type, too. Proposition 4.27 e.g., states that views on a position $X$ form a coproduct, over all players $x$ in $X$, of views over the type of $x$.

We first state a series of simple axioms, and then, building on these, two more complicated axioms.

Axiom. $\mathbb{D}$ is equipped with

- a full subcategory $\mathbb{I} \hookrightarrow \mathbb{D}_{h}$ of objects called individuals,

- a replete class $\mathbb{M}$ of vertical morphisms called moves, with replete subclasses $\mathbb{B}$ and $\mathbb{F}$, respectively called basic and full moves,

- a map $|-|: \operatorname{ob}(D) \rightarrow \mathbb{N}$ called the length,

satisfying the following conditions:

(P2) II is discrete. Basic moves have no non-trivial automorphisms in $D$. Vertical identities on individuals have no non-trivial endomorphisms.

(P3) (Individuality) Basic moves have individuals as both domain and codomain.

(P4) (Atomicity) For any cell $\alpha: v \rightarrow u$, if $|u|=0$ then also $|v|=0$. Up to a special isomorphism in $D$, all plays $u$ of length $n>0$ admit decompositions into $n$ moves. For any $u: X \rightarrow Y$ of length 0 , there is an isomorphism $i d_{X}^{\bullet} \rightarrow u$ as on the right in $D$.

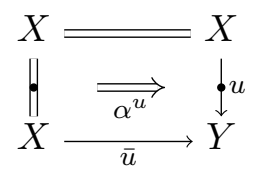

(P5) (Fibration, continued) Restrictions of moves (resp. full moves) to individuals either are moves (resp. full moves), or have length 0 .

Replete means stable under isomorphism (here in $D$ ). In (P5), restriction is w.r.t. the fibration cod: $D \rightarrow \mathbb{D}_{h}$, as explained below Proposition 4.1 .

Definition 4.7. A player in a position (i.e., object) $X$, is a pair $(d, x)$, where $d \in \mathbb{I}$ and $x: d \rightarrow X$. Let $\operatorname{Pl}(X)=\sum_{d \in \mathbb{I}} \mathbb{D}_{h}(d, X)$ be the set of players of $X$.

Example 4.8. In $\mathbb{D}^{C C S}$, individuals are representable positions $[n]$, which consist for some $n$ of a single $n$-ary player, connected to $n$ distinct channels. Importantly, for each isomorphism class of such positions we pick one representative: this makes $\mathbb{I}$ discrete by Yoneda. Furthermore, basic moves are basic seeds.

Here is a further, crucial axiom.

Definition 4.9. Let $\mathbb{B}_{0}$ be the full subcategory of $D$ having as objects basic moves and morphisms of length 0 between individuals.

Axiom. (P6) (Views) For any move $M: Y \rightarrow X$ in $\mathbb{D}_{v}$, the domain functor dom: $\mathbb{B}_{0} / M \rightarrow$ $\mathbb{I} / Y$ is an equivalence of categories.

In elementary terms, for any $y: d \rightarrow Y$ in $\mathbb{D}_{h}$ with $d \in \mathbb{I}$, there exists a cell

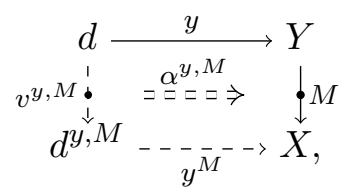

with $v^{y, M} \in \mathbb{B}_{0}$, which is unique up to canonical isomorphism of such. An isomorphism between two such tuples, say $\left(d^{\prime}, v^{\prime}, y^{\prime}, \alpha^{\prime}\right)$ and $\left(d^{\prime \prime}, v^{\prime \prime}, y^{\prime \prime}, \alpha^{\prime \prime}\right)$ is a diagram 


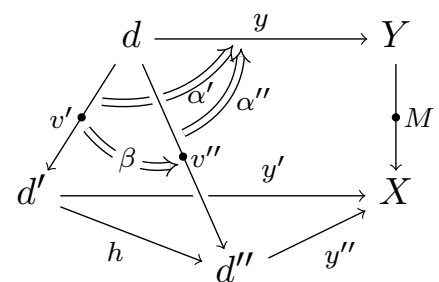

such that $\alpha^{\prime \prime} \circ \beta=\alpha^{\prime}$ (where necessarily $d^{\prime}=d^{\prime \prime}, h=i d$, and $y^{\prime}=y^{\prime \prime}$ ).

Example 4.10. This axiom is obviously satisfied by $\mathbb{D}^{C C S}$.

We then have two decomposition axioms.

Axiom. (P7) (Left decomposition) Any double cell

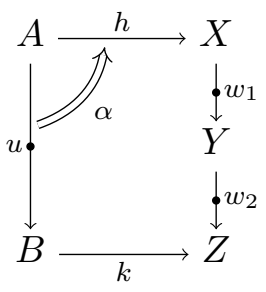

decomposes as

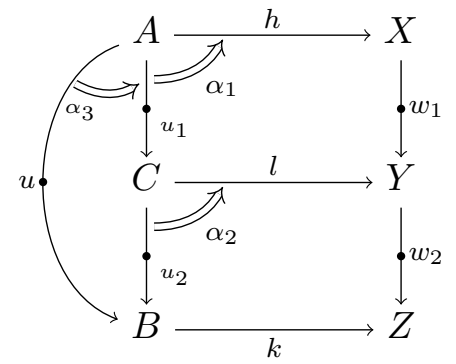

with $\alpha_{3}$ an isomorphism, in an essentially unique way.

Here is our second decomposition axiom.

Axiom. (P8) (Right decomposition) Any double cell as in the center below, where $b$ is a basic move and $M$ is a move, decomposes in exactly one of the forms on the left and right:
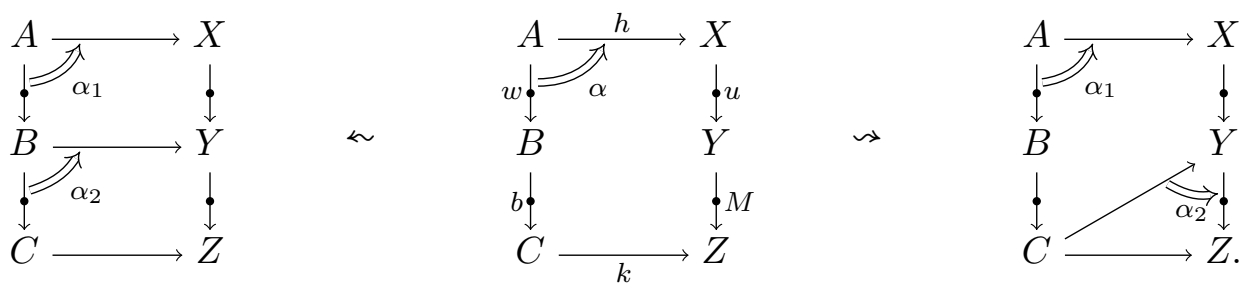

Remark 4.11. This axiom takes pseudoness rather sloppily. Indeed, the domain of the right-hand composite is not really $b \bullet w$, but rather $i d_{C}^{\bullet} \bullet(b \bullet w)$. So we actually mean $\alpha=\left(\alpha_{2} \bullet \alpha_{1}\right) \circ \lambda_{b \bullet w}^{-1}$, where $\lambda$ cancels identities on the left.

Example 4.12.

That this axiom is satisfied by $\mathbb{D}^{C C S}$ is not obvious and is proved in Section 7. However, let us disprove the more general version where $b$ is not required to be basic. Let $X$ consist of two players $x$ and $y$ sharing a channel $a$. Let $i_{y}: X \rightarrow X$ be the play where $y$ inputs on $a, o_{x}: X \rightarrow X$ be the play where $x$ outputs on $a$, and let $S: X \rightarrow X$ be the play where both players synchronise on $a$. We obtain a double cell as on the right,

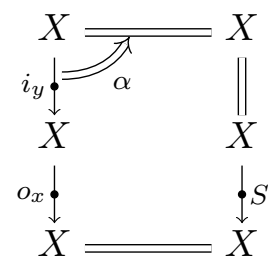
which does not decompose as in (P8). The problem here is that, on the left-hand side, the upper input by $y$ has to be mapped to $S$, which prevents any suitable decomposition. 
We now define and study views.

\subsection{Views.}

Definition 4.13. A view in $\mathbb{D}$ is a play which is specially isomorphic in $D$ to a possibly empty (vertical) composite of basic moves. I.e., if $d_{n} \stackrel{b_{n}}{\rightarrow} d_{n-1} \ldots d_{1} \stackrel{b_{1}}{\rightarrow} d_{0}$ are all basic moves, then the composite is a view. Let $\mathbb{V}$ be the full subcategory of $D$ consisting of views.

The definition includes the 'identity' view $i d_{d}^{\bullet}$. In $\mathbb{D}^{C C S}$, this of course coincides with views as defined in $\mathrm{HP}$.

Here is an important consequence of our axioms. It is a bit complicated to state, but very useful in the (more intelligible) developments on views below.

Lemma 4.14. For all plays $w: Y \rightarrow d_{n}$ and $u: X_{p} \rightarrow X_{0}$, views $v: d_{n} \rightarrow d_{0}$, and double cells $\alpha: v \bullet w \rightarrow u$, for all special isomorphisms $\gamma:\left(b_{1} \bullet\left(\ldots\left(b_{n-1} \bullet b_{n}\right) \ldots\right)\right) \rightarrow v$ and $\gamma^{\prime}: u \rightarrow$ $\left(M_{1} \bullet\left(\ldots\left(M_{p-1} \bullet M_{p}\right) \ldots\right)\right)$ decomposing $v$ and $u$ into moves, there exists a unique, strictly monotone map $f: n \cup\{0\} \rightarrow p \cup\{0\}$ with $f(0)=0$ and double cells $\beta: w \rightarrow\left(M_{f(n)+1} \bullet\left(\ldots \bullet M_{p}\right)\right)$ and $\alpha_{k}: \bar{b}_{k} \rightarrow M_{k}$ for $1 \leq k \leq f(n)$, where

$$
\bar{b}_{k}= \begin{cases}b_{i} & (\text { if } k \in \operatorname{Im}(f), \text { with } f(i)=k) \\ i d_{\operatorname{cod}\left(b_{\min \{i \in n \mid f(i)>k\}}\right)} & \text { (otherwise), }\end{cases}
$$

such that $\alpha_{1} \bullet\left(\ldots \bullet\left(\alpha_{f(n)} \bullet \beta\right)\right)=\gamma^{\prime} \circ \alpha \circ(\gamma \bullet w)$, as in
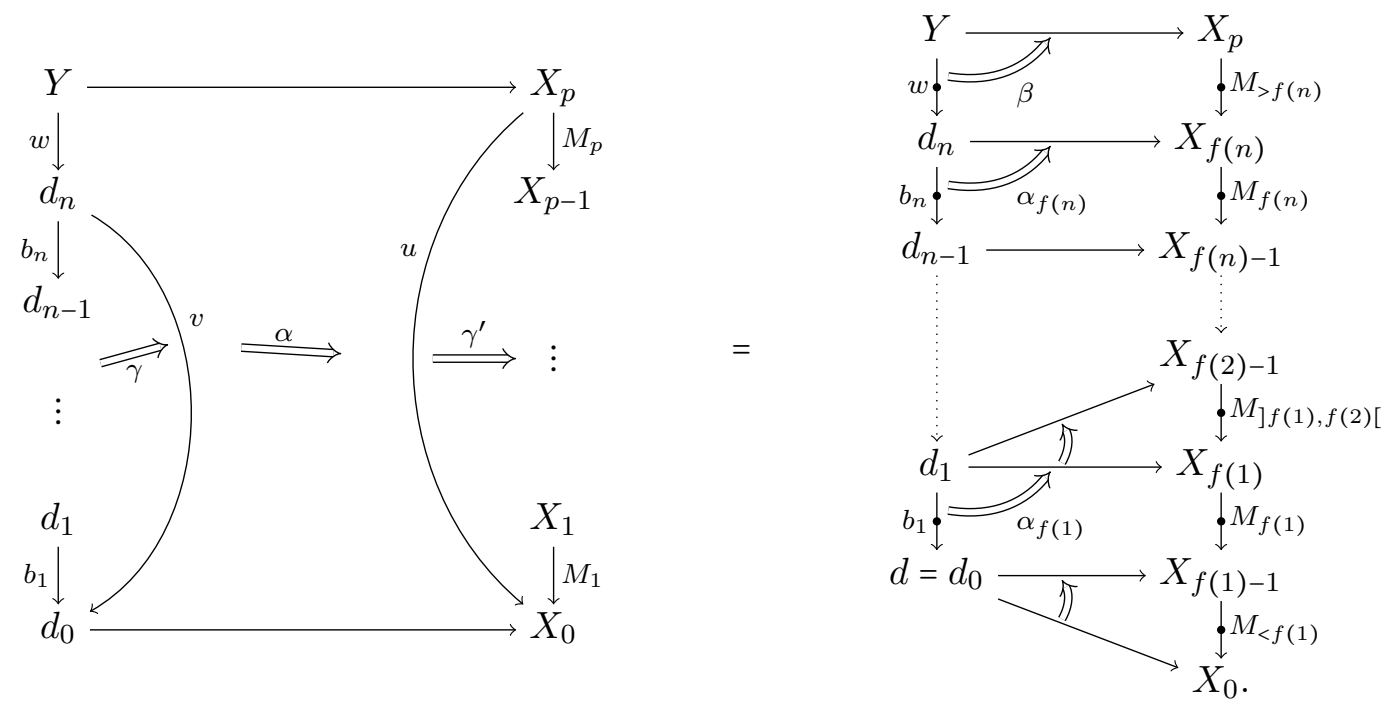

In the case $p=0$, also $n=|w|=0$, and the decomposition of $u$ should be understood as $M_{1} \bullet \ldots \bullet M_{f(n)}$ being an identity, with $M_{f(n)+1} \bullet \ldots \bullet M_{p}$ being $u$.

Remark 4.15. Only $f$ is claimed to be unique here. Furthermore, as in (P8), we are a bit sloppy regarding pseudoness. Also, in the following, we consider only the underlying map $f: n \rightarrow p$, implicitly extended with $f(0)=0$. Finally, for all $\alpha: v \bullet w \rightarrow u$, there exist $\gamma$ and $\gamma^{\prime}$ as in the lemma. This is obvious when $n$ and $p \neq 0$; we just explained it for the case $p=0$; and when $n=0$ it follows from Lemma 4.17 below. 
Proof. We proceed by lexicographic induction on the pair $(n, p)$.

If $n=0$ then our map $f: n \rightarrow p$ is the unique map $0 \rightarrow p, f(n)=0$, and we take $\beta=\gamma^{\prime} \circ \alpha \circ \gamma$. Otherwise, we apply $\left.[\mathrm{P} 8)\right]$ with $b=b_{1}, w=\left(b_{2} \bullet \ldots \bullet b_{n-1} \bullet w\right), M=M_{1}$ and $u=\left(M_{2} \bullet \ldots \bullet M_{p-1}\right)$.

- If we are in the left-hand case, $\alpha$ decomposes as $\alpha_{1} \bullet \alpha_{2}$, with $\alpha_{1}: b_{1} \rightarrow M_{1}$ and $\alpha_{2}:\left(b_{2} \bullet\right.$ $\left.\ldots \bullet b_{n} \bullet w\right) \rightarrow\left(M_{2} \bullet \ldots \bullet M_{p}\right)$. By induction hypothesis, we obtain a map $f^{\prime}: n-1 \rightarrow p-1$ and a corresponding decomposition of $\alpha_{2}$. We then let $f: n \rightarrow p$ map 1 to 1 , and $k+1$ to $f^{\prime}(k)+1$ for any $k \in(n-1)$.

- If we are in the right-hand case, we obtain a map $f^{\prime}: n \rightarrow p-1$, and return the map $k \mapsto f^{\prime}(k)+1$.

This shows existence of the desired decomposition. For uniqueness, consider any map $g: n \rightarrow$ $p$ and corresponding decomposition. Axiom (P7) entails that at each stage, $f^{-1}\{1, \ldots, k\}$ and $g^{-1}\{1, \ldots, k\}$ have the same cardinality. Indeed, otherwise, we would find isomorphic decompositions of $b_{1} \bullet \ldots \bullet\left(b_{n} \bullet w\right)$ with incompatible lengths. Thus, $f=g$.

We continue with a few easy results. Recall the family of isomorphisms $\alpha^{u}$ from Axiom (P4), indexed by vertical morphisms of length 0 . Furthermore, let us denote by $\rho_{u}: u \bullet i d_{X}^{\bullet} \rightarrow u$ and $\lambda_{u}: i d_{Y}^{\bullet} \bullet u \rightarrow u$ the coherence isomorphisms from $\mathbb{D}_{v}$ for cancelling vertical identities.

Lemma 4.16. For any $u: X \rightarrow Y$ of length 0 , there is an isomorphism

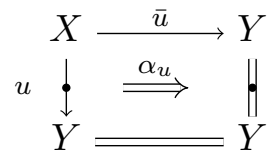

in $Ð$, such that $\alpha_{u} \bullet \alpha^{u}=\lambda_{u}^{-1} \circ \rho_{u}$ and $\alpha_{u} \circ \alpha^{u}=i d_{\bar{u}}^{\bullet}$.

Proof. Pose $\alpha_{u}=i d_{\bar{u}}^{\bullet} \circ\left(\alpha^{u}\right)^{-1}$.

Lemma 4.17. If $b: d \rightarrow d^{\prime}$ has length 0 , then $d=d^{\prime}, \bar{b}=i d_{d}$, and $\alpha_{b}$ and $\alpha^{b}$ are horizontal inverses.

Proof. By (P2).

Lemma 4.18. $\mathbb{B}_{0}$ (Definition 4.9 ) is a groupoid.

Proof. This means that any $\alpha: b \rightarrow b^{\prime}$ in $\mathbb{B}_{0}$ is an isomorphism. Let $b: d_{1} \rightarrow d_{2}$ and $b^{\prime}: d_{1}^{\prime} \rightarrow d_{2}^{\prime}$. Existence of $\alpha$ entails $d_{1}=d_{1}^{\prime}$ and $d_{2}=d_{2}^{\prime}$, by (P2).

If $b^{\prime} \in \mathbb{B}$, then $\alpha$ and $i d_{b^{\prime}}$ are both mapped by $\operatorname{dom}: \mathbb{B}_{0} / b^{\prime} \rightarrow \mathbb{I} / d_{1}^{\prime}$ to $\operatorname{dom}(\alpha)=i d_{d_{1}^{\prime}}$. By (P6), there is thus a unique isomorphism $\gamma: b \rightarrow b^{\prime}$ in $D$ such that $i d_{b^{\prime}} \circ \gamma=\alpha$, i.e., $\gamma=\alpha$. This shows that $\alpha$ is an iso.

If $b^{\prime}$ has length 0 , then by (P4) we furthermore have $|b|=0$ and $d_{1}=d_{2}=d_{1}^{\prime}=d_{2}^{\prime}$. Moreover, the composite $\alpha_{b^{\prime}} \circ \alpha \circ \alpha^{b}$ (with $\alpha_{b^{\prime}}$ and $\alpha^{b}$ as in Lemma 4.16 and (P4)) is an endomorphism of $i d_{d_{1}}^{\bullet}$, hence $i d_{i d_{d_{1}}^{\bullet}}$ by (P2). It is thus an isomorphism, hence so is $\alpha^{b^{\prime}} \circ \alpha_{b^{\prime}} \circ \alpha \circ \alpha^{b} \circ \alpha_{b}$, which is equal to $\alpha$ by two applications of Lemma 4.17.

Lemma 4.19. In any category $\mathbb{C}$, for any object $c$ isomorphic to an object $d$ such that $d$ has no non-trivial endomorphisms, $c$ does not have any non-trivial endomorphisms either.

Proof. By the Yoneda lemma, we have $\mathbb{C}(c, c) \cong \mathbb{C}(c, d) \cong \mathbb{C}(d, d) \cong 1$. 
Lemma 4.20. Any groupoid $\mathbb{C}$ whose objects have no non-trivial endomorphisms is an equivalence relation.

Proof. For any objects $c$ and $d$, we have that if $\mathbb{C}(c, d)$ is non-empty then $c$ and $d$ are isomorphic, so by Yoneda $\mathbb{C}(c, d) \cong \mathbb{C}(c, c) \cong 1$.

Corollary 4.21. $\mathbb{B}_{0}$ is an equivalence relation.

This adds to Lemma 4.18 that there is at most one morphism between any two objects.

Proof. By Lemma 4.19 and (P4), its objects have no non-trivial automorphisms, which in a groupoid is the same as having no non-trivial endomorphisms. By the last result, $\mathbb{B}_{0}$ is an equivalence relation.

This leads to a better understanding of $\mathbb{V}$.

Lemma 4.22. Consider any morphism of views $\alpha: v \rightarrow v^{\prime}$, with isomorphisms $\gamma:\left(b_{1} \bullet \ldots \bullet\right.$ $\left.b_{n}\right) \rightarrow v$ and $\gamma^{\prime}: v^{\prime} \rightarrow\left(b_{1}^{\prime} \bullet \ldots \bullet b_{n^{\prime}}^{\prime}\right)$, for basic moves $b_{i}: d_{i} \rightarrow d_{i-1}$ and $b_{j}^{\prime}: d_{j}^{\prime} \rightarrow d_{j-1}^{\prime}$ for all $i \in n$ and $j \in n^{\prime}$. We have $n=n^{\prime}, d_{i-1}=d_{i-1}^{\prime}$ for all $i \in n+1$, and there exist unique isomorphisms $\alpha_{i}: b_{i} \rightarrow b_{i}^{\prime}$ such that $\gamma^{\prime} \circ \alpha \circ \gamma=\left(\alpha_{n} \bullet \ldots \bullet \alpha_{1}\right)$, as in
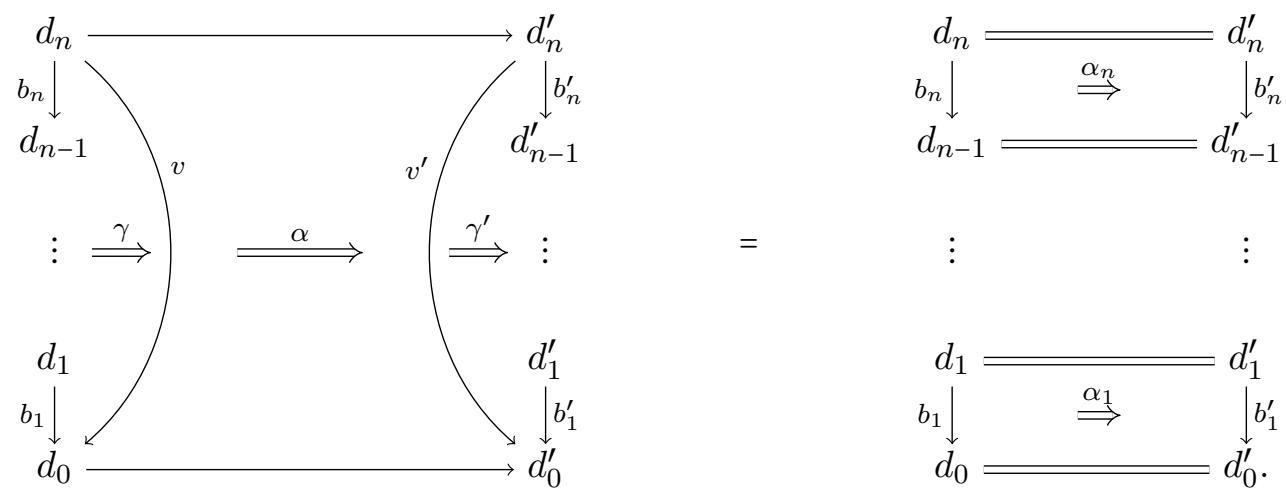

Proof. Applying Lemma 4.14 with $w=i d_{d_{n}}^{\bullet}$ yields $f: n \rightarrow n^{\prime}$ which by Corollary 4.21 and (P4) has to be a bijection. This yields the desired $\alpha_{i}$ 's, which are unique by Corollary 4.21 again.

This entails:

Corollary 4.23. $\mathbb{V}$ is an equivalence relation, compatible with length. moves.

Here is an analogue of (P6) for general plays and views instead of just moves and basic

Proposition 4.24. For any $y: d \rightarrow Y$ in $\mathbb{D}_{h}$ with $d \in \mathbb{I}$, and any $u: Y \rightarrow X$ in $\mathbb{D}_{v}$, there exists a cell

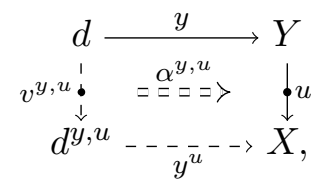

with $v^{y, u}$ a view, which is unique up to canonical isomorphism of such.

Proof. We find $v^{y, u}$ by repeated application of (P6), For essential uniqueness, by repeated application of (P6), we find an isomorphism between any two such views, which by Corollary 4.23 is unique. 
We continue with an analogue of (P8)

Proposition 4.25. Any double cell

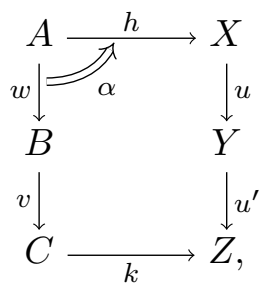

where $v$ is a view, decomposes in exactly one of the following forms:
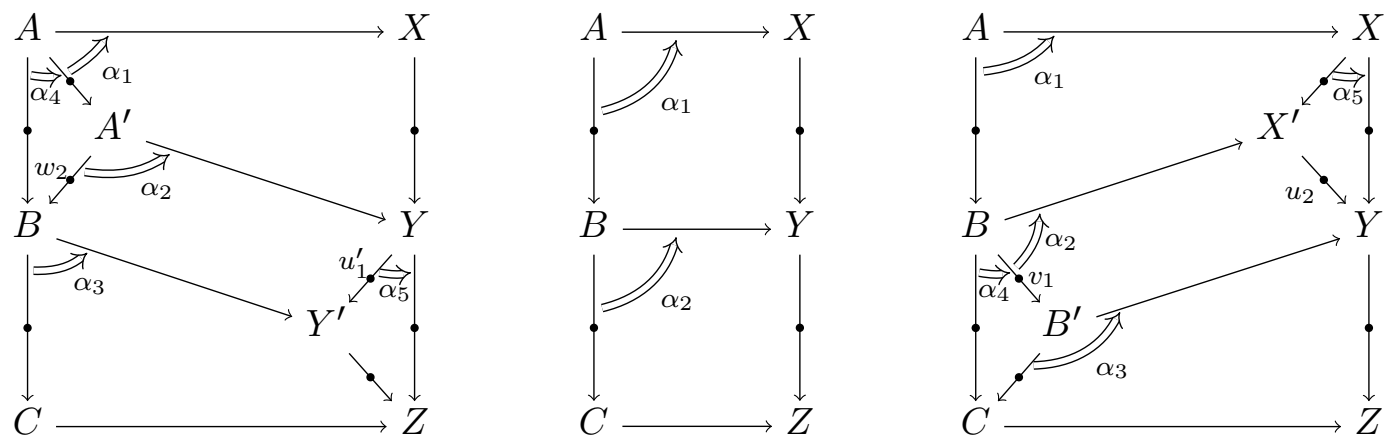

with $\left|w_{2}\right|>0,\left|v_{1}\right|>0$, and $\alpha_{4}$ and $\alpha_{5}$ iso in $Đ$.

A possible reading of this is that in the left and middle cases, the whole of $v$ embeds into $u^{\prime}$. In the left case, a non-trivial part of $w$ embeds into $u^{\prime}$. In the right case, a non-trivial part of $v$ embeds into $u$.

Proof. Choose decompositions of $u^{\prime}$ and $u$ as $M_{1} \bullet \ldots \bullet M_{p}$ and $M_{p+1} \bullet \ldots \bullet M_{p+q}$, respectively, and of $v$ as $b_{1} \bullet \ldots \bullet b_{n}$. Apply Lemma 4.14 to obtain $f: n \rightarrow p+q$. If $f(n)>p$, we are in the right-hand case. If $f(n)=p$, we are in the middle case. If $f(n)=r<p$, let $u_{2}^{\prime}=M_{1} \bullet \ldots M_{r}$ and $u_{1}^{\prime}=M_{r+1} \bullet \ldots \bullet M_{p}$. Lemma 4.14 provides $\beta: w \rightarrow u_{1}^{\prime} \bullet u$ and $\gamma: v \rightarrow u_{2}^{\prime}$ such that $\gamma \bullet \beta=\alpha$. Applying (P7) to $\beta$ gives a decomposition of $\alpha$ as on the left below
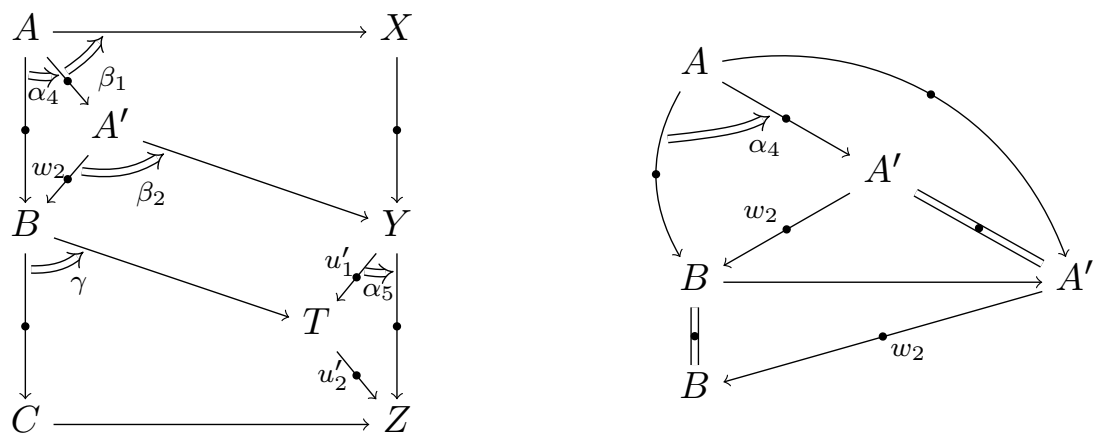

with $\alpha_{4}$ and $\alpha_{5}$ isos. If $\left|w_{2}\right| \neq 0$, then we are in the left-hand case of the proposition, and the middle case is impossible by essential uniqueness in (P7). Otherwise, we may decompose $\alpha_{4}$ as on the right by atomicity (empty cells are given by coherence or (P4)], so we are in the middle case of the proposition. 
Lastly, we need a few more definitions before Proposition 4.27.

Definition 4.26. Let $\mathbb{E}^{\mathbb{V}}$ be the full subcategory of $\mathbb{E}$ consisting of views.

Consider, for any $X$, the comma category $\mathbb{E}_{X}$ induced by the vertical codomain functor cod: $\mathbb{E} \rightarrow \mathbb{D}_{h}$ mapping (4.1) to $k$ (following notation from Section 2.1). Similarly, consider $\mathbb{E}_{X}^{\mathbb{V}}$. Concretely, an object of $\mathbb{E}_{X}^{\mathbb{V}}$ is a pair of a view $v: d^{\prime} \rightarrow d$, and a player $x: d \rightarrow X$ of $X$. A morphism $\left(v_{1}, x_{1}\right) \rightarrow\left(v_{2}, x_{2}\right)$ is a morphism $(w, \alpha): v_{1} \rightarrow v_{2}$ in $\mathbb{E}^{\mathbb{V}}$, such that $x_{2} \circ \operatorname{cod}(\alpha)=x_{1}$.

Recall now from above Definition 4.6 the pullback category $\mathbb{E}(X)$. It is isomorphic to the full subcategory of $\mathbb{E}_{X}$ consisting of pairs $(u, x)$ where $x=i d_{X}$. Similarly, we have $\mathbb{E}^{\mathbb{V}}(X)$, which is empty unless $X$ is an individual.

Proposition 4.27. We have

(i) The inclusion $\mathbb{E}^{\mathbb{V}}(d) \hookrightarrow \mathbb{E}_{d}^{\mathbb{V}}$ mapping $v$ to $\left(v, i d_{d}\right)$ is an isomorphism of categories.

(ii) The inclusion $\sum_{(d, x) \in \operatorname{Pl}(X)} \mathbb{E}^{\mathbb{V}}(d) \hookrightarrow \mathbb{E}_{X}^{\mathbb{V}}$ mapping $((d, x), v)$ to $(v, x)$ is an isomorphism of categories.

(iii) $\mathbb{E}^{\mathbb{V}}(d)$ is a preorder.

Proof. First, because $\mathbb{I}$ is discrete, $\mathbb{D}_{h}(d, d)=\left\{i d_{d}\right\}$, hence (i). For (ii), the functor $\mathbb{E}_{X}^{\mathbb{V}} \rightarrow$ $\sum_{(d, x) \in \operatorname{Pl}(X)} \mathbb{E}^{\mathbb{V}}(d)$ mapping any $(v, x)$ to $((d, x), v)$, with $v: d^{\prime} \rightarrow d$ a view and $x: d \rightarrow X$ a player, is inverse to the given functor. Finally, consider any two morphisms $v_{1} \rightarrow v_{2}$ in $\mathbb{E}^{\mathbb{V}}(d)$, say

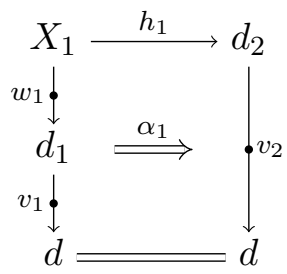

and

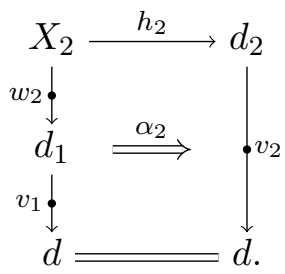

Fixing decompositions of $v_{1}$ and $v_{2}$ into basic moves, we obtain by Lemmas 4.14 and 4.22 that $\alpha_{1}$ and $\alpha_{2}$ respectively decompose as

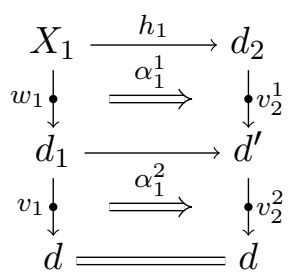

and

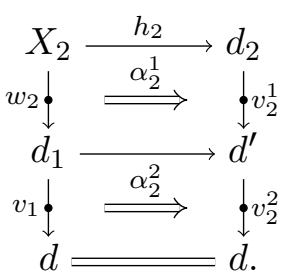

By Corollary 4.23, $\alpha_{1}^{2}=\alpha_{2}^{2}$. Furthermore, we conclude by (P1) and the quotienting (4.2) in the definition of $\mathbb{E}$ that both morphisms are equal in $\mathbb{E}^{\mathbb{V}}\left(\overline{d)}\right.$ to $\alpha_{1}^{2} \bullet i d_{v_{2}^{1}}$.

\subsection{From behaviours to strategies.}

Definition 4.28. The category $S S_{X}$ of strategies on $X$ is the category $\widehat{\mathbb{E}_{X}^{\mathbb{V}}}$ of presheaves of finite ordinals on $\mathbb{E}_{X}^{\mathbb{V}}$. 
Example 4.29. On $\mathbb{D}^{C C S}, \mathbb{E}_{X}^{\mathbb{V}}$ as defined here yields a category equivalent to the definition in HP, so the categories of strategies are also equivalent (even isomorphic because ford contains no non-trivial automorphism).

The rest of this section develops some structure on strategies, which is needed for constructing the LTS in Section [5.2. We start by extending the assignment $X \mapsto S S_{X}$ to a pseudo double functor $\mathbb{D}^{o p} \rightarrow \mathbb{Q}$ Cat, where $\mathbb{Q}$ Cat is Ehresmann's double category of quintets on the 2-category Cat:

Definition 4.30. $\mathbb{Q}$ Cat has small categories as objects, functors as both horizontal and vertical morphisms, and natural transformations as double cells.

Actually, our first step is to extend the assignment $X \mapsto \mathbb{E}_{X}^{\mathbb{V}}$ to pseudo double functor $\mathbb{D} \rightarrow \mathbb{Q}$ Cat. Define the action of a horizontal map $h: X \rightarrow X^{\prime}$ to map any object $(v, x)$ of $\mathbb{E}_{X}^{\mathbb{V}}$ to $(v, h \circ x)$, and any morphism to itself viewed as a morphism in $\mathbb{E}_{X^{\prime}}^{\mathbb{V}}$. (This functor is induced by universal property of $\mathbb{E}_{X}^{\mathbb{V}}$ as a comma category.) This defines a functor $\mathbb{E}_{-}^{\mathbb{V}}: \mathbb{D}_{h} \rightarrow$ Cat. The pseudo functor $\mathbb{D}_{v} \rightarrow$ Cat is a bit harder to construct. For any $u: Y \rightarrow X$ in $\mathbb{D}_{v}$ and $y: d \rightarrow Y$, the cell $\alpha^{y, u}$ from Proposition 4.24 induces a functor $\Sigma_{v^{y, u}}: \mathbb{E}^{\mathbb{V}}(d) \rightarrow \mathbb{E}^{\mathbb{V}}\left(d^{y, u}\right)$ mapping any $v: d^{\prime} \rightarrow d$ to $v^{y, u} \bullet v$. Composing with the coproduct injection $i n j_{d^{y, u}, y^{u}}: \mathbb{E}^{\mathbb{V}}\left(d^{y, u}\right) \hookrightarrow \sum_{\left(d^{\prime \prime}, x\right) \in \operatorname{Pl}(X)} \mathbb{E}^{\mathbb{V}}\left(d^{\prime \prime}\right)$, because $\mathbb{E}_{X}^{\mathbb{V}} \cong \sum_{\left(d^{\prime \prime}, x\right) \in \operatorname{Pl}(X)} \mathbb{E}^{\mathbb{V}}\left(d^{\prime \prime}\right)$, we obtain functors

$$
\mathbb{E}^{\mathbb{V}}(d) \stackrel{\Sigma_{v y, u}}{\longrightarrow} \mathbb{E}^{\mathbb{V}}\left(d^{y, u}\right) \stackrel{i n j_{d} y, u, y}{\longrightarrow} \mathbb{E}_{X}^{\mathbb{V}}
$$

whose copairing defines a functor $\mathbb{E}_{u}^{\mathbb{V}}: \mathbb{E}_{Y}^{\mathbb{V}} \rightarrow \mathbb{E}_{X}^{\mathbb{V}}$.

Now, for any cell as on the left below, we obtain by Proposition 4.24 a canonical natural isomorphism as on the right
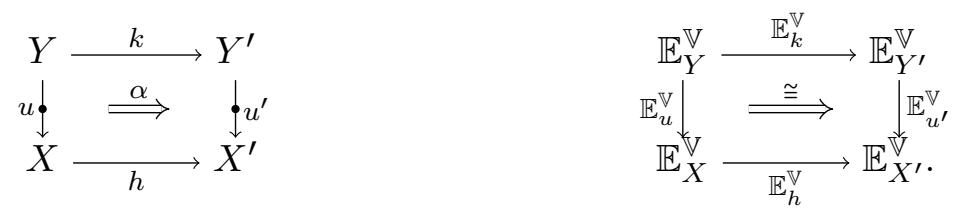

By canonicity of the above double cell, we have

Proposition 4.31. This assignment defines a pseudo double functor $\mathbb{E}_{-}^{\mathbb{V}}: \mathbb{D} \rightarrow \mathbb{Q}$ Cat.

Definition 4.32. Let the opposite $\mathbb{D}^{o p}$ of a pseudo double category $\mathbb{D}$ be obtained by reversing both vertical and horizontal arrows, and hence double cells.

We obtain:

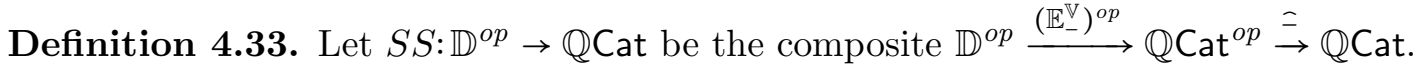

As a shorthand, we denote $S S(f)(S)$ by $S \cdot f$ for $f$ horizontal or vertical. Concretely, for any horizontal $h: Z \rightarrow X, S \cdot h$ satisfies

$$
(S \cdot h)(v, z)=S(v, h \circ z),
$$

whereas for any vertical $u: Y \rightarrow X, S \cdot u$ satisfies

$$
(S \cdot u)(v, y)=S\left(v^{y, u} \bullet v, y^{u}\right) .
$$

We conclude this section by constructing the extension functor from strategies to behaviours, in arbitrary playgrounds. 
Recall that strategies on a position $X$ are presheaves of finite ordinals on $\mathbb{E}_{X}^{\mathbb{V}}$, and that behaviours are presheaves of finite sets on $\mathbb{E}(X)$. To go from the former to the latter, we use $\mathbb{E}_{X}$ as a bridge. Recall from Section 2.1 that objects of $\mathbb{E}_{X}^{\mathbb{V}}$ are diagrams of the shape $d^{\prime} \stackrel{v}{\rightarrow} d \stackrel{x}{\rightarrow} X$, with $v$ a view, and that objects of $\mathbb{E}(X)$ are just plays $Y \stackrel{u}{\rightarrow} X$. The idea here is that on the one hand $\mathbb{E}_{X}^{\mathbb{V}}$ is richer than $\mathbb{E}(X)$, in that its objects may be plays on subpositions of $X$, whereas objects of $\mathbb{E}(X)$ are plays on the whole of $X$. But on the other hand, $\mathbb{E}(X)$ is richer than $\mathbb{E}_{X}^{\mathbb{V}}$ because its objects may be arbitrary plays, whereas objects of $\mathbb{E}_{X}^{\mathbb{V}}$ have to be views. $\mathbb{E}_{X}$ contains both $\mathbb{E}_{X}^{\mathbb{V}}$ and $\mathbb{E}(X)$, its objects being diagrams $Y \stackrel{u}{\rightarrow} Z \stackrel{h}{\rightarrow} X$, for arbitrary plays $u$.

First, let $k_{X}: \widetilde{\mathbb{E}_{X}^{\mathbb{V}}} \rightarrow \overline{\mathbb{E}_{X}^{\mathbb{V}}}$ denote postcomposition with ford $\hookrightarrow$ set. Because views form a full subcategory of $D$, all embeddings $i_{X}: \mathbb{E}_{X}^{\mathbb{V}} \hookrightarrow \mathbb{E}_{X}$ are also full. This entails:

Lemma 4.34. For all $X$, right Kan extension $\left(i_{X}^{o p}\right)_{\star}: \overline{\mathbb{E}_{X}^{\mathbb{V}}} \hookrightarrow \overline{\mathbb{E}_{X}}$ along $i_{X}^{o p}$ is well-defined, full, and faithful.

Proof. One easily shows that, when defined, right extension along a full and faithful functor is full and faithful.

It remains to show that the considered right extensions exist. It is well-known [38] that the right Kan extension of any $S \in \overline{\mathbb{E}_{X}^{\mathbb{V}}}$ maps any $(u, h)$ to the limit of the functor $\left(\mathbb{E}_{X}^{\mathbb{V}} /(u, h)\right)^{o p} \rightarrow\left(\mathbb{E}_{X}^{\mathbb{V}}\right)^{o p} \stackrel{S}{\rightarrow}$ set, if the latter exists. Since finite limits exist in set (though not in ford, which explains why we use set instead of ford for extending strategies), it is enough to prove that each $\mathbb{E}_{X}^{\mathbb{V}} /(u, h)$ is essentially finite, i.e., equivalent to a finite category. This is proved in the next lemma.

Lemma 4.35. For any play $u: Z \rightarrow Y$ and horizontal $h: Y \rightarrow X$, the category $\mathbb{E}_{X}^{\mathbb{V}} /(u, h)$ is essentially finite.

For this lemma to hold, we need more axioms.

Axiom. (P9) (Finiteness) For any position $X$, there are only finitely many players, i.e., the category $\mathbb{I} / X$ is finite.

Proof of Lemma 4.35. Let us fix a pair $(u, h)$. By Proposition 4.27, $\mathbb{E}_{X}^{\mathbb{V}} /(u, h)$ is a preorder, so we just need to prove that its object set is essentially finite. Now, letting $n=|u|$, we fix a decomposition of $u$ into moves, say $Z=Y_{n} \stackrel{M_{n}}{\rightarrow} Y_{n-1} \ldots Y_{1} \stackrel{M_{1}}{\rightarrow} Y_{0}$. For any morphism $\alpha:(v, x) \rightarrow(u, h)$ in $\mathbb{E}_{X}$, by Lemma 4.14, $m=|v|$ may not exceed $n$. Furthermore, by Lemma 4.14, Proposition 4.24, and our quotienting (4.2), any such $\alpha$ is determined up to isomorphism by $m$, a strictly monotone map $f: m \rightarrow n$, and a player $y$ of $Y_{f(m)}$. Because such triples $(m, f, y)$ are in finite number, $\mathbb{E}_{X}^{\mathbb{V}} /(u, h)$ is essentially finite.

This concludes the proof of Lemma 4.34; right Kan extension along $i_{X}^{o p}:\left(\mathbb{E}_{X}^{\mathbb{V}}\right)^{o p} \hookrightarrow \mathbb{E}_{X}^{o p}$ yields a full and faithful functor. We now design the second half of our bridge from $\mathbb{E}_{X}^{\mathbb{V}}$ to $\mathbb{E}(X)$ via $\mathbb{E}_{X}$. Consider the embedding $j_{X}: \mathbb{E}(X) \rightarrow \mathbb{E}_{X}$ mapping any $u$ to $\left(u, i d_{X}\right)$. Restriction along $\left(j_{X}\right)^{o p}$ defines a functor $\Delta_{\left(j_{X}\right)^{o p}}: \overline{\mathbb{E}_{X}} \rightarrow \overline{\mathbb{E}(X)}$.

Recall from Definition 4.6 the notion of behaviour.

Definition 4.36. For any $X$, let the extension functor ext ${ }_{X}: S S_{X} \rightarrow \mathrm{B}_{X}$ be the composite

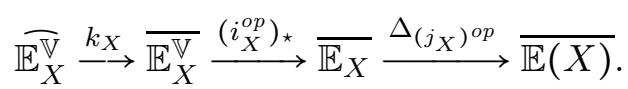


We call a behaviour on $X$ innocent when it is in the essential image of ext ${ }_{X}$.

Notation: when $X$ is clear from context, we abbreviate $\operatorname{ext}_{X}(S)$ as $\bar{S}$.

Remark 4.37. The calculations of Section 3.4 .2 carry over unchanged to the new setting.

Finally, the definitions of Section 3.5 apply more or less verbatim to the playground $\mathbb{D}^{C C S}$, yielding a semantic fair testing equivalence which coincides with that of $\mathrm{HP}$.

\section{Playgrounds: transition systems}

In the previous section, we have defined behaviours and strategies, and constructed the extension functor from the former to the latter. In this section, we first build on this to state decomposition theorems, which lead to a syntax and an LTS for strategies. Then, we define our second LTS, and relate the two by a strong, functional bisimulation.

5.1. A syntax for strategies. Let us begin by proving in the abstract setting of playgrounds analogues of the decomposition results of $\mathrm{HP}$, in particular that strategies form a terminal coalgebra for a certain polynomial functor. This is equivalent to saying that they are essentially infinite terms in a typed grammar. We use this in the next section to define our LTS $\mathcal{S}_{\mathbb{D}}$, and study transitions therein.

First, we have spatial decomposition:

Proposition 5.1. The functor $S S_{X} \rightarrow \prod_{(d, x) \in \operatorname{Pl}(X)} S S_{d}$ given at $(d, x)$ by $S S(x): S S_{X} \rightarrow$ $S S_{d}$ is an isomorphism of categories.

Proof. We have:

$$
\begin{aligned}
S S_{X} & =\operatorname{Cat}\left(\left(\mathbb{E}_{X}^{\mathbb{V}}\right)^{o p}, \text { ford }\right) \\
& \cong \operatorname{Cat}\left(\sum_{(d, x) \in \operatorname{Pl}(X)} \mathbb{E}^{\mathbb{V}}(d)^{o p}, \text { ford }\right) \quad \text { (by Proposition 4.27) } \\
& \cong \prod_{(d, x) \in \operatorname{Pl}(X)} \operatorname{Cat}\left(\mathbb{E}^{\mathbb{V}}(d)^{o p}, \text { ford }\right) \\
& =\prod_{(d, x) \in \operatorname{Pl}(X)} S S_{d} .
\end{aligned}
$$

For any $S \in S S_{X}$, let $S \cdot x$ denote the strategy on $d$ corresponding to $(d, x)$ accross the isomorphism.

The second decomposition result is less straightforward, but goes through essentially as in the concrete case. Let us be a bit more formal here than in Section 3.4.3. by showing that strategies form a terminal coalgebra for some endofunctor on Set ${ }^{\mathbb{I}}$. We start by defining the relevant endofunctor.

Definition 5.2. Let $[\mathbb{B}]_{d}$ denotes the set of all isomorphism classes of basic moves from $d$ (i.e., with vertical codomain $d$ ).

Definition 5.3. Let $G:$ Set $^{\mathbb{I}} \rightarrow$ Set $^{\mathbb{I}}$ be the functor mapping any family $U$ to

$$
(G(U))_{d}=\left(\prod_{b \in[\mathbb{B}]_{d}} U_{\mathrm{dom}(b)}\right)^{\star},
$$

where $(-)^{\star}$ denotes finite sequences. 
Remark 5.4. This functor is polynomial in the sense of Kock [31], as

$$
(G(U))_{d}=\sum_{n \in \mathbb{N}}\left(\prod_{i \in n, b \in[\mathbb{B}]_{d}} U_{\operatorname{dom}(b)}\right) .
$$

We now show that strategies, viewed as the $\mathbb{I}$-indexed family $\left(\operatorname{ob}\left(S S_{d}\right)\right)_{d \in \mathbb{I}}$, form a terminal $G$-coalgebra. We drop the ob(-) for readability.

Definition 5.5. For any $S \in S S_{d}$ and $\sigma \in S\left(i d_{d}^{\bullet}\right)$, let the restriction $S_{\mid \sigma} \in S S_{d}$ of $S$ to $\sigma$ be defined by the fact that $S_{\mid \sigma}(v)=\left\{\sigma^{\prime} \in S(v) \mid S\left(!_{v}\right)\left(\sigma^{\prime}\right)=\sigma\right\}$.

(Here, we freely use the isomorphism $\mathbb{E}_{d}^{\mathbb{V}} \cong \mathbb{E}^{\mathbb{V}}(d)$ from Proposition 4.27, and let $!_{v}$ denote the unique morphism $i d_{d}^{\bullet} \rightarrow v$ in $\mathbb{E}^{\mathbb{V}}(d)$.)

In view of Remark [5.4 $(G(S S))_{d}=\sum_{n \in \mathbb{N}}\left(\prod_{b \in[\mathbb{B}]_{d}} S S_{\operatorname{dom}(b)}\right)^{n}$. We thus may define the $G$-coalgebra structure $\partial: S S \rightarrow G(S S)$ in $\operatorname{Set}^{\mathbb{I}}$ of strategies as follows.

Definition 5.6. Let, for all $d \in \mathbb{I}, \partial_{d}: S S_{d} \rightarrow \sum_{n}\left(\prod_{b \in[\mathbb{B}]_{d}} S S_{\mathrm{dom}(b)}\right)^{n}$ send any $S \in S S_{d}$ to $n=S\left(i d_{d}^{\bullet}\right)$ and the map

$$
\begin{aligned}
S\left(i d_{d}^{\bullet}\right) & \rightarrow \prod_{b \in[\mathbb{B}]_{d}} S S_{\mathrm{dom}(b)} \\
\sigma & \mapsto b \mapsto\left(S_{\mid \sigma}\right) \cdot b .
\end{aligned}
$$

Here, we view the ordinal $S\left(i d_{d}^{\bullet}\right)$ as a natural number, and the given map $S\left(i d_{d}^{\bullet}\right) \rightarrow$ $\prod_{b \in[\mathbb{B}]_{d}} S S_{\mathrm{dom}(b)}$ as a list of elements of $\prod_{b \in[\mathbb{B}]_{d}} S S_{\mathrm{dom}(b)}$. We further use the action of $b$ on $S$, as below Definition 4.33. We have:

Theorem 5.7. The map $\partial: S S \rightarrow G(S S)$ makes $S S$ into a terminal G-coalgebra.

This intuitively means that strategies, on individuals, are infinite terms for the following typed grammar with judgements $d \vdash_{\mathrm{D}} D$ and $d \vdash S$, where $D$ is a definite strategy and $S$ is a strategy

$$
\frac{\ldots d^{\prime} \vdash S_{b} \ldots\left(\forall b: d^{\prime} \rightarrow d \in[\mathbb{B}]_{d}\right)}{d \vdash_{\mathrm{D}}\left\langle\left(S_{b}\right)_{b \in[\mathbb{B}]_{d}}\right\rangle} \quad \frac{\ldots d \vdash_{\mathrm{D}} D_{i} \ldots(\forall i \in n)}{d \vdash \bigoplus_{i \in n} D_{i}}(n \in \mathbb{N}) .
$$

Semantically, definite strategies correspond to strategies $S$ such that $S\left(i d_{d}^{\bullet}\right)=1$, which will play a crucial role in the LTS below.

The rest of this section is a proof of Theorem 5.7 .

First of all, we construct an inverse to $\partial$.

Definition 5.8. Consider $B=\left(B_{1}, \ldots, B_{n}\right) \in(G(S S))_{d}$. For any view $v: d^{\prime} \rightarrow d$, define $\partial^{\prime}(B) \in S S_{d}$ by

and on morphisms

$$
\partial^{\prime}(B)(v)= \begin{cases}n & \text { if } v=i d_{d}^{\bullet} \\ \sum_{i \in n} B_{i}(b)\left(v^{\prime}\right) & \text { if } v=b \bullet v^{\prime}\end{cases}
$$

$$
\partial^{\prime}(B)\left(v \stackrel{(w, \alpha)}{\longrightarrow} v^{\prime}\right)(\sigma)= \begin{cases}i & \text { if } v^{\prime}=i d_{d}^{\bullet} \text { and } \sigma=i \in n \\ & \text { or if } v=i d_{d}^{\bullet} \text { and } \sigma=(i, x) \\ \left(i, B_{i}(b)\left(w, \alpha_{1}\right)(x)\right) & \text { if } v=b \bullet v_{1} \text { and } \sigma=(i, x)\end{cases}
$$

where in the last clause necessarily $v^{\prime} \cong b^{\prime} \bullet v_{1}^{\prime}$ and Lemma 4.14 yields $\alpha_{b}: b \stackrel{\cong}{\rightarrow} b^{\prime}$ and $\alpha_{1}: v_{1} \bullet w \rightarrow v_{1}^{\prime}$ such that $\alpha_{b} \bullet \alpha_{1}=\alpha$. 
Lemma 5.9. We have $\partial^{\prime}=\partial^{-1}$.

Proof. Starting from a strategy $S \in S S_{d}$, let $n=S\left(i d_{d}^{\bullet}\right)$, and $B_{i}(b)=\left(S_{\mid i}\right) \cdot b$, for any $d^{\prime} \stackrel{b}{\bullet} d$. We have $\partial S=\left(B_{1}, \ldots, B_{n}\right)$, and thus $\partial^{\prime}(\partial S)(v)=n$ if $v=i d_{d}^{\bullet}$, and $\partial^{\prime}(\partial S)(v)=$ $\sum_{i \in n}\left(S_{\mid i}\right)\left(b \bullet v^{\prime}\right)=S(v)$ if $v=b \bullet v^{\prime}$, as desired.

Conversely, starting from $B=\left(B_{1}, \ldots, B_{n}\right) \in(G(S S))_{d}$, let $S=\partial^{\prime} B$. We have that $\partial S$ has length $n$, and its $i$ th component maps any $b: d^{\prime} \rightarrow d$ to the strategy mapping any $v^{\prime}: d^{\prime \prime} \rightarrow d^{\prime}$ to the strategy $\left(S_{\mid i}\right) \cdot b$. Thus, $(\partial S)_{i}(b)\left(v^{\prime}\right)=\left(\left(S_{\mid i}\right) \cdot b\right)\left(v^{\prime}\right)=\left(S_{\mid i}\right)\left(b \bullet v^{\prime}\right)$. But by definition, this is equal to $B_{i}(b)\left(v^{\prime}\right)$, as desired.

Consider any $G$-coalgebra $a: U \rightarrow G U$.

We define by induction on $N$ a sequence of maps $f_{N}: U \rightarrow S S$, such that for any $d$ and $u \in U_{d}$ the $f_{n+N}(u)$ 's agree on views of length $\leq n$. I.e., for any $d \in \mathbb{I}, u \in U_{d}$, view $v$ of length less than $n$, and any $N, f_{n+N}(u)(v)=f_{n}(u)(v)$, and similarly the action of $f_{n+N}(u)$ on morphisms between such views is the same as that of $f_{n}(u)$.

To start the induction, take $f_{0}(u)$ to be the strategy mapping $i d_{d}^{\bullet}$ to $|a(u)|$, i.e., the length of $a(u) \in\left(\prod_{b} U_{\operatorname{dom}(b)}\right)^{\star}$, and all other views to 0 .

Furthermore, given $f_{N}$, define $f_{N+1}$ to be

$$
U \stackrel{a}{\rightarrow} G U \stackrel{G\left(f_{N}\right)}{\longrightarrow} G(S S) \stackrel{\partial^{-1}}{\longrightarrow} S S .
$$

In other words, $f_{N}$ is

$U \stackrel{a}{\rightarrow} G U \stackrel{G(a)}{\longrightarrow} \ldots G^{N-1} U \stackrel{G^{N-1} a}{\longrightarrow} G^{N} U \stackrel{G^{N} f_{0}}{\longrightarrow} G^{N} S S \stackrel{G^{N-1}\left(\partial^{-1}\right)}{\longrightarrow} G^{N-1}(S S) \ldots G(S S) \stackrel{\partial^{-1}}{\longrightarrow} S S$.

Unfolding the definitions yields:

Lemma 5.10. Consider any $u \in U_{d}$, and let $a(u)=\left(z_{1}, \ldots, z_{k}\right)$. For any $f: U \rightarrow S S$, we have

- $\partial^{-1}(G(f)(a(u)))\left(i d_{d}^{\bullet}\right)=k$, and

- $\partial^{-1}(G(f)(a(u)))(b \bullet v)=\sum_{i \in k} f\left(z_{i}(b)\right)(v)$ for any composable basic move $b$ and view $v$.

Corollary 5.11. We have, for any $N \in \mathbb{N}, f_{N}(u)\left(i d_{d}^{\bullet}\right)=k$. Furthermore, for any basic move $b: d^{\prime} \rightarrow d$, and view $v: d^{\prime \prime} \rightarrow d^{\prime}$, we have for any $N \in \mathbb{N}$ :

$$
f_{N+1}(u)(b \bullet v)=\sum_{i \in k} f_{N}\left(z_{i}(b)\right)(v) .
$$

As announced, we have:

Lemma 5.12. For any view $v: d^{\prime} \rightarrow d$ and $n \in \mathbb{N}, f_{|v|+n}(u)(v)=f_{|v|}(u)(v)$.

Proof. We proceed by well-founded induction on $(|v|, n)$, for the lexical ordering. Let again $a(u)=\left(z_{1}, \ldots, z_{k}\right)$. First, we have $f_{\left|i d^{\bullet}\right|}(u)\left(i d^{\bullet}\right)=k$, and for any $n, f_{\mid i d^{\bullet}+n+1}(u)\left(i d^{\bullet}\right)=k$ by Corollary [5.11] Now, if $v=b \bullet v^{\prime}$, then by Corollary 5.11 again:

$$
\begin{aligned}
f_{|v|+n+1}(u)\left(b \bullet v^{\prime}\right) & =\sum_{i \in k} f_{|v|+n}\left(z_{i}(b)\right)\left(v^{\prime}\right) & & \\
& =\sum_{i \in k} f_{\left|v^{\prime}\right|+n+1}\left(z_{i}(b)\right)\left(v^{\prime}\right) & & \text { (by } \left.|v|=\left|v^{\prime}\right|+1\right) \\
& =\sum_{i \in k} f_{\left|v^{\prime}\right|}\left(z_{i}(b)\right)\left(v^{\prime}\right) & & \text { (by induction hypothesis) } \\
& =f_{|v|}(u)\left(b \bullet v^{\prime}\right) & & \text { (by Corollary 5.11 again). }
\end{aligned}
$$


The sequence $\left(f_{n}(u)\right)$ thus has a colimit in $S S_{d}=\widetilde{\mathbb{E}_{d}^{\mathbb{V}}}$ : the presheaf mapping any view $v$ to $f_{|v|}(u)(v)$. This allows us to define:

Definition 5.13. Let $f: U \rightarrow S S$ map any $u \in U_{d}$ to the colimit of the $f_{N}(u)$ 's.

Lemma 5.14. The following diagram commutes:

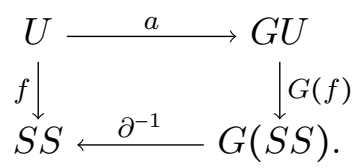

Proof. Consider any $u \in U_{d}$ and view $v$, and let $a(u)=\left(z_{1}, \ldots, z_{k}\right)$. Let also $n=f(u)(v)=$ $f_{|v|}(u)(v)$ and $n^{\prime}=\partial^{-1}(G(f)(a(u)))(v)$.

- If $|v|=0$, then by Lemma $5.10 n=n^{\prime}=k$.

- If $v=b \bullet v^{\prime}$, then by Lemma 5.10 again we have $n^{\prime}=\sum_{i \in k} f\left(z_{i}(b)\right)\left(v^{\prime}\right)$. But by definition of $f$, we obtain $n^{\prime}=\sum_{i \in k} f_{\left|v^{\prime}\right|}\left(z_{i}(b)\right)\left(v^{\prime}\right)$, which is in turn equal to $f_{|v|}(u)(v)=n$ by Corollary 5.11 .

Corollary 5.15. The map $f$ is a map $U \rightarrow S S$ of $G$-coalgebras.

Lemma 5.16. The map $f$ is the unique map $U \rightarrow S S$ of $G$-coalgebras.

Proof. Consider any such map $g$ of coalgebras, and let $a(u)=\left(z_{1}, \ldots, z_{k}\right)$. The map $g$ must be such that

$$
g(u)\left(i d_{d}^{\bullet}\right)=\partial^{-1}(G(g)(a(u)))\left(i d_{d}^{\bullet}\right)=k,
$$

by Lemma 5.10. Furthermore, by the same lemma, it must satisfy:

$$
g(u)(b \bullet v)=\partial^{-1}(G(g)(a(u)))(b \bullet v)=\sum_{i \in k} g\left(z_{i}(b)\right)(v),
$$

which imposes by induction that $f=g$.

The last two results directly entail Theorem 5.7 .

5.2. The labelled transition system for strategies. In this section, we go beyond HP, and define an LTS for strategies, for an arbitrary playground $\mathbb{D}$.

First, the alphabet for our LTS will constist of quasi-moves, in the following sense.

Notation 5.17. We use the following notation for cartesian lifting (by (P1) of a play $u$ along a horizontal morphism $k$ (fixing a global choice of liftings):

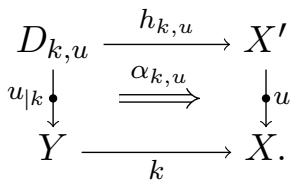

Definition 5.18. A quasi-move is a vertical morphism which locally either is a move or has length 0 . More precisely, a play $u: Y \rightarrow X$ is a quasi-move iff for all players $x: d \rightarrow X$, $u_{\mid x}$ either is a move or has length 0 .

A quasi-move is full when it locally either is a full move or has length 0 . Let $\mathbb{Q}$ denote the subgraph of $\mathbb{D}_{v}$ consisting of full quasi-moves. 
Observe that a quasi-move on an individual either is a move or has length 0 .

States in our LTS will be the following special kind of strategies:

Definition 5.19. A strategy $S \in S S_{X}$ is definite when $\bar{S}\left(i d_{X}^{\bullet}\right)=1$, or equivalently when for all players $(d, x) \in \operatorname{Pl}(X)$, we have $S\left(i d_{d}^{\bullet}, x\right)=1$.

Intuitively, for any quasi-move $X^{\prime} \stackrel{M}{\rightarrow} X$, we would like transitions $\left(X^{\prime}, S^{\prime}\right) \stackrel{M}{\longrightarrow}(X, S)$ in our LTS to occur when $S^{\prime}$ is a definite restriction of $S \cdot M$ to some state of $\bar{S}(M)$. I.e., a transition roughly corresponds to a way for $\bar{S}$ to accept $M$. However, $S \cdot M$ is not quite $\bar{S}(M)$ so the right notion of restriction may not be obvious. But we have defined a notion of restriction in Definition 5.5, for strategies on individuals. We now define restriction for general strategies, and use this to define our LTS. Finally, we elucidate the connection with $\bar{S}(M)$.

Consider, for any $S \in S S_{X}$ and $\sigma \in \prod_{(d, x) \in \operatorname{Pl}(X)} S\left(i d_{d}^{\bullet}, x\right)$, and recall from below Definition 4.33 that $S \cdot h$ is shorthand for the image of $S \in S S_{X}$ under the action of a horizontal morphism $h: Y \rightarrow X$ for the horizontal part of our pseudo double functor $S S$.

Definition 5.20. Let the restriction $S_{\mid \sigma} \in S S_{X}$ of $S$ to $\sigma$ be defined by the fact that for any player $x: d \rightarrow X$,

$$
\left(S_{\mid \sigma}\right) \cdot x=(S \cdot x)_{\mid \sigma(d, x)} .
$$

Concretely, we have, for any $v, S_{\mid \sigma}(v, x)=\left\{\sigma^{\prime} \in S(v, x) \mid S\left(!_{v}\right)\left(\sigma^{\prime}\right)=\sigma(d, x)\right\}$, where $!_{v}$ is the unique morphism $\left(i d_{d}^{\bullet}, x\right) \rightarrow(v, x)$ in $\mathbb{E}_{X}^{\mathbb{V}}$.

We now define our LTS for strategies over $\mathbb{Q}$.

Definition 5.21. The underlying graph $\mathcal{S}_{\mathbb{D}}$ for our LTS is the graph with as vertices all pairs $(X, S)$ where $X$ is a position and $S \in S S_{X}$ is a definite strategy, and whose edges $\left(X^{\prime}, S^{\prime}\right) \rightarrow(X, S)$ are all full quasi-moves $M: X^{\prime} \rightarrow X$ such that there exists a state $\sigma \epsilon$ $\prod_{\left(d^{\prime}, x^{\prime}\right) \in \operatorname{Pl}\left(X^{\prime}\right)}(S \cdot M)\left(i d_{d^{\prime}}^{\bullet}, x^{\prime}\right)$ with $S^{\prime}=(S \cdot M)_{\mid \sigma}$.

The assignment $(X, S) \mapsto X$ defines a morphism $p_{S S}: \mathcal{S}_{\mathbb{D}} \rightarrow \mathbb{Q}$ of reflexive graphs, which is our LTS.

An alternative characterisation of transitions $(X, S) \stackrel{M}{\longleftarrow}\left(X^{\prime}, S^{\prime}\right)$ is the existence of $\sigma$ such that

for all $\left(d^{\prime}, x^{\prime}\right) \in \operatorname{Pl}\left(X^{\prime}\right)$.

$$
S^{\prime} \cdot x^{\prime}=\left(S \cdot M \cdot x^{\prime}\right)_{\mid \sigma\left(d^{\prime}, x^{\prime}\right)}=\left(S \cdot\left(x^{\prime}\right)^{M} \cdot v^{x^{\prime}, M}\right)_{\mid \sigma\left(d^{\prime}, x^{\prime}\right)}
$$

Let us now return to the connection between $\prod_{\left(d^{\prime}, x^{\prime}\right) \in \operatorname{Pl}\left(X^{\prime}\right)}(S \cdot M)\left(i d_{d^{\prime}}^{\bullet}, x^{\prime}\right)$ and $\bar{S}(M)$. First, we have by definition $(S \cdot M)\left(i d_{d^{\prime}}^{\bullet}, x^{\prime}\right)=S\left(v^{x^{\prime}, M},\left(x^{\prime}\right)^{M}\right)$, for any player $x^{\prime}: d^{\prime} \rightarrow X^{\prime}$. Now, as recalled above, $\bar{S}(M)$ may be characterised as a limit of

$$
\left(\mathbb{E}_{X}^{\mathbb{V}} / M\right)^{\text {op }} \stackrel{\text { dom }}{\longrightarrow}\left(\mathbb{E}_{X}^{\mathbb{V}}\right)^{\text {op }} \stackrel{S}{\rightarrow} \text { ford } \hookrightarrow \text { set. }
$$

Since $\alpha^{x^{\prime}, M}: v^{x^{\prime}, M} \rightarrow M$ is an object in $\left(\mathbb{E}_{X}^{\mathbb{V}} / M\right)^{o p}$, we obtain by projection a map $\bar{S}(M) \rightarrow$ $S\left(v^{x^{\prime}, M},\left(x^{\prime}\right)^{M}\right)$.

Definition 5.22. For any $S \in S S_{X}$, let $\psi_{M}: \bar{S}(M) \rightarrow \prod_{\left(d^{\prime}, x^{\prime}\right) \in \operatorname{Pl}\left(X^{\prime}\right)} S\left(v^{x^{\prime}, M},\left(x^{\prime}\right)^{M}\right)$ denote the corresponding tupling map.

Proposition 5.23. For any definite $S \in S S_{X}$, the map $\psi_{M}$ is a bijection. 
We prove this through the following lemma. For any full quasi-move $M: X^{\prime} \rightarrow X$, observe that for any player $x^{\prime}: d^{\prime} \rightarrow X^{\prime}, v^{x^{\prime}, M}$ has length at most 1 (consider $M_{\mid\left(x^{\prime}\right)^{M}}$ ), and let

$$
\mathrm{Pl}_{M}\left(X^{\prime}\right)=\left\{\left(d^{\prime}, x^{\prime}\right) \in \operatorname{Pl}\left(X^{\prime}\right)|| v^{x^{\prime}, M} \mid \neq 0\right\} .
$$

Lemma 5.24. For any definite $S \in S S_{X}$, and full quasi-move $M: X^{\prime} \rightarrow X$, the map

$$
\bar{S}(M) \stackrel{\psi_{M}}{\longrightarrow} \prod_{\left(d^{\prime}, x^{\prime}\right) \in \operatorname{Pl}\left(X^{\prime}\right)} S\left(v^{x^{\prime}, M},\left(x^{\prime}\right)^{M}\right) \rightarrow \prod_{\left(d^{\prime}, x^{\prime}\right) \in \mathrm{Pl}_{M}\left(X^{\prime}\right)} S\left(v^{x^{\prime}, M},\left(x^{\prime}\right)^{M}\right),
$$

where the second map is by projection, is bijective.

Proof. Recall that $\bar{S}(M)$ is a limit of

$$
\left(\mathbb{E}_{X}^{\mathbb{V}} / M\right)^{o p} \stackrel{\text { dom }}{\longrightarrow}\left(\mathbb{E}_{X}^{\mathbb{V}}\right)^{o p} \stackrel{S}{\rightarrow} \text { ford } \hookrightarrow \text { set },
$$

and consider the poset $P$ with underlying set $\mathrm{Pl}(X)+\mathrm{Pl}_{M}\left(X^{\prime}\right)$ and ordering given by $(d, x)<$ $\left(d^{\prime}, x^{\prime}\right)$ iff $x=\left(x^{\prime}\right)^{M}$. Consider the functor $p: P \rightarrow \mathbb{E}_{X}^{\mathbb{V}} / M$ mapping any $(d, x) \in \operatorname{Pl}(X)$ to the unique morphism $i d_{d}^{\bullet} \rightarrow M$ with lower border $x$, and any $\left(d^{\prime}, x^{\prime}\right) \in \mathrm{Pl}_{M}\left(X^{\prime}\right)$ to $\alpha^{x^{\prime}, M}$. Since $P$ is a poset, $p$ is faithful. It is furthermore full by Proposition 4.27, Finally, for any $(w, \alpha): v \rightarrow M$ in $\mathbb{E}_{X}^{\mathbb{V}} / M$,

- either $|v|=0$ and there is a unique player $x: d \rightarrow X$ such that $(w, \alpha)$ is the (unique) morphism $i d_{d}^{\bullet} \rightarrow M$ with lower border $x$,

- or $|v|=1$ and there exists a unique player $\left(d^{\prime}, x^{\prime}\right) \in X^{\prime}$ such that $(w, \alpha)=\left(i d, \alpha^{x^{\prime}, M}\right)$ (let $x=\operatorname{cod}(\alpha) ;\left|M_{\mid x}\right|=1$, so by Proposition $4.25|w|=0$ ).

This entails that $p$ is essentially surjective on objects, hence an equivalence. Thus, $\bar{S}(M)$ is also a limit of

$$
P^{o p} \simeq\left(\mathbb{E}_{X}^{\mathbb{V}} / M\right)^{o p} \stackrel{\text { dom }}{\longrightarrow}\left(\mathbb{E}_{X}^{\mathbb{V}}\right)^{o p} \stackrel{S}{\rightarrow} \text { ford } \hookrightarrow \text { set. }
$$

But now, because $S$ is definite, this functor maps any $(d, x) \in \operatorname{Pl}(X)$ to a singleton, hence $\bar{S}(M)$ is also a limit of

$$
\mathrm{Pl}_{M}\left(X^{\prime}\right) \hookrightarrow P^{o p} \simeq\left(\mathbb{E}_{X}^{\mathbb{V}} / M\right)^{o p} \stackrel{\text { dom }}{\longrightarrow}\left(\mathbb{E}_{X}^{\mathbb{V}}\right)^{o p} \stackrel{S}{\rightarrow} \text { ford } \hookrightarrow \text { set },
$$

i.e., isomorphic to $\prod_{\left(d^{\prime}, x^{\prime}\right) \in \mathrm{Pl}_{M}\left(X^{\prime}\right)} S\left(v^{x^{\prime}, M},\left(x^{\prime}\right)^{M}\right)$, as desired.

Proof of Proposition 5.23. If $\left|v^{x^{\prime}, M}\right|=0$, then $S\left(v^{x^{\prime}, M},\left(x^{\prime}\right)^{M}\right)$ is a singleton. Thus, the second map of Lemma 5.24 is bijective, hence so is $\psi_{M}$.

The moral of Proposition 5.23 is that transitions $(X, S) \stackrel{M}{\longleftarrow}\left(X^{\prime}, S^{\prime}\right)$ in $\mathcal{S}_{\mathbb{D}}$ are precisely given by full quasi-moves $M: X^{\prime} \rightarrow X$ such that there exists a state $\sigma \in \bar{S}(M)$ with

$$
S^{\prime}=(S \cdot M)_{\mid \psi_{M}(\sigma)},
$$

for all $\left(d^{\prime}, x^{\prime}\right) \in \operatorname{Pl}\left(X^{\prime}\right)$.

We now give more syntactic characterisations of transitions, starting with transitions from states of the shape $(d, S)$. Recall the syntax for strategies below Theorem 5.7 .

Proposition 5.25. If $S=\left\langle\left(S_{b}\right)_{b \in[\mathbb{B}]_{d}}\right\rangle$ is a definite strategy on $d \in \mathbb{I}$, and if for all $b \in[\mathbb{B}]_{d}$, $S_{b}=\bigoplus_{i \in n_{b}} D_{i}^{b}$ for definite $D_{i}^{b}$, then for any $M: X^{\prime} \rightarrow d$ we have $(d, S) \stackrel{M}{\longleftarrow}\left(X^{\prime}, S^{\prime}\right)$ iff

- for all $\left(d^{\prime}, x^{\prime}\right) \in \mathrm{Pl}_{M}\left(X^{\prime}\right)$, there exists $i_{x^{\prime}} \in n_{v^{x^{\prime}, M}}$ such that $S^{\prime} \cdot x^{\prime}=D_{i_{x^{\prime}}^{v^{x^{\prime}, M}}}$, 
- and for all $\left(d^{\prime}, x^{\prime}\right) \in \mathrm{Pl}\left(X^{\prime}\right) \backslash \mathrm{Pl}_{M}\left(X^{\prime}\right), S^{\prime} \cdot x^{\prime}=S$.

Let us now characterise transitions from arbitrary positions in terms of their restrictions to individuals. Recalling Notation [5.17, we have:

Proposition 5.26. We have $(X, S) \stackrel{M}{\longleftarrow}\left(X^{\prime}, S^{\prime}\right)$ iff for all $(d, x) \in \operatorname{Pl}(X)$,

$$
(d, S \cdot x) \stackrel{M_{\mid x}}{\longleftarrow}\left(D_{x, M}, S^{\prime} \cdot h_{x, M}\right) .
$$

Putting both previous results together, we obtain:

Corollary 5.27. Let, for all $(d, x) \in \operatorname{Pl}(X), S \cdot x=\left\langle\left(S_{b}^{x}\right)_{b \in[\mathbb{B}]_{d}}\right\rangle$ and for all $b \in[\mathbb{B}]_{d}$, $S_{b}^{x}=\bigoplus_{i \in n_{b}^{x}} D_{i}^{x, b}$ for definite $D_{i}^{x, b}$.

Then, for any $M: X^{\prime} \rightarrow X$, we have $(X, S) \stackrel{M}{\longleftarrow}\left(X^{\prime}, S^{\prime}\right)$ iff

- for all $\left(d^{\prime}, x^{\prime}\right) \in \mathrm{Pl}_{M}\left(X^{\prime}\right)$, there exists $i_{x^{\prime}} \in n_{v_{x^{\prime}, M}}^{\left(x^{\prime}\right)^{M}}$ such that $S^{\prime} \cdot x^{\prime}=D_{i_{x^{\prime}}}^{\left(x^{\prime}\right)^{M}, v^{x^{\prime}, M}}$,

- and for all $\left(d^{\prime}, x^{\prime}\right) \in \operatorname{Pl}\left(X^{\prime}\right) \backslash \mathrm{Pl}_{M}\left(X^{\prime}\right), S^{\prime} \cdot x^{\prime}=S \cdot\left(x^{\prime}\right)^{M}$.

5.3. Process terms. In the previous section, starting from a playground $\mathbb{D}$, we have constructed an LTS $\mathcal{S}_{\mathbb{D}}$ of strategies. We now begin the construction of the LTS $\mathcal{T}_{\mathbb{D}}$ of process terms announced in Section 1.3. starting with process terms themselves.

Definition 5.28. For any $X$, let $[\mathbb{F}]_{X}$ be the set of isomorphism classes of full moves with codomain $X$, in $D(X)$, and let $\chi$ denote the map

$$
\begin{aligned}
{[\mathbb{F}]_{d} } & \rightarrow \mathcal{P}_{f}\left([\mathbb{B}]_{d}\right) \\
M & \mapsto\left\{[b] \in[\mathbb{B}]_{d} \mid \exists \alpha \in D(b, M)\right\} .
\end{aligned}
$$

Let $\left[\mathbb{F}^{1}\right]_{d}$ denote the subset of $[\mathbb{F}]_{d}$ consisting of (isomorphism classes of) full moves $M: X^{\prime} \rightarrow d$ such that $\mathrm{Pl}_{M}\left(X^{\prime}\right)$ is a singleton (and hence so is $\chi(M)$ ). Let $\left[\mathbb{F}^{+}\right]_{d}$ denote the complement subset.

The map $\chi$ is easily checked to be well-defined.

We state one more axiom to demand that basic sub-moves of a full move $[M] \in[\mathbb{F}]_{d}$ may not be sub-moves of other full moves.

Axiom. (P10) (Basic vs. full) For any $d \in \mathbb{I}$ and $M, M^{\prime} \in[\mathbb{F}]_{d}$, if $M \neq M^{\prime}$, then $\chi(M) \cap$ $\chi\left(M^{\prime}\right)=\varnothing$.

Let process terms be infinite terms in the typed grammar:

$$
\begin{gathered}
\frac{\ldots d_{i} \vdash T_{i} \ldots(\forall i \in n)}{d \vdash \sum_{i \in n} M_{i} \cdot T_{i}}\left(n \in \mathbb{N} ; \forall i \in n, M_{i} \in\left[\mathbb{F}^{1}\right]_{d} \text { and } \chi\left[M_{i}\right]=\left\{b_{i}: d_{i} \rightarrow d\right\}\right) \\
\frac{\ldots d^{\prime} \vdash T_{b} \ldots\left(\forall\left(b: d^{\prime} \rightarrow d\right) \in \chi[M]\right)}{d \vdash M\left\langle\left(T_{b}\right)_{b \in \chi[M]}\right\rangle}\left(M \in\left[\mathbb{F}^{+}\right]_{d}\right) .
\end{gathered}
$$

The first rule is a guarded sum, in a sense analogous to guarded sum in CCS. It should be noted that guards have to be full moves with only one non-trivial view. There is good reason for that, since allowing general moves as guards would break bisimilarity between process terms and strategies. To understand this, consider a hypothetical guarded sum 
$R=(P \mid Q)+\left(P^{\prime} \mid Q^{\prime}\right)$. Since this has no interaction before the choice is made, $R$ behaves, in CCS, just like an internal choice $(P \mid Q) \oplus\left(P^{\prime} \mid Q^{\prime}\right)$. However, our translation to strategies does not translate guarded sum as an internal choice, with right, since other guarded sums, e.g., a. $P+b . Q$ should certainly not be translated this way. Instead, $R$ would be translated as something equivalent to $(P \mid Q) \oplus\left(P^{\prime} \mid Q\right) \oplus\left(P \mid Q^{\prime}\right) \oplus\left(P^{\prime} \mid Q^{\prime}\right)$, which is clearly not bisimilar to $R$ in general.

We could easily include internal choice in the grammar, since strategies do model it, directly. We refrain from doing so for simplicity.

Definition 5.29. Let $T_{\mathbb{D}}$ be the set of process terms.

Example 5.30. For $\mathbb{D}^{C C S}$, the obtained syntax is equivalent to

$$
\frac{\ldots \Gamma \cdot \alpha_{i} \vdash P_{i} \ldots}{\Gamma \vdash \sum_{i} \alpha_{i} \cdot P_{i}} \quad \frac{\Gamma \vdash P \quad \Gamma \vdash Q}{\Gamma \vdash P \mid Q} .
$$

where

- $\Gamma$ ranges over natural numbers;

- $\alpha::=a|\bar{a}| \nabla \mid \nu($ for $a \in \Gamma)$;

- $\Gamma \cdot \alpha$ denotes $(\Gamma+1)$ if $\alpha=\nu$ and just $\Gamma$ otherwise.

This grammar obviously contains CCS, and we let $\theta: \mathrm{ob}(C C S) \hookrightarrow \mathrm{T}_{\mathbb{D} C C S}$ be the injection.

5.4. The labelled transition system for process terms. We now define the LTS $\mathcal{T}_{\mathbb{D}}$. States, i.e., vertices of the graph underlying this LTS, are pairs $(X, T)$ of a position $X$ and a family $T$ of process terms, indexed by the players of $X$, i.e., $T \in \prod_{(d, x) \in \operatorname{Pl}(X)}\left(\mathrm{T}_{\mathbb{D}}\right)_{d}$, where $\left(\mathrm{T}_{\mathbb{D}}\right)_{d}$ is the set of process terms of type $d$.

To define edges, we need a lemma. For any play $u: X^{\prime} \rightarrow X$ and $x: d \rightarrow X$, recalling Notation 5.17, consider the map

$$
\begin{aligned}
r^{u}: \quad \sum_{(d, x) \in \operatorname{Pl}(X)} \operatorname{Pl}\left(D_{x, u}\right) & \rightarrow \operatorname{Pl}\left(X^{\prime}\right) \\
\left((d, x),\left(d^{\prime}, x^{\prime}\right)\right) & \mapsto h_{x, u} \circ x^{\prime}
\end{aligned}
$$

sending any $(d, x) \in \operatorname{Pl}(X)$ and $x^{\prime}: d^{\prime} \rightarrow D_{x, u}$ to $d^{\prime} \stackrel{x^{\prime}}{\rightarrow} D_{x, u} \stackrel{h_{x, u}}{\longrightarrow} X^{\prime}$.

Consider also the map $i^{u}$ in the other direction sending any $y: d^{\prime} \rightarrow X^{\prime}$ to the pair $\left(\left(d^{y, u}, y^{u}\right),\left(d^{\prime}, y_{\mid y^{u}}\right)\right)$, where $y_{\mid y^{u}}$ is the (domain in $\mathbb{D}_{V}$ of the) unique $\alpha^{\prime}$ making the diagram

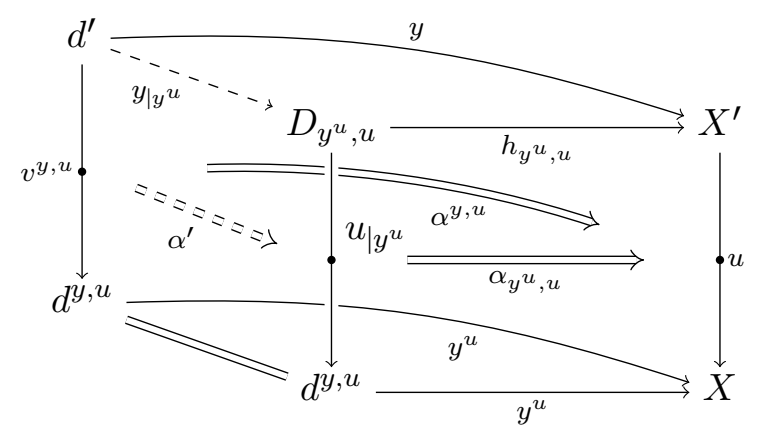

commute (by (P1)). This map $i^{u}$ is well-defined by uniqueness of $y^{u}$ and cartesianness of $\alpha_{y^{u}, u}$. 
Lemma 5.31. The maps $i^{u}$ are $r^{u}$ are mutually inverse.

Proof. Straightforward.

Let us return to the definition of our LTS. We first say that for any full quasi-move $M: D \rightarrow d$, a process term $d \vdash T$ has an $M$-transition to $\left(D, T^{\prime}\right)$, for $T^{\prime} \in \prod_{\left(d^{\prime}, x^{\prime}\right) \in \operatorname{Pl}(D)}\left(\mathrm{T}_{\mathbb{D}}\right)_{d^{\prime}}$, when one of the following holds:

(i) $\exists M^{\prime} \in\left[\mathbb{F}^{+}\right], T=M^{\prime}\left\langle T^{\prime \prime}\right\rangle$, and, for all $\left(d^{\prime}, x^{\prime}\right) \in \operatorname{Pl}(D)$,

- if $v^{x^{\prime}, M}$ is a basic move, then $v^{x^{\prime}, M} \in \chi\left(M^{\prime}\right)$ and $T_{d^{\prime}, x^{\prime}}^{\prime}=T_{v^{x^{\prime}, M}}^{\prime \prime}$;

- otherwise $\left|v^{x^{\prime}, M}\right|=0$ (hence $d^{\prime}=d$ ), and $T_{d^{\prime}, x^{\prime}}^{\prime}=T$;

(ii) $[M] \in\left[\mathbb{F}^{1}\right], T=\sum_{i \in n} M_{i} \cdot T_{i}, M_{i_{0}}=[M]$ for some $i_{0} \in n$, and for all players $x^{\prime}: d^{\prime} \rightarrow D$ - if $v^{x^{\prime}, M} \in \chi(M)$, then $T_{d^{\prime}, x^{\prime}}^{\prime}=T_{i_{0}}$,

- and otherwise $\left(\left|v^{x^{\prime}, M}\right|=0\right), T_{d^{\prime}, x^{\prime}}^{\prime}=T$;

(iii) $|M|=0$ and for all $\left(d^{\prime}, x^{\prime}\right) \in \operatorname{Pl}(D), T_{d^{\prime}, x^{\prime}}^{\prime}=T$ (which, again, makes sense by Lemma 4.17).

We denote such a transition by $T \stackrel{M}{\longleftarrow}\left(D, T^{\prime}\right)$.

Remark 5.32. The first case $(i)$ allows $\chi(M)=\varnothing$, but if $\chi(M) \neq \varnothing$, then $[M]=M^{\prime}$ by (P10), Also, let us mention that $\chi(M) \neq \varnothing$ does not imply $|M|=0$ in general, although it does in $\mathbb{D}^{C C S}$.

Definition 5.33. Let $\mathcal{T}_{\mathbb{D}}$ be the graph with pairs $(X, T)$ as vertices, and as edges $\left(X^{\prime}, T^{\prime}\right) \rightarrow$ $(X, T)$ full quasi-moves $M: X^{\prime} \rightarrow X$ such that for all $(d, x) \in \operatorname{Pl}(X), T_{d, x} \stackrel{M_{x x}}{\longleftarrow}\left(D_{x, M},\left(T^{\prime} \circ\right.\right.$ $\left.\left.\Sigma_{h_{x, M}}\right)\right)$. Here, we let $\Sigma_{h_{x, M}}$ denote composition with $h_{x, M}: D_{x, M} \rightarrow X^{\prime}$, viewed as a map $\operatorname{Pl}\left(D_{x, M}\right) \rightarrow \operatorname{Pl}\left(X^{\prime}\right)$.

$\mathcal{T}_{\mathbb{D}}$ is viewed as an LTS over $\mathbb{Q}$, by mapping $(X, T) \stackrel{M}{\longleftarrow}\left(X^{\prime}, T^{\prime}\right)$ to $X \stackrel{M}{\bullet} X^{\prime}$.

Example 5.34. For $\mathbb{D}^{C C S}$, the obtained LTS differs subtly, but significantly from the usual LTS for CCS. In order to explain this clearly, let us introduce some notation. First, let evaluation contexts be generated by the grammar

$$
\overline{\Gamma ; x: n \vdash x\left(a_{1}, \ldots, a_{n}\right)} \quad \frac{\Gamma ; \Delta_{1} \vdash \mathrm{e}_{1} \quad \Gamma ; \Delta_{2} \vdash \mathrm{e}_{2}}{\Gamma ; \Delta_{1}, \Delta_{2} \vdash \mathrm{e}_{1} \mid \mathrm{e}_{2}},
$$

where, in the first rule, $\forall i \in n, a_{i} \in \Gamma$, and in the second $\operatorname{dom}\left(\Delta_{1}\right) \cap \operatorname{dom}\left(\Delta_{2}\right)=\varnothing$. Here, $x$ ranges over a fixed set of variables, and $\Delta, \ldots$ range over finite maps from variables to natural numbers. Evaluation contexts are considered equivalent up to associativity and commutativity of $\mid$. Positions are essentially a combinatorial, direct representation of such contexts.

Leaving the details aside, states in $\mathcal{T}_{\mathbb{D} C C S}$ may be viewed as pairs $(X, T)$ of an evaluation context $X$, plus, for each $n$-ary variable $x\left(a_{1}, \ldots, a_{n}\right)$ in $X$, a process term over $n$ in the grammar of Example 5.30. Instead of separately writing the evaluation context and the map from its variables to process terms, we inline process terms between brackets in the context, thus avoiding variables. Moves are either put in context similarly, or located implicitly. E.g., for a state $(X, T)$ where $X$ contains two players respectively mapped by $T$ to process terms $P$ and $Q$, we would write $[P] \mid[Q]$. There is some ambiguity in this notation, e.g., in case some channels are absent from $P$ : are they absent from the arity of $P$, or only unused? Since we use this notation mostly for clarifying examples, we will avoid such ambiguities. 
Finally, we sometimes use brackets to denote the fact that some holes are filled with the given state. E.g., $X[[P] \mid[Q]]$ denotes a state $X$, where a hole has been replaced by a parallel composition of two holes, respectively filled with $P$ and $Q$.

Returning to our comparison of $\mathcal{T}_{\mathbb{D} C C S}$ and $C C S$, of course, a first difference is the fact that labels may contain several moves, as quasi-moves only locally have length 1.

A second difference is the presence of heating rules for parallel composition and channel creation, in a sense close to the chemical abstract machine [4]. For example, we have transitions $X[P \mid Q] \stackrel{\pi}{\leftarrow} X[[P] \mid[Q]]$.

There is a third important difference, related to channel creation. For instance, we have transitions

$$
[\text { va.a.P }] \stackrel{\nu}{\leftarrow}\left[\text { a.P } \stackrel{\iota_{a}}{\longleftarrow}[P] .\right.
$$

The second transition cannot occur in a closed-world setting, since the environment cannot know $a$. And it does not occur in $C C S$ either.

A final difference is that labels contain too much information to be relevant for behavioural equivalences. E.g., they contain the whole evaluation context in which the transition takes place, as well as which players are involved.

The second difference, i.e., the presence of heating rules, is not really problematic, and merely forces us to use weak bisimulations rather than strong ones. All other defects will be corrected below.

5.5. Translation and a first correctness result. We conclude this section on the general theory of playgrounds by establishing a strong, functional bisimulation from process terms to strategies.

Mimicking (3.2) (page 23), our translation from process terms to definite strategies (qua families over $\mathbb{I}$ ) is defined coinductively by

$$
\begin{aligned}
\llbracket \sum_{i \in n} M_{i} . T_{i} \rrbracket & \left.=\left\langle b \mapsto \bigoplus_{\{i \in n \mid b \in \chi(}\left(M_{i}\right)\right\} \llbracket T_{i} \rrbracket\right\rangle \\
\llbracket M\left\langle\left(T_{b}\right)_{b \in \chi(M)}\right\rangle \rrbracket & =\left\langle b \mapsto\left\{\begin{array}{ll}
\llbracket T_{b} \rrbracket & \text { if } b \in \chi(M) \\
\varnothing & \text { otherwise }
\end{array}\right\rangle .\right.
\end{aligned}
$$

Let us extend the map $\llbracket-\rrbracket: \mathrm{T}_{\mathbb{D}} \rightarrow S S_{\mathbb{D}}$ to a map $\llbracket-\rrbracket: \mathrm{ob}\left(\mathcal{T}_{\mathbb{D}}\right) \rightarrow \mathrm{ob}\left(\mathcal{S}_{\mathbb{D}}\right)$, defined by $\llbracket X, T \rrbracket=\left(X,\left(\llbracket T_{d, x} \rrbracket\right)_{(d, x) \in \operatorname{Pl}(X)}\right)$, using Proposition 5.1

Theorem 5.35. The map $\llbracket-\rrbracket: \mathrm{ob}\left(\mathcal{T}_{\mathbb{D}}\right) \rightarrow \mathrm{ob}\left(\mathcal{S}_{\mathbb{D}}\right)$ is a functional, strong bisimulation.

Proof. The theorem follows from Proposition 5.26 and the next lemma.

Lemma 5.36. For any full quasi-move $M: X^{\prime} \rightarrow d$, for any $T \in\left(\mathrm{T}_{\mathbb{D}}\right)_{d}$ and $S^{\prime} \in\left(S S_{\mathbb{D}}\right)_{X^{\prime}}$, we have

$$
(d, \llbracket T \rrbracket) \stackrel{M}{\longleftarrow}\left(X^{\prime}, S^{\prime}\right) \quad \text { iff } \quad \exists T^{\prime},\left(T \stackrel{M}{\longleftarrow}\left(X^{\prime}, T^{\prime}\right)\right) \wedge\left(\left(X^{\prime}, S^{\prime}\right)=\llbracket X^{\prime}, T^{\prime} \rrbracket\right) .
$$

Note the implicit typing: $T^{\prime} \in \prod_{\left(d^{\prime}, x^{\prime}\right) \in \operatorname{Pl}\left(X^{\prime}\right)}\left(\mathrm{T}_{\mathbb{D}}\right)_{d^{\prime}}$. Also the second condition on the right is equivalent to $\forall x^{\prime}: d^{\prime} \rightarrow X^{\prime}, S^{\prime} \cdot x^{\prime}=\llbracket T_{d^{\prime}, x^{\prime}}^{\prime} \rrbracket$.

Proof. If $|M|=0$, then both sides are equivalent to the fact that for all $x^{\prime}: d^{\prime} \rightarrow X^{\prime}, S^{\prime} \cdot x^{\prime}=$ $\llbracket T \rrbracket$.

Otherwise, we proceed by case analysis on $T$.

If $T=M^{\prime}\left\langle\left(T_{b}^{\prime \prime}\right)_{b \in \chi\left(M^{\prime}\right)}\right\rangle$, then by (P10) both sides are equivalent to $\chi(M) \subseteq \chi\left(M^{\prime}\right)$, plus

- for all $\left(d^{\prime}, x^{\prime}\right) \in \mathrm{Pl}_{M}\left(X^{\prime}\right), S^{\prime} \cdot x^{\prime}=\llbracket T_{v^{x^{\prime}, M}}^{\prime \prime} \rrbracket$, and 
- for all $\left(d^{\prime}, x^{\prime}\right) \in \mathrm{Pl}\left(X^{\prime}\right) \backslash \mathrm{Pl}_{M}\left(X^{\prime}\right), S^{\prime} \cdot x^{\prime}=\llbracket T \rrbracket$.

Indeed, for any $b \in \chi(M), \llbracket T \rrbracket \cdot b=\llbracket T_{b}^{\prime \prime} \rrbracket$ is definite. We thus put $T_{d^{\prime}, x^{\prime}}^{\prime}=T_{v^{x^{\prime}, M}}^{\prime \prime}$ in the first case and $T_{d^{\prime}, x^{\prime}}^{\prime}=T$ in the second case.

If $T=\sum_{i \in n} M_{i} \cdot T_{i}$, then both sides are equivalent to the existence of $i_{0} \in n$ such that $M_{i_{0}}=[M]$ and

- for the unique $\left(d^{\prime}, x^{\prime}\right) \in \mathrm{Pl}_{M}\left(X^{\prime}\right), S^{\prime} \cdot x^{\prime}=\llbracket T_{i_{0}} \rrbracket$, and

- for all $\left(d^{\prime}, x^{\prime}\right) \in \operatorname{Pl}\left(X^{\prime}\right) \backslash \mathrm{Pl}_{M}\left(X^{\prime}\right), S^{\prime} \cdot x^{\prime}=\llbracket T \rrbracket$.

This uses (P10), since the left-hand side unfolds to the existence of $x^{\prime}: d^{\prime} \rightarrow X^{\prime}$ such that $v^{x^{\prime}, M} \in \chi[\bar{M}]$ and $\llbracket T \rrbracket \cdot v^{x^{\prime}, M} \neq \varnothing$, i.e., $v^{x^{\prime}, M} \in \chi\left(M_{i_{0}}\right)$ for some $i_{0} \in n$, by definition of $\llbracket T \rrbracket$. This entails in particular $[M]=M_{i_{0}}$ by $(\mathrm{P} 10)$,

\section{GRAPHS AND FAIR MORPHISMS}

In this section, we derive our main result. For this, we develop a notion of graph with complementarity, which aims at being a theory of LTSs over which fair testing makes sense. Although the theory would apply with any predicate $\perp$ compatible with $\approx_{\Sigma}$ equivalence classes (see below), the question of whether such a generalisation would have useful applications is deferred for now.

For any graph with complementarity $A$ and relation $R: G \longrightarrow H$ over $A$, we exhibit sufficient conditions for $R$ to be fair, i.e., to preserve and reflect fair testing equivalence. We then relate this theory to our semantics, and show that it entails our main result. For now, this section lies outside the scope of playground theory. Some aspects of it could be formalised there, but we leave the complete formalisation for further work. Because the only playground involved is $\mathbb{D}^{C C S}$, we often omit sub or superscripts, e.g., in $\mathbb{D}, \mathcal{S}_{\mathbb{D}}$ (even just $\mathcal{S})$, etc.

Before we start, let us define $\mathbb{W}_{C C S}$ to be the set of closed-world quasi-moves, i.e., vertical morphisms in $\mathbb{D}$ which either are closed-world moves (Definition 3.22) or have length 0 . Please note: quasi-moves must locally restrict to plays of length $\leq 1$, whereas closed-world quasi-moves have length $\leq 1$ globally. Let $\mathbb{D}^{\mathbb{W}}$ be the subbicategory of $\mathbb{D}_{v}$ generated by $\mathbb{W}_{C C S}$, and let $\Sigma$ be the free reflexive graph on an endo-edge $\odot$. Finally, let $\bar{\ell}_{\mathbb{D}}: \mathbb{D}^{\mathbb{W}} \rightarrow \mathrm{fc}(\Sigma)$ be the pseudo functor determined by the mapping $\ell_{\mathbb{D}}: \mathbb{W}_{C C S} \rightarrow \Sigma$ sending all closed-world quasi-moves to $i d$ except $\odot$ moves, which are sent to $\nabla$.

6.1. Graphs with complementarity. A relation $A \mapsto B$ between two reflexive graphs $A$ and $B$ is a subgraph $R \hookrightarrow A \times B$. Such a relation $R$ is total when, for all vertices, resp. edges, $x \in A$, there exists a vertex, resp. an edge $y \in B$, such that $(x, y) \in R$. It is partially functional if there is at most one such $y$. It is functional when it is total and partially functional. The domain of $R$ is the subgraph of $A$ consisting of vertices and edges related to something in $B$.

Definition 6.1. A graph with complementarity is a reflexive graph $A$, equipped with a subgraph $A^{\mathbb{W}}$, a relation $\triangleright^{A}: A^{2} \rightarrow A^{\mathbb{W}}$, and a map $\ell^{A}: A^{\mathbb{W}} \rightarrow \Sigma$, such that the composite $A^{2} \longrightarrow A^{\mathbb{W}} \rightarrow \Sigma$ is partially functional and symmetric.

We let $A^{\complement}=\operatorname{dom}\left(\triangleright^{A}\right)$ and write $a \smile a^{\prime}$ for $\left(a, a^{\prime}\right) \in A^{\complement}$. We further denote the map $A^{\complement} \hookrightarrow A^{2} \longrightarrow A^{\mathbb{W}} \rightarrow \Sigma$ by $(a, b) \mapsto(a \Downarrow b)$, and deem edges in $A^{\mathbb{W}}$ closed-world. 
Remark 6.2. $A^{\complement}$ has to be symmetric as the domain of a symmetric relation.

Definition 6.3. A morphism of graphs with complementarity is a morphism $f: A \rightarrow B$ of reflexive graphs such that

$$
f\left(A^{\mathbb{W}}\right) \subseteq B^{\mathbb{W}} \quad \ell^{B} \circ f^{\mathbb{W}}=\ell^{A} \quad\left(\left(a_{1}, a_{2}\right) \triangleright^{A} a_{3}\right) \Rightarrow\left(\left(f\left(a_{1}\right), f\left(a_{2}\right)\right) \triangleright^{A} f\left(a_{3}\right)\right),
$$

where $f^{\mathbb{W}}: A^{\mathbb{W}} \rightarrow B^{\mathbb{W}}$ is the restriction of $f$.

Proposition 6.4. Graphs with complementarity and morphisms between them form a category GCompl.

We now introduce the graph $\mathbb{I} \mathbb{Q}$, which as announced in the introduction will serve as a base for making $\mathcal{S}_{\mathbb{D} C C S}$ and $\mathcal{T}_{\mathbb{D} C C S}$ into graphs with complementarity. It is an interfaced variant of $\mathbb{Q}$, hence its name.

Example 6.5. Let $\mathbb{I} \mathbb{Q}$ be the graph with as vertices all horizontal morphisms $h: I \rightarrow X$ from some interface to some position, and whose edges $k \rightarrow h$ are given by diagrams

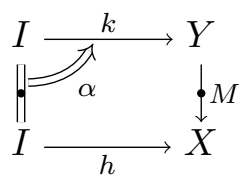

in $D$, where $M$ is either a full move or an identity, such that if $M$ is an input or an output, then the corresponding channel is in the image of $I . \mathbb{I} \mathbb{Q}$ forms a reflexive graph with identities given by the case where $M=i d^{\bullet}$, which forms a graph with complementarity as follows.

Let $(\mathbb{I} \mathbb{Q})^{\mathbb{W}}$ consist of all closed-world quasi-moves in $\mathbb{I} \mathbb{Q}$. For any $h: I \rightarrow X, k: J \rightarrow Y$, and $c: K \rightarrow Z$, let $(h, k) \triangleright^{\mathbb{I Q}} c$ iff $I=J=K, Z=h+_{I} k$, and $c$ is the corresponding map $I \rightarrow Z$. On edges, for any $M_{h}: h^{\prime} \rightarrow h, M_{k}: k^{\prime} \rightarrow k$, and $M_{c}: c^{\prime} \rightarrow c$, let $\left(M_{h}, M_{k}\right) \triangleright^{\mathbb{I Q}} M_{c}$ iff there exists a diagram

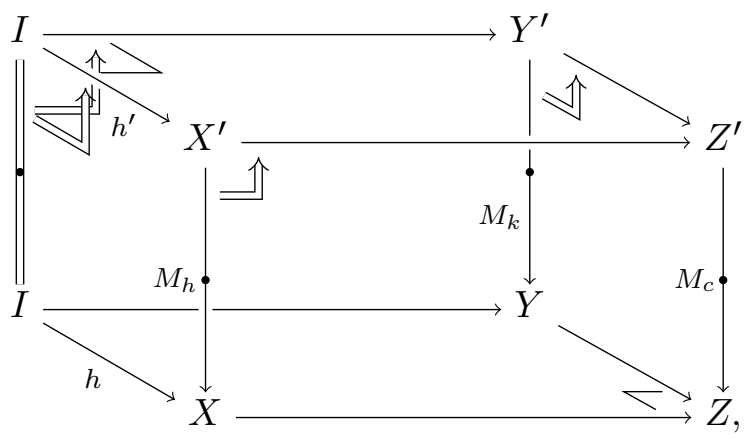

where $M_{c}$ is a closed-world quasi-move and double cells with a 'double pullback' mark are cartesian, as below Axiom (P1) (page 25). (One easily shows that the upper square is also a pushout.) Then $(\mathbb{I} \mathbb{Q})^{\circ}$, consists of all pairs $\left(M_{h}, M_{k}\right)$ for which there exists a diagram of the shape (6.2).

Let $\ell^{\mathbb{Q} Q}$ be the composite $(\mathbb{I Q})^{\mathbb{W}} \hookrightarrow \mathbb{W}_{C C S} \stackrel{\ell_{\mathbb{D}}}{\longrightarrow} \Sigma$. It thus maps tick moves to $\odot$ and all other closed-world moves to $i d$. The composite $(\mathbb{I} \mathbb{Q})^{2} \stackrel{\triangleright^{\mathbb{Q}}}{\rightarrow}(\mathbb{I} \mathbb{Q})^{\mathbb{W}} \rightarrow \Sigma$ is indeed partially functional and symmetric.

There is an obvious morphism $\chi: \mathbb{I} \rightarrow \mathbb{Q}$ of reflexive graphs. 
Example 6.6. Recall the alphabet $\mathbb{A}$ for CCS. It also forms a graph with complementarity, as follows. Let $\mathbb{A}^{\mathbb{W}}$ consist of all vertices and of all $\nabla$ and $i d$ edges. Let $\mathbb{A}^{\subsetneq}$ consist, on vertices, of the diagonal, i.e., all pairs $(n, n)$. On edges, let $e \leftrightharpoons e^{\prime}$ when $\operatorname{dom}(e) \subsetneq \operatorname{dom}\left(e^{\prime}\right)$ and:

- one of $e$ and $e^{\prime}$ is in $\mathbb{A}^{\mathbb{W}}$, the other being an identity,

- or one of $e$ and $e^{\prime}$ is an input on some $i \in \operatorname{dom}(e)$, the other being an output on $i$.

Define now our relation $\triangleright^{\mathbb{A}}$ to be the graph of the map sending all coherent pairs $e \smile e^{\prime}$ to $i d$, except when one is a $\varnothing$, in which case the pair is sent to $\nabla: n \rightarrow n$. The axioms are easily satisfied.

Let $\xi: \mathbb{I Q} \rightarrow \mathbb{A}$ map any vertex $h: I \rightarrow X$ to $n=I(\star)$, and any edge (6.1) to

- $i d_{n}$ if $M$ is an identity, a synchronisation, a fork, or a channel creation,

- $\nabla_{n}$ if $M$ is a tick move,

- $i$ if $M$ is an input on $h_{\star}(i)$,

- $\bar{i}$ if $M$ is an output on $h_{\star}(i)$.

This map $\xi$ is a morphism of graphs with complementarity.

We have the following general way of constructing graphs with complementarity. For any graph with complementarity $A$ and morphism of reflexive graphs $p: G \rightarrow A$, consider the following candidate complementarity structure on $G$.

Let $G^{\mathbb{W}}=G \times_{A} A^{\mathbb{W}}$ denote the pullback

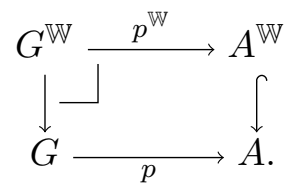

Further, let $\ell^{G}$ be the composite $G^{\mathbb{W}} \stackrel{p^{\mathbb{W}}}{\longrightarrow} A^{\mathbb{W}} \stackrel{\ell^{A}}{\longrightarrow} \Sigma$, and let $(x, y) \triangleright^{G} z$ iff $(p(x), p(y)) \triangleright^{A} p(z)$ (for both vertices and edges). In other words, $\triangleright^{G}$ is the relational composite

$$
G^{2} \stackrel{p^{2}}{\rightarrow} A^{2} \stackrel{\triangleright^{A}}{\longrightarrow} A^{\mathbb{W}} \stackrel{p^{\mathbb{W}}}{\longleftarrow} G^{\mathbb{W}}
$$

where the backwards $p^{\mathbb{W}}$ arrow denotes the converse of the graph of $p^{\mathbb{W}}$.

Proposition 6.7. For any subrelation $R \subseteq \triangleright^{G}$, if $R$ is symmetric, then $\left(G, G^{\mathbb{W}}, R, \ell^{G}\right)$ forms a graph with complementarity, and $p$ is a morphism of graphs with complementarity to $A$.

Proof. By standard relational algebra, the composite relation

$$
G^{2} \stackrel{\triangleright^{G}}{\longrightarrow} G^{\mathbb{W}} \stackrel{p^{\mathbb{W}}}{\longrightarrow} A^{\mathbb{W}},
$$

which is equal to

$$
G^{2} \stackrel{p^{2}}{\longrightarrow} A^{2} \stackrel{\triangleright^{A}}{\longrightarrow} A^{\mathbb{W}} \stackrel{p^{\mathbb{W}}}{\longleftarrow} G^{\mathbb{W}} \stackrel{p^{\mathbb{W}}}{\longrightarrow} A^{\mathbb{W}}
$$

is included in

$$
G^{2} \stackrel{p^{2}}{\rightarrow} A^{2} \stackrel{\triangleright^{A}}{\longrightarrow} A^{\mathbb{W}}
$$

Composing with $\ell^{A}$, we obtain that $\left(\ell^{G} \circ \triangleright^{G}\right) \subseteq\left(\ell^{A} \circ \triangleright^{A} \circ p^{2}\right)$, which is straightforwardly symmetric and partially functional. A subrelation of a partially functional relation is automatically partially functional, so $\ell^{G} \circ R$ is partially functional. It is symmetric because $R$ is, hence the result. 
Example 6.8. $C C S$ forms a graph with complementarity over $\mathbb{A}$ by the last proposition, taking $R$ to relate

- all pairs $(n \vdash P, n \vdash Q)$ to $n \vdash P \mid Q$ on vertices,

- any transitions $\left(\Gamma \vdash P_{1}\right) \stackrel{\alpha}{\leftarrow}\left(\Gamma \vdash P_{1}^{\prime}\right)$ and $\left(\Gamma \vdash P_{2}\right) \stackrel{i d}{\longleftarrow}\left(\Gamma \vdash P_{2}\right)$ with $\left(\Gamma \vdash P_{1} \mid P_{2}\right) \stackrel{\alpha}{\leftarrow}(\Gamma \vdash$ $\left.P_{1}^{\prime} \mid P_{2}\right)$, and symmetrically,

- and any two transitions $\left(\Gamma \vdash P_{1}\right) \stackrel{\alpha}{\leftarrow}\left(\Gamma \vdash P_{1}^{\prime}\right)$ and $\left(\Gamma \vdash P_{2}\right) \stackrel{\bar{\alpha}}{\leftarrow}\left(\Gamma \vdash P_{2}^{\prime}\right)$ with $(\Gamma \vdash$ $\left.P_{1} \mid P_{2}\right) \stackrel{i d}{\longleftarrow}\left(\Gamma \vdash P_{1}^{\prime} \mid P_{2}^{\prime}\right)$.

Proposition 6.9. Suppose given a choice, for all $x, y \in G$ and $a \in A$ such that $(p(x), p(y)) \triangleright^{A}$ $a$, of a vertex $[x, y]_{a} \in G$ such that $p\left([x, y]_{a}\right)=a$, satisfying the following condition: for all edges $e_{x}: x^{\prime} \rightarrow x$ and $e_{y}: y^{\prime} \rightarrow y$ in $G$, and $e_{a}: a^{\prime} \rightarrow a$ in $A$, if $\left(p\left(e_{x}\right), p\left(e_{y}\right)\right) \triangleright^{A} e_{a}$, then there exists $a\left[e_{x}, e_{y}\right]_{e_{a}}:\left[x^{\prime}, y^{\prime}\right]_{a^{\prime}} \rightarrow[x, y]_{a}$ such that $p\left(\left[e_{x}, e_{y}\right]_{e_{a}}\right)=e_{a}$.

Then, $\left(G, G^{\mathbb{W}}, \triangleright^{G}, \ell^{G}\right)$ forms a graph with complementarity, and $p$ is a morphism of graphs with complementarity.

Proof. Recalling the beginning of the proof of Proposition 6.7, the hypothesis implies that the inclusion

$$
\left(G^{2} \stackrel{\triangleright^{G}}{\rightarrow} G^{\mathbb{W}} \stackrel{p^{\mathbb{W}}}{\longrightarrow} A^{\mathbb{W}}\right) \subseteq\left(G^{2} \stackrel{p^{2}}{\rightarrow} A^{2} \stackrel{\triangleright^{A}}{\rightarrow} A^{\mathbb{W}}\right)
$$

is actually an equality.

Composing with $\ell^{A}$, we obtain that $\ell^{G} \circ \triangleright^{G}=\ell^{A} \circ \triangleright^{A} \circ p^{2}$, which is straightforwardly symmetric and partially functional. The morphism $p$ is a morphism of graphs with complementarity by construction.

Definition 6.10. Let $\mathcal{S}^{\mathbb{Q}}=\Delta_{\chi}(\mathcal{S})$ and $\mathcal{T}^{\mathbb{Q}}=\Delta_{\chi}(\mathcal{T})$ be the pullbacks of $\mathcal{S} \rightarrow \mathbb{Q}$ and $\mathcal{T} \rightarrow \mathbb{Q}$ along $\chi: \mathbb{I} \mathbb{Q} \rightarrow \mathbb{Q}$.

Example 6.11. $\mathcal{S}^{\mathbb{I} Q}$ and $\mathcal{T}^{\mathbb{I} Q}$ form graphs with complementarity over $\mathbb{I} \mathbb{Q}$ by Proposition 6.9. The canonical relation $\triangleright^{C C S}$ does not satisfy the condition of Proposition [6.9, however. Indeed, e.g., any non-silent transition $\left(\Gamma \vdash P_{1}\right) \stackrel{\alpha}{\leftarrow}\left(\Gamma \vdash P_{1}^{\prime}\right)$ and silent but non-identity transition $\left(\Gamma \vdash P_{2}\right) \stackrel{i d}{\longleftarrow}\left(\Gamma \vdash P_{2}^{\prime}\right)$ are not coherent in $C C S$, although their images under the projection to $\mathbb{A}$ are so. (Amalgamating two such transitions in $C C S$ requires a path of length 2, as will be used below.) What saves $\mathcal{S}^{\mathbb{I Q}}$ and $\mathcal{T}^{\mathbb{Q}}$ from this issue is that projecting to $\mathbb{I} \mathbb{Q}$ does not hide away, e.g., synchronisations.

6.2. Modular graphs and fair testing equivalence. We now introduce the notion of modular graph, which is appropriate for defining fair testing. We could actually introduce fair testing for arbitrary graphs with complementarity, but the extra generality would make little sense.

For any graph with complementarity $G, G^{\odot}$ forms an LTS over $\Sigma$, through $G^{\odot} \stackrel{\Downarrow}{\rightarrow} \Sigma$.

Definition 6.12. $G$ is modular iff for all $(x, y) \triangleright^{G} z$ we have both:

(1) for all $e: z^{\prime} \rightarrow z$, there exists $e_{x}: x^{\prime} \rightarrow x$ and $e_{y}: y^{\prime} \rightarrow y$ such that $\left(e_{x}, e_{y}\right) \triangleright^{G} e$; and

(2) for all $e_{x}: x^{\prime} \rightarrow x$ and $e_{y}: y^{\prime} \rightarrow y$ such that $e_{x}=e_{y}$ there exists $e: z^{\prime} \rightarrow z$ such that $\left(e_{x}, e_{y}\right) \triangleright^{G} e$. 
Remark 6.13. The second condition is almost redundant: in any graph with complementarity $G$, there exists $e^{\prime}$ such that $\left(e_{x}, e_{y}\right) \triangleright^{G} e^{\prime}$, but the target of $e^{\prime}$ may be any $u$ such that $(x, y) \triangleright^{G} u$; it does not have to be $z$.

Proposition 6.14. $G$ is modular iff $\triangleright^{G}$ is a strong bisimulation over $\Sigma$.

We here implicitly view $\triangleright^{G}$ as a relation $G^{\odot} \longrightarrow G^{\mathbb{W}}$.

Proof. Since $\triangleright^{G}$ is a relation over $\Sigma$, it is enough to prove that both projections are graph fibrations, which is directly equivalent to modularity.

Example 6.15. $\mathcal{S}^{\mathbb{I} Q}$ and $\mathcal{T}^{\mathbb{Q} Q}$, as well as $C C S$, are modular.

We now define fair testing in any modular graph, and compare with both semantic fair testing equivalence $\left(\sim_{f}\right)$ for strategies and standard fair testing equivalence $\left(\sim_{f, s}\right)$ for CCS processes. Recall that $\sim_{\Sigma}$ denotes strong bisimilarity over $\Sigma$.

Lemma 6.16. For any modular graph with complementarity $G$ and $x, y, z, t \in G$, if $(x, y) \triangleright^{G}$ $z$ and $(x, y) \triangleright^{G} t$, then $z \sim_{\Sigma} t$.

Proof. We have $z \sim_{\Sigma}(x, y) \sim_{\Sigma} t$.

Any modular graph may be equipped with a choice of $z$ such that $(x, y) \triangleright^{G} z$, for all $x \frown y$. We denote such a choice by $[x, y]$. By the lemma, the choice of $z$ does not matter as long as we only consider properties invariant under $\sim_{\Sigma}$. Here, we only need the standard predicate for fair testing.

Definition 6.17. For any reflexive graph $G$ over $\Sigma$, let $\perp^{G}$ denote the set of all $x \in G$ such that for all $x \Leftarrow x^{\prime}$ there exists $x^{\prime} \stackrel{\ominus}{=} x^{\prime \prime}$.

When $G$ is a graph with complementarity, we often denote $\perp^{G^{\mathbb{W}}}$ by $\perp^{G}$. There is no confusion because $G$ is not even a graph over $\Sigma$ in general.

In any modular graph with complementarity $G$, let, for any $x \in G, x^{\curvearrowright}=\{y \mid x \frown y\}$, and let $x \bowtie y$ iff $x^{\odot}=y^{\curvearrowright}$.

Definition 6.18. For any $x, y \in G$, let $x \sim_{f}^{G} y$ iff $x \bowtie y$ and for all $z \in x^{\curvearrowright},[x, z] \in \perp^{G}$ iff $[y, z] \in \perp^{G}$.

We may at last define fair relations:

Definition 6.19. For all modular graphs with complementarity $G$ and $H$, and full relations $R: G \rightarrow H$, let $R$ preserve fair testing equivalence when, for all $x R x^{\prime}$ and $y R y^{\prime},\left(x \sim_{f}^{G} y\right)$ implies $\left(x^{\prime} \sim_{f}^{H} y^{\prime}\right)$. $R$ reflects fair testing equivalence when the converse implication holds. $R$ is fair when it preserves and reflects fair testing equivalence.

Modularity enables a first, easy characterisation of fair testing.

Proposition 6.20. If $G$ is modular, then for any $x \frown y,[x, y] \in \perp^{G}$ iff $(x, y) \in \perp^{G^{\circ}}$.

Proof. A direct consequence of Proposition 6.14.

We now prove that the general definition of fair testing equivalence instantiates correctly for $\mathcal{S}^{\mathbb{I Q}}$ and $C C S$. First, we easily have

Proposition 6.21. For any two CCS processes $P$ and $Q$ over $n, P \sim_{f, s} Q$ iff $P \sim_{f}^{C C S} Q$.

Proof. Straightforward. 
We now wish to compare $\perp^{\mathcal{S}^{\mathbb{Q}}}$, as defined in this section, and the semantic $\Perp$. As an intermediate step, we consider the following, bare $\mathcal{L}$, which lives over $\mathbb{Q}$, but is defined in terms of LTSs (as opposed to successful states of strategies). Let $\mathcal{S}^{\mathbb{W}}$ be the restriction of $\mathcal{S}$ to closed-world transitions, i.e., the pullback of $\mathcal{S} \rightarrow \mathbb{Q}$ along the inclusion $\mathbb{W}_{C C S} \hookrightarrow \mathbb{Q}$; this is an LTS over $\Sigma$ via $\ell_{\mathbb{D}}$. Let $\mathcal{L}=\perp^{\mathcal{S}^{\mathbb{W}}}$ denote the set of pairs $(X, S) \in \mathcal{S}$ such that for all $(X, S) \Leftarrow\left(X^{\prime}, S^{\prime}\right)$ there exists $\left(X^{\prime}, S^{\prime}\right) \stackrel{\ominus}{\Longleftrightarrow}\left(X^{\prime \prime}, S^{\prime \prime}\right)$.

Lemma 6.22. For all $(X, S) \in \mathcal{S}, \bar{S} \in \Perp_{X}$ iff $(X, S) \in \mathcal{L}$.

This essentially amounts to checking that the notions of closed-world, successful, and unsuccessful play (Definition 3.22), correspond with closed-world, successful, and unsuccessful transition sequences. The former are defined in terms of plays and moves therein, while the latter rest upon the map $\ell_{\mathbb{D}}: \mathbb{W}_{C C S} \rightarrow \Sigma$.

We first observe:

Lemma 6.23. For any two closed-world plays $W, W^{\prime}$ over $X$, and $\alpha: W \rightarrow W^{\prime}$ in $Ð(X), \alpha$ is an isomorphism, and it is unique.

Proof of Lemma 6.22. Let $S \in S S_{X}$ and assume $\bar{S} \in \Perp_{X}$. Let $S \Leftarrow S^{\prime}$ (over $\Sigma$ ). This means that there exists a path $p$

$$
X=X_{0} \stackrel{M_{1}}{\longleftarrow} X_{1} \stackrel{M_{2}}{\longleftarrow} \ldots X_{n}=X^{\prime},
$$

such that, omitting positions,

$$
S=S_{0} \stackrel{M_{1}}{\longleftarrow} S_{1} \stackrel{M_{2}}{\longleftarrow} \ldots S_{n}=S^{\prime}
$$

and $p$ is mapped by $\ell_{\mathbb{D}}^{\star}$ to the path of length $n$ consisting only of $i d$ edges. This implies by induction the existence of $\sigma \in \bar{S}(W)$, where $W=M_{1} \bullet \ldots \bullet M_{n}$ is closed-world and unsuccessful, such that $S^{\prime}=(S \cdot W)_{\mid \psi(\sigma)}$. Because $\bar{S} \in \Perp_{X}$, there exists a successful, closedworld play $W^{\prime}$, a morphism $f: W \rightarrow W^{\prime}$ in $\mathbb{E}(X)$, and $\sigma^{\prime} \in \bar{S}\left(W^{\prime}\right)$ such that $\sigma^{\prime} \cdot f=\sigma$. By Lemma 6.23. $W^{\prime}$ is isomorphic to an extension of $W$ with closed-world moves, say $W^{\prime} \cong W \bullet M_{n+1} \bullet \ldots \bullet M_{n+m}$. By induction on $m$, we obtain a path

$$
S^{\prime}=S_{n} \stackrel{M_{n+1}}{\longleftarrow} S_{n+1} \stackrel{M_{n+2}}{\longleftarrow} \ldots S_{n+m}
$$

where $S_{n+m}=\left(S \cdot W^{\prime}\right)_{\mid \psi\left(\sigma^{\prime}\right)}$. Because $W^{\prime}$ is successful, there exists $i \in m$ such that $\ell_{\mathbb{D}}\left(M_{n+i}\right)=$

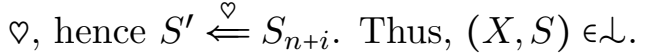

Conversely, assume $(X, S) \in \mathcal{L}$. Let $W$ be an unsuccessful, closed-world play over $X$ and $\sigma \in \bar{S}(W)$. Picking a decomposition $W=M_{1} \bullet \ldots \bullet M_{n}$ of $W$, we obtain a path $p$

$$
S=S_{0} \stackrel{M_{1}}{\longleftarrow} S_{1} \ldots \stackrel{M_{n}}{\longleftarrow} S_{n}=S^{\prime}
$$

in $\mathcal{S}$ such that $S^{\prime}=(S \cdot W)_{\mid \psi(\sigma)}$, which yields $S \Leftarrow S^{\prime}$. Because $(X, S) \in \mathcal{L}$, there exists $S^{\prime} \Leftarrow S^{\prime \prime} \stackrel{\odot}{\leftarrow} S^{\prime \prime \prime}$, with underlying path

$$
S^{\prime}=S_{n} \stackrel{M_{n+1}}{\longleftarrow} S_{n+1} \ldots \stackrel{M_{n+m}}{\longleftarrow} S_{n+m}=S^{\prime \prime} \stackrel{M_{n+m+1}}{\longleftarrow} S^{\prime \prime \prime}
$$

in $\mathcal{S}^{\mathbb{W}}$, such that $\ell_{\mathbb{D}}\left(M_{n+i}\right)=i d$ for all $i \in m$ and $\ell_{\mathbb{D}}\left(M_{n+m+1}\right)=\varnothing$. But by definition this means that $S^{\prime \prime \prime}=\left(S^{\prime} \cdot W^{\prime}\right)_{\mid \psi\left(\sigma^{\prime}\right)}$ for some

$$
\sigma^{\prime} \in \overline{S^{\prime}}\left(W^{\prime}\right)=\overline{(S \cdot W)_{\mid \psi(\sigma)}}\left(W^{\prime}\right)=\left\{\sigma^{\prime \prime} \in \bar{S}\left(W \bullet W^{\prime}\right) \mid \sigma^{\prime \prime} \cdot f=\sigma\right\},
$$


where $W^{\prime}=M_{n+1} \bullet \ldots \bullet M_{n+m+1}$ and $f: W \rightarrow\left(W \bullet W^{\prime}\right)$ is the extension. By construction, $\sigma^{\prime} \cdot f=\sigma$. Hence, $\bar{S} \in \Perp_{X}$.

We furthermore have:

Lemma 6.24. For any vertex $h: I \rightarrow X$ of $\mathbb{I} \mathbb{Q}$ and $S \in S S_{X},(I, h, S) \in \perp^{\mathcal{S}^{\mathbb{I Q}}}$ iff $(X, S) \in \mathcal{L}$.

Proof. The map $\chi^{\mathbb{W}}: \mathbb{Q}^{\mathbb{W}} \rightarrow \mathbb{Q}^{\mathbb{W}}$ is a strong, functional bisimulation, because for any $h: I \rightarrow$ $X$ and closed-world move $M: Y \rightarrow X$, there exists a diagram (6.1). Thus, the projection $\left(\mathcal{S}^{\mathbb{I Q}}\right)^{\mathbb{W}} \rightarrow \mathcal{S}^{\mathbb{W}}$ is a strong, functional bisimulation by Proposition 2.11.

Remark 6.25. Interfaces are pretty irrelevant here, and indeed we could have decreed that closed-world moves only relate vertices with empty interfaces in $\mathbb{I Q}$. This is unnecessary here, though, so we stick to the simpler definition, but it will be crucial for the $\pi$-calculus.

This entails:

Corollary 6.26. For any $h: I \rightarrow X, h^{\prime}: I \rightarrow X^{\prime}, S \in S S_{X}$, and $S^{\prime} \in S S_{X^{\prime}},(I, h, S) \sim_{f}$ $\left(I, h^{\prime}, S^{\prime}\right)$ iff $(I, h, S) \sim_{f}^{\mathbb{I} Q \mathbb{Q}}\left(I, h^{\prime}, S^{\prime}\right)$.

Proof. We have

$$
\begin{aligned}
& (I, h, S) \sim_{f}\left(I, h^{\prime}, S^{\prime}\right) \\
& \Uparrow(\text { by definition) } \\
& \forall Y, k: I \rightarrow Y, T \in S S_{Y},\left(\overline{[S, T]} \in \Perp_{X+{ }_{I} Y} \Leftrightarrow \overline{\left[S^{\prime}, T\right]} \in \Perp_{X^{\prime}+{ }_{I} Y}\right) \\
& \Uparrow \text { (by Lemma 6.22) } \\
& \forall Y, k: I \rightarrow Y, T \in S S_{Y},\left(\left(X+{ }_{I} Y,[S, T]\right) \in \mathcal{L} \Leftrightarrow\left(X^{\prime}+_{I} Y,\left[S^{\prime}, T\right]\right) \in \mathcal{L}\right) \\
& \Uparrow \text { (by Lemma 6.24) } \\
& \forall Y, k: I \rightarrow Y, T \in S S_{Y},\left(\left(I \rightarrow X+_{I} Y,[S, T]\right) \in \perp^{\mathcal{S}^{\mathbb{I Q}}} \Leftrightarrow\left(I \rightarrow X^{\prime}+_{I} Y,\left[S^{\prime}, T\right]\right) \in \perp^{\mathcal{S}^{\mathbb{I Q}}}\right) \\
& \Uparrow \text { (by definition) } \\
& (I, h, S) \underset{f}{\mathcal{S}_{f}^{\mathbb{Q}}}\left(I, h^{\prime}, S^{\prime}\right) \text {, }
\end{aligned}
$$

which concludes the proof.

6.3. Adequacy. Until now, our study of graphs with complementarity and fair testing therein is intrinsic, i.e., fair testing equivalence in a modular graph with complementarity $G$ does not depend on any alphabet. We now address the question of what an alphabet should be, for $G$. The main idea is that such an alphabet $A$ should be a graph with complementarity, and that viewing it as an alphabet for $G$ is the same as providing a morphism $p: G \rightarrow A$ in GCompl, satisfying a certain condition called adequacy. To understand the role of this condition, one should realise that edges in $G$ may be much too fine a tool for checking fair testing equivalence. E.g., in $\mathcal{S}^{\mathbb{I} Q}$, they include information about which players played which move. Thus, although it is true that weak bisimilarity implies fair testing equivalence, this property is essentially useless for fair testing, because too few strategies are weakly bisimilar. Any morphism $p: \mathcal{S}^{\mathbb{Q}} \rightarrow A$ induces an a priori coarser version of fair testing for $\mathcal{S}^{\mathbb{I Q}}$, where one only looks at labels in $A$. Adequacy is a sufficient condition for this latter version to coincide with the original. This will in particular entail that weak bisimilarity over $A$ is finer than fair testing equivalence.

Adequacy relies on the following:

Definition 6.27. Consider, for any $p: G \rightarrow A$ and $q: H \rightarrow A$ the pullback 


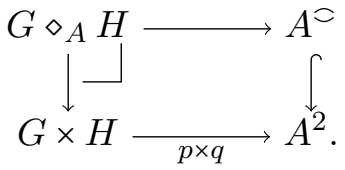

We call $G \diamond_{A} H$ the blind composition of $G$ and $H$ over $A$, viewed as an LTS over $\Sigma$ via $G \diamond_{A} H \rightarrow A^{\ulcorner} \rightarrow \Sigma$.

Recall from Section 2.2 that $\approx_{A}$ denotes weak bisimilarity for reflexive graphs over $A$.

Definition 6.28. Let $p: G \rightarrow A$ be a morphism of graphs with complementarity. We say that $p$ is adequate iff

- the graph of ob $G^{\circ} \hookrightarrow \operatorname{ob}\left(G \diamond_{A} G\right)$ is included in $\approx_{\Sigma}$, and

- for all $x, y \in \mathrm{ob}(G), x \frown y$ iff $p(x) \frown p(y)$.

Concretely, any transition $\left(e_{1}, e_{2}\right) \in G^{\odot}$ is matched, without any hypothesis on $G$, by $\left(e_{1}, e_{2}\right)$ itself. Conversely, having a transition $\left(x_{1}, x_{2}\right) \stackrel{e_{1}, e_{2}}{\longleftarrow}\left(x_{1}^{\prime}, x_{2}^{\prime}\right)$ in $G \diamond_{A} G$ means that $p\left(e_{1}\right) \Downarrow p\left(e_{2}\right)=\sigma$. Adequacy demands that there exists a path $\left(r_{1}, r_{2}\right):\left(x_{1}, x_{2}\right) \leftarrow^{\star}\left(x_{1}^{\prime \prime}, x_{2}^{\prime \prime}\right)$ in $G^{\circ}$, such that $\widetilde{r_{1} \Downarrow^{\star} r_{2}}=\widetilde{(\sigma)}$, and $\left(x_{1}^{\prime \prime}, x_{2}^{\prime \prime}\right) \approx_{\Sigma}\left(x_{1}^{\prime}, x_{2}^{\prime}\right)$, where the left-hand side is in $G^{\circ}$ and the right-hand side is in $G \diamond_{A} G$.

Recall the map $\xi: \mathbb{I} \mathbb{Q} \rightarrow \mathbb{A}$ from Example 6.6. Via this map, $\mathcal{S}^{\mathbb{Q} Q}$ and $\mathcal{T}^{\mathbb{I} Q}$ form LTSs and even graphs with complementarity over $\mathbb{A}$.

Proposition 6.29. The maps from $C C S, \mathcal{S}^{\mathbb{I}}$, and $\mathcal{T}^{\mathbb{I} Q}$ to $\mathbb{A}$ are adequate.

Proof. For all three graphs $p: G \rightarrow \mathbb{A}$ over $\mathbb{A}$, both $G^{\circ}$ and $G \diamond_{A} G$ form graphs over $\mathbb{A}^{\mathbb{W}}$, because $\triangleright^{\mathbb{A}}: \mathbb{A}^{2} \longrightarrow \mathbb{A}^{\mathbb{W}}$ is actually partially functional. In each case, the graph of ob $G^{\odot} \hookrightarrow$ $\mathrm{ob}\left(G \diamond_{A} G\right)$ is a weak bisimulation over $\mathbb{A}^{\mathbb{W}}$, because for all $e$ and $e^{\prime}$ in $G$, if $p(e) \frown p\left(e^{\prime}\right)$, then either $e \leftrightharpoons e^{\prime}$, or both interleavings are coherent, i.e., $(e, i d) \leftrightharpoons\left(i d, e^{\prime}\right)$ and $(i d, e) \frown\left(e^{\prime}, i d\right)$, pointwise. (Here, e.g., $(e, i d)$ denotes the path $\cdot \stackrel{e}{\leftarrow} \cdot \stackrel{i d}{\longleftarrow} \cdot$.)

The only subtle point is that this only holds thanks to the restrictions put on edges of $\mathbb{I} \mathbb{Q}$. E.g., consider the graph the graph $\mathbb{I} \mathbb{Q}_{-}$with the same vertices as $\mathbb{I} \mathbb{Q}$, and edges $(I \stackrel{k}{\rightarrow} Y) \rightarrow(I \stackrel{h}{\rightarrow} X)$ given just as for $\mathbb{I} \mathbb{Q}$, except that we do not require existence of a diagram (6.1). Pullback yields a graph $\mathcal{S}_{-}$over $\mathbb{I} \mathbb{Q}_{-}$. Extending $\xi$ to $\xi^{\prime}: \mathbb{I} \mathbb{Q}_{-} \rightarrow \mathbb{A}$ in the obvious way, we obtain a graph over $\mathbb{A}$. Consider now the moves $o_{2,1}, \iota_{2,1}:[2] \rightarrow[2]$, let $I=2 \cdot \star$, and let $f$ be one of the two embeddings $I \rightarrow[2]$, say the one which is an inclusion at $\star, f^{\prime}$ being the other. Recalling labels in $\mathbb{A}$ from Definition 2.20, we have edges $(I, f,[2]) \stackrel{\overline{1}}{\leftarrow}(I, f,[2])$ and $(I, f,[2]) \stackrel{1}{\leftarrow}\left(I, f^{\prime},[2]\right)$, and $(I, f,[2]) \frown(I, f,[2])$. However, the two edges are not coherent, because any attempt to construct a diagram (6.2) (with here $h=h^{\prime}=k=f$, and $k^{\prime}=f^{\prime}$ ) fails (even if we forget about the vertical identity). This is the very reason we use $\mathbb{I} \mathbb{Q}$ instead of $\mathbb{I} \mathbb{Q}$.

We have the following two easy properties of blind composition.

Proposition 6.30. For any modular $G$, adequate $p: G \rightarrow A$, and $x \subset y$ in $G$, we have $[x, y] \in \perp^{G}$ iff $(x, y) \in \perp^{G \diamond_{A} G}$.

Proof. We have $[x, y] \approx_{\Sigma}\left((x, y) \in G^{\complement}\right) \approx_{\Sigma}\left((x, y) \in G \diamond_{A} G\right)$. 
Proposition 6.31. For any $H$ over $A$, modular $G$, adequate $p: G \rightarrow A, x_{1}, x_{2} \in G$, and $y$ in $H$, if $x_{2} \approx_{A} y$, then

$$
\left[x_{1}, x_{2}\right] \in \perp^{G} \text { iff }\left(x_{1}, y\right) \in \perp^{G \diamond_{A} H} .
$$

Proof. By Proposition 6.30, it is enough to prove that the right-hand side is equivalent to $\left(x_{1}, x_{2}\right) \in \perp^{G \diamond_{A} G}$, which is straightforward by hypothesis.

We conclude this section by stating the main property of blind composition, Proposition 6.37 below, which will be used extensively in the next section.

To start with, recall the following notation from Section 2.2.1. There, considering a morphism $p: G \rightarrow A$ of reflexive graphs, we defined $x \underset{A}{\stackrel{r}{\rightleftarrows}} x^{\prime}$, for $x, x^{\prime} \in \mathrm{ob}(G)$ and $r: p(x) \leftarrow p\left(x^{\prime}\right)$ in $A^{\star}$. Namely, this denotes any path $r^{\prime}: x \leftarrow^{\star} x^{\prime}$ in $G$, such that $\overline{p^{\star}\left(r^{\prime}\right)}=\widetilde{r}$.

In order to state Proposition 6.37, we now need to equip $\mathrm{fc}(A)$ with complementarity structure, but we cannot do it over the graph $\Sigma$, because closed-world paths may contain more than one $\odot$ edge, hence cannot all be mapped to $\Sigma$. We thus define categories with complementarity.

The notions of relation, partial functionality, functionality, totality, and domain on reflexive graphs carry over to categories, e.g., a relation $A \longrightarrow B$ is a subcategory $R \subseteq A \times B$. The only subtlety is that the definitions imply certain functoriality properties. E.g., for any composites $g \circ f$ in $A$ and $g^{\prime} \circ f^{\prime}$ in $B$, if $\left(f, f^{\prime}\right) \in R$ and $\left(g, g^{\prime}\right) \in R$, because $R$, as a subcategory, is stable under composition, we have for free that $\left(g \circ f, g^{\prime} \circ f^{\prime}\right) \in R$. Similarly, if $(x, y) \in R$ for objects $x \in A$ and $y \in B$, then $\left(i d_{x}, i d_{y}\right) \in R$. We thus rename partial functionality and functionality into partial functoriality and functoriality in this setting.

Definition 6.32. A category with complementarity is a category $A$, equipped with a subcategory $A^{\mathbb{W}}$, a relation $\triangleright^{A}: A^{2} \longrightarrow A^{\mathbb{W}}$, and a functor $\ell^{A}: A^{\mathbb{W}} \rightarrow \mathrm{fc}(\Sigma)$, such that the composite $A^{2} \mapsto A^{\mathbb{W}} \rightarrow \mathrm{fc}(\Sigma)$ is partially functorial and symmetric.

Again, we let $A^{\circ}=\operatorname{dom}\left(\triangleright^{A}\right)$ and write $a \bigodot a^{\prime}$ for $\left(a, a^{\prime}\right) \in A^{\circ}$. We further denote the map $A^{\complement} \hookrightarrow A^{2} \longrightarrow A^{\mathbb{W}} \rightarrow \mathrm{fc}(\Sigma)$ by $(a, b) \mapsto(a \Downarrow b)$, and deem morphisms in $A^{\mathbb{W}}$ closed-world.

Defining functors with complementarity in the obvious way, we obtain:

Proposition 6.33. Categories with complementarity form a (locally small) category CCompl.

Consider the functor UCompl:CCompl $\rightarrow$ GCompl mapping any category with complementarity $\mathbb{C}$ to its underlying graph, say $G$, which we equip with complementarity structure as follows. First, define $G^{\mathbb{W}}$ and $\ell^{G}$ by the pullback

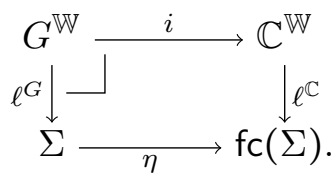

Furthermore, let $\triangleright^{\mathbb{C}}$ consist of all triples $(x, y, z)$ of vertices (resp. edges) such that $z \in G^{\mathbb{W}}$ and $(x, y) \triangleright^{\mathbb{C}} z$ (which is a pullback of $\left(\triangleright^{\mathbb{C}}\right) \hookrightarrow \mathbb{C}^{2} \times \mathbb{C}^{\mathbb{W}}$ along $\mathbb{C}^{2} \times G^{\mathbb{W}} \hookrightarrow \mathbb{C}^{2} \times \mathbb{C}^{\mathbb{W}}$ ). This clearly equips $G$ with complementarity structure and extends to the announced functor UCompl.

This functor does not appear to have a left adjoint, because complementarity in $G$ may behave badly w.r.t. composition in $\mathrm{fc}(G)$. However, we may define the following candidate structure on $\mathrm{fc}(G)$. Consider any graph with complementarity $G$, and let us 
start by defining a complementarity structure on $G^{\star}$. Let $\left(G^{\star}\right)^{\mathbb{W}}$ denote the subcategory of closed-world paths in $G$, i.e., $\left(G^{\star}\right)^{\mathbb{W}}=\left(G^{\mathbb{W}}\right)^{\star}$. Accordingly, let $\ell^{G^{\star}}$ be the composite

$$
\left(G^{\mathbb{W}}\right)^{\star} \stackrel{\left(\ell^{G}\right)^{\star}}{\longrightarrow} \Sigma^{\star} \stackrel{\sim}{\rightarrow} \mathrm{fc}(\Sigma) .
$$

Finally, consider the functor

$$
\left(G^{2}\right)^{\star} \stackrel{\left\langle\pi^{\star},\left(\pi^{\prime}\right)^{\star}\right\rangle}{\longrightarrow}\left(G^{\star}\right)^{2} .
$$

It yields a relation $\left(G^{2}\right)^{\star} \longrightarrow\left(G^{\star}\right)^{2}$, whose converse we use to define $\triangleright^{G^{\star}}$ as the composite relation

$$
\left(G^{\star}\right)^{2} \stackrel{\left\langle\pi^{\star},\left(\pi^{\prime}\right)^{\star}\right\rangle^{\dagger}}{\longrightarrow}\left(G^{2}\right)^{\star} \stackrel{\left(\triangleright^{G}\right)^{\star}}{\longrightarrow} G^{\star} .
$$

Concretely, $\triangleright^{G^{\star}}$ is $\triangleright^{G}$ on objects, and on paths, we have $\left(r_{1}, r_{2}\right) \triangleright^{G^{\star}} r$ iff all three paths $r_{1}, r_{2}$, and $r$ have the same length $n$ and $\left(r_{1}^{i}, r_{2}^{i}\right) \triangleright^{G} r^{i}$ for all $i \in n$. This clearly makes $G^{\star}$ into a category with complementarity.

Let us now define our candidate complementarity structure on $\mathrm{fc}(G)$, for any $G \in$ GCompl. Let first $\mathrm{fc}(G)^{\mathbb{W}}$ be the image of $\left(G^{\star}\right)^{\mathbb{W}} \hookrightarrow G^{\star} \stackrel{\sim}{\rightarrow} \mathrm{fc}(G)$, i.e., all $i d$-free, closedworld paths. This in particular induces a functor $\left(G^{\star}\right)^{\mathbb{W}} \rightarrow(\mathrm{fc}(G))^{\mathbb{W}}$, with which $\ell^{\left(G^{\star}\right)}$ is obviously compatible, hence we define $\ell^{\mathrm{fc}(G)}$ to be the induced functor. Finally, let $\triangleright^{\mathrm{fc}(G)}$ be the following relational composite, where the backwards arrow denotes a converse:

$$
(\mathrm{fc}(G))^{2} \stackrel{(\widetilde{(})^{2}}{\longleftarrow}\left(G^{\star}\right)^{2} \stackrel{\triangleright^{G^{\star}}}{\longrightarrow}\left(G^{\star}\right)^{\mathbb{W}} \rightarrow \mathrm{fc}(G)^{\mathbb{W}} .
$$

Concretely, $\left(\rho_{1}, \rho_{2}\right) \nabla^{\mathrm{fc}(G)} \rho_{3}$ iff there exist $\left(r_{1}, r_{2}\right) \nabla^{G^{\star}} r_{3}$ such that $\widetilde{r_{i}}=\rho_{i}$ for $i=1,2,3$. Intuitively, $\rho_{1}$ and $\rho_{2}$ are coherent if upon insertion of identities at appropriate places they become pointwise coherent.

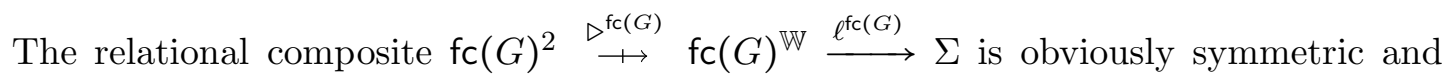
furthermore partially functional on objects, so in order to equip $\mathrm{fc}(G)$ with complementarity structure, it only misses partial functoriality on morphisms.

Definition 6.34. Let $\mathrm{GCompl}_{+}$denote the full subcategory of GCompl spanning objects $G$ such that the above composite is partially functorial on morphisms, which we call functorial graphs with complementarity.

Example 6.35. A sufficient condition for a graph with complementarity $G$ to be functorial is to satisfy

(i) for any two edges $e$ and $e^{\prime}$, and object $x$, if $e \subset e^{\prime}, e \neq i d$, and $e \smile i d_{x}$, then $e^{\prime}$ is an identity;

(ii) for all edges $e$ and $e^{\prime},\left(e \Downarrow i \overline{d) ;(i d} \Downarrow e^{\prime}\right)$ and $\left(i d \Downarrow \overline{\left.e^{\prime}\right) ;(e} \Downarrow i d\right)$ are defined at the same time and then equal.

The three graphs with complementarity $C C S, \mathcal{S}^{\mathbb{I Q}}$, and $\mathcal{T}^{\mathbb{I}}$ satisfy these conditions, hence are functorial.

The forgetful functor UCompl of course lands into $\mathrm{GCompl}_{+}$and we view it as a functor CCompl $\rightarrow \mathrm{GCompl}_{+}$from now on.

Proposition 6.36. The above construction of $\mathrm{fc}(G)^{\mathbb{W}}$, $\ell^{\mathrm{fc}(G)}$, and $\triangleright^{\mathrm{fc}(G)}$ extends to a left adjoint to UCompl, which coincides with fc on underlying graphs.

We henceforth denote the left adjoint by fc. 
Proof. Proving that this is left adjoint to UCompl reduces to showing that the composite

$$
\mathrm{GCompl}_{+}(G, \mathrm{UCompl}(C)) \hookrightarrow \mathrm{Gph}(G, U(C)) \stackrel{\cong}{\rightarrow} \mathrm{Cat}(\mathrm{fc}(G), C)
$$

factors through $\mathrm{CCompl}(\mathrm{fc}(G), C) \hookrightarrow \operatorname{Cat}(\mathrm{fc}(G), C)$, and conversely the composite

$$
\operatorname{CCompl}(\mathrm{fc}(G), C) \hookrightarrow \operatorname{Cat}(\mathrm{fc}(G), C) \stackrel{\cong}{\rightarrow} \mathrm{Gph}(G, U(C))
$$

factors through $\mathrm{GCompl}_{+}(G, \mathrm{UCompl}(C)) \hookrightarrow \mathrm{Gph}(G, U(C))$, which is routine.

We may now state the main property of blind composition:

Proposition 6.37. For any graphs with complementarity $G$ and $H$ over $A$, and transition sequences $x A \stackrel{\rho_{x}}{\Longleftarrow} x^{\prime}$ and $y A \stackrel{\rho_{y}}{\Longleftarrow} y^{\prime}$ respectively in $G$ and $H$, if $\left(\rho_{x}, \rho_{y}\right) \triangleright^{\mathrm{fc}(A)} \rho$, then

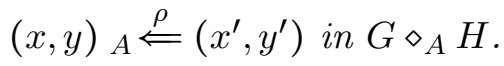

Proof. Let $p: G \rightarrow A$ and $q: H \rightarrow A$ be the given projections. Let also $\left(r_{1}^{i}, r_{2}^{i}\right) \triangleright^{A} r_{3}^{i}$ for all $i \in n$ witness the fact that $\left(\rho_{x}, \rho_{y}\right) \triangleright^{\mathrm{fc}(A)} \rho$. It is enough to prove $(x, y) \underset{A^{\star}}{\stackrel{r_{3}}{\longleftarrow}}\left(x^{\prime}, y^{\prime}\right)$, which is in fact a trivial induction on the length of $r_{3}$ using the definition of $G \diamond_{A} H$.

6.4. Trees. Returning to our main question, we know by Theorem 5.35 that the graph morphism $\mathcal{T} \rightarrow \mathcal{S}$ is a functional, strong bisimulation over $\mathbb{Q}$. Hence, by Proposition 2.11, we have:

Proposition 6.38. The graph morphism $\mathcal{T}^{\mathbb{Q}} \rightarrow \mathcal{S}^{\mathbb{I} Q}$ is a functional, strong bisimulation over $\mathbb{I} \mathbb{Q}$, and thus also over $\mathbb{A}$.

In this section, we introduce a criterion for a relation $R: G \mapsto H$ between modular graphs with complementarity over some adequate alphabet $A$, which essentially ensures that if $R \subseteq \approx A$, then $R$ is fair. This will reduce our main question to proving that the full relation induced by the map $C C S \hookrightarrow \mathcal{T}^{\mathbb{Q} Q}$ is included in weak bisimilarity over $\mathbb{A}$, which we do in Section 6.5.

Our criterion will rest upon the notion of $A$-tree, for any graph with complementarity $A$, which is directly inspired by the work of Brinksma et al. on failures [48].

Let the set $\mathcal{H}^{A}$ of $A$-trees consist of possibly infinite terms in the grammar

$$
\frac{\ldots \quad v_{i} \vdash t_{i} \quad \ldots \quad(\forall i \in n)}{v \vdash \sum_{i \in n} a_{i} \cdot t_{i}}(n \in \mathbb{N})
$$

where for all $i \in n, a_{i}: v_{i} \rightarrow v$ in $A$ is not silent, i.e., $a_{i} \in A^{\mathbb{W}}$ implies $\ell^{A}\left(a_{i}\right) \neq i d$. $A$-trees form a reflexive graph over $A$ with edges determined by

$$
\left(v \vdash \sum_{i \in n} a_{i} . t_{i}\right) \stackrel{a_{i}}{\longleftarrow}\left(v_{i} \vdash t_{i}\right) .
$$

Definition 6.39. A modular graph $p: G \rightarrow A$ over $A$ has enough $A$-trees iff for all $x \in G$, $v \in A$ such that $p(x) \frown v$, for all $A$-trees $v \vdash t$, there exists $x_{t} \in G$ such that $x \frown x_{t}$ and $x_{t}$ is weakly bisimilar to $t$ (over $A$ ).

Remark 6.40. In the case where $x \frown x^{\prime}$ iff $p(x) \frown p\left(x^{\prime}\right)$, this is equivalent to requiring that for all $a \in A$ and $A$-tree $t$ over $a$, there exists $x_{t} \in G$ such that $p\left(x_{t}\right)=a$ and $x_{t} \approx A t$.

Example 6.41. $C C S, \mathcal{S}^{\mathbb{I} Q}$, and $\mathcal{T}^{\mathbb{I}}$ have enough $\mathbb{A}$-trees, and Remark 6.40 applies. 
$A$-trees yield a new testing equivalence, called $A$-tree equivalence, as follows.

Definition 6.42. For any modular $p: G \rightarrow A$, let $\sim_{f}^{G \mid A}$ be the relation defined by $x \sim_{f}^{G \mid A} y$ iff $x \bowtie y$ and for all $v \in A$ such that $p(x) \frown v$ and $A$-trees $t \in \mathcal{H}_{v}^{A}$,

$$
(x, t) \in \perp^{G \diamond_{A} \mathcal{H}^{A}} \text { iff }(y, t) \in \perp^{G \diamond_{A} \mathcal{H}^{A}} \text {. }
$$

A graph with complementarity $A$ has enough ticks iff for all $a \in A$, there exists an edge $\nabla_{a}: a^{\prime} \rightarrow a$ such that $\ell^{A}\left(\nabla_{a}\right)=\varnothing$. Furthermore, $A$ is inertly silent iff for all $e: b \rightarrow a$ in $A^{\mathbb{W}}$ such that $\ell^{A}(e)=i d$, we have $a=b$ and $e=i d_{a}$.

Definition 6.43. A graph with complementarity $A$ is a nice alphabet iff it has enough ticks, and is finitely branching and inertly silent.

Example 6.44. $\mathbb{A}$ is a nice alphabet, but $\mathbb{I} \mathbb{Q}$ is not, because it is not inertly silent.

The main property of $A$-trees is:

Proposition 6.45. Consider any modular $G$ and adequate $p: G \rightarrow A$, where $G$ has enough A-trees and $A$ is a nice alphabet. Then, $\sim_{f}^{G}=\sim_{f}^{G \mid A}$.

We start with some preparation. Let a path in $A$ be loud iff it contains no silent (=identity if $A$ is inertly silent) edge, and $\nabla$-free iff no edge is in $\left(\ell^{A}\right)^{-1}(\varnothing)$. Let the set $\mathcal{F}_{a}$ of failures over $a \in A$ consist of all pairs $(p, L)$, where $p: a^{\prime} \rightarrow^{\star} a$ is any loud, $\diamond$-free path in $A$ and $L \subseteq A^{\star}$ is a set of loud paths such that for all $q \in L, \operatorname{cod}(q)=a^{\prime}$.

We define a map fl: $\mathcal{F}_{a} \rightarrow \mathcal{H}_{a}^{A}$ to $A$-trees over $a$, for all $a$, by induction on $p$, followed by coinduction on $L$ :

$$
\begin{aligned}
(e \circ p, L) & \mapsto e .(\mathbf{f l}(p, L))+\oslash_{a} .0 \\
(\epsilon, L) & \mapsto \mathbf{f}(L) \\
L & \mapsto \sum_{\{e \in A(-, a) \mid L \cdot e \neq \varnothing\}} e . \mathbf{f l}(L \cdot e)
\end{aligned}
$$

where $L \cdot e$ is the set of paths $p$ such that $(e \circ p) \in L$. Note in particular that if $L=\varnothing$ or $\{\epsilon\}$, then $\mathrm{fl}(L)=0$. The sum is finite at each stage because $A$ is finitely branching, and we use the fact that $A$ has enough ticks.

Proof of Proposition 6.45. It is straightforward to show that $\sim_{f}^{G} \subseteq \sim_{f}^{G \mid A}$, by Proposition 6.30. For the converse, assume $x \bowtie y$ and $x \psi_{f}^{G} y$. This means that there exists $z$ such that $x \circlearrowright z$ and $y \circlearrowright z$, and, w.l.o.g., $(y, z) \in \perp^{G \diamond_{A} G}$ and $(x, z) \notin \perp^{G \diamond_{A} G}$.

By the latter, we obtain a transition sequence $(x, z) \Leftarrow\left(x^{\prime}, z^{\prime}\right)$, such that for no $\left(x^{\prime \prime}, z^{\prime \prime}\right)$ we have $\left(x^{\prime}, z^{\prime}\right) \stackrel{\ominus}{=}\left(x^{\prime \prime}, z^{\prime \prime}\right)$. Let $r$ be the given path witnessing $(x, z) \Leftarrow\left(x^{\prime}, z^{\prime}\right)$. Its second projection $\left(\pi^{\prime}\right)^{\star}(r)$ is mapped by $p^{\star}$ to a path in $A$, from which we remove all identity edges (which are also all silent edges by $A$ being inertly silent) to obtain $\rho=\left(p \widetilde{\left.\circ \pi^{\prime}\right)^{\star}}(r)\right.$, a loud, $\odot$-free path in $A$. Further let $L \subseteq A^{\star}$ be the set of all $\overline{p^{\star}\left(r^{\prime}\right)}$ for paths $r^{\prime}: z^{\prime} \leftarrow^{\star} z^{\prime \prime}$. Let $t=\mathbf{f l}(\rho, L)$. We show $(x, t) \notin \perp^{G \diamond_{A} \mathcal{H}^{A}}$ and $(y, t) \in \perp^{G \diamond_{A} \mathcal{H}^{A}}$.

For the first point, $t \stackrel{\rho}{=} t^{\prime}$, with $t^{\prime}=\mathrm{fl}(\epsilon, L)$, hence $(x, t) \Leftarrow\left(x^{\prime}, t^{\prime}\right)$, by Proposition 6.37. Now, assume $\left(x^{\prime}, t^{\prime}\right) \stackrel{\ominus}{=} b^{\prime \prime}$. By definition of $G \diamond_{A} \mathcal{H}^{A}$, we split this into $x^{\prime} \stackrel{\rho_{1}}{=} x^{\prime \prime}$ and $t^{\prime} \stackrel{\rho_{2}}{=} t^{\prime \prime}$, with $b^{\prime \prime}=\left(x^{\prime \prime}, t^{\prime \prime}\right)$. But then $z^{\prime} \stackrel{\rho_{2}}{=} z^{\prime \prime}$ by construction of $t$, and hence $\left(x^{\prime}, z^{\prime}\right) \stackrel{\odot}{=}\left(x^{\prime \prime}, z^{\prime \prime}\right)$ (by Proposition 6.37), contradicting $(x, z) \notin \perp^{G \diamond_{A} G}$. 
Let us now show $(y, t) \in \perp^{G \diamond_{A} \mathcal{H}^{A}}$. For any $(y, t) \Leftarrow\left(y^{\prime}, t^{\prime}\right)$, we have accordingly $t \stackrel{\rho^{\prime}}{=} t^{\prime}$. By construction, $\rho^{\prime}$ is in the prefix closure of $\rho \circ L$,i.e.,

$$
\rho^{\prime} \in\left\{r \in A^{\star} \mid \exists r^{\prime} \in A^{\star}, l \in L, r \circ r^{\prime}=\rho \circ l\right\} .
$$

- If $\rho^{\prime}$ is a strict prefix of $\rho$, then by construction $t^{\prime} \stackrel{\ominus}{=} 0$ and we are done by Proposition 6.37, since $(i d, \nabla) \triangleright^{\mathbb{A}} \varnothing$.

- Otherwise, $\rho$ is a prefix of $\rho^{\prime}$. Let $\rho^{\prime \prime}$ be the unique path such that $\rho^{\prime}=\rho \circ \rho^{\prime \prime}$. We have $\rho^{\prime \prime} \in L$, hence by construction of $L$ there exists $z^{\prime \prime}$ such that $z \stackrel{\rho}{\Longleftrightarrow} z^{\prime} \stackrel{\rho^{\prime \prime}}{=} z^{\prime \prime}$, and thus $(y, z) \Leftarrow\left(y^{\prime}, z^{\prime \prime}\right)$, by Proposition 6.37. By $(y, z) \in \perp^{G \diamond_{A} G}$, there exists $\left(y^{\prime}, z^{\prime \prime}\right) \stackrel{\ominus}{\Longleftarrow}\left(y^{\prime \prime}, z^{\prime \prime \prime}\right)$, which projects to $y^{\prime} \stackrel{\rho_{y}}{=} y^{\prime \prime}$ and $z^{\prime \prime} \stackrel{\rho_{z}}{=} z^{\prime \prime \prime}$. But then $t^{\prime} \stackrel{\rho_{z}}{=} t^{\prime \prime}$, hence $\left(y^{\prime}, t^{\prime}\right) \stackrel{\wp}{\Longleftrightarrow}\left(y^{\prime \prime}, t^{\prime \prime}\right)$, by Proposition 6.37 again, which concludes the proof.

Corollary 6.46. For any nice alphabet $A$, modular $G$ and $H$, adequate $p: G \rightarrow A$ and q: $H \rightarrow A$, and relation $R: G \longrightarrow H$ over $A$ such that $R \subseteq \approx_{A}$, if $G$ and $H$ have enough $A$-trees and $R$ preserves and reflects $\bowtie$, then for any $x R x^{\prime}$ and $y R y^{\prime}$, we have $x \sim_{f}^{G} y$ iff $x^{\prime} \sim_{f}^{H} y^{\prime}$.

Proof. We have

$$
\begin{aligned}
& x \sim{ }_{f}^{G} y \\
& \Uparrow \text { (by Proposition 6.45) } \\
& x \bowtie y \text { and } \forall v \in p(x)^{\complement} \cdot \forall t \in \mathcal{H}_{v}^{A} .(x, t) \in \perp^{G \diamond_{A} \mathcal{H}^{A}} \Leftrightarrow(y, t) \in \perp^{G \diamond_{A} \mathcal{H}^{A}} \\
& \Uparrow \text { (by weak bisimilarity over } A \text { ) } \\
& x^{\prime} \bowtie y^{\prime} \text { and } \forall v \in p(x)^{\complement} . \forall t \in \mathcal{H}_{v}^{A} .\left(x^{\prime}, t\right) \in \perp^{H \diamond_{A} \mathcal{H}^{A}} \Leftrightarrow\left(y^{\prime}, t\right) \in \perp^{H \diamond_{A} \mathcal{H}^{A}} \\
& \mathbb{\Downarrow} \text { (by Proposition } 6.45 \text { again) } \\
& x^{\prime} \underset{f}{\sim} y^{\prime} .
\end{aligned}
$$

6.5. Main result. We now provide the missing piece to our main result, and then conclude.

Lemma 6.47. The graph of $\theta: \mathrm{ob} C C S \rightarrow \mathrm{ob} \mathcal{T}^{\mathbb{I Q}}$ is included in weak bisimilarity over $\mathbb{A}$.

Proof. We would like, for any $h: I \rightarrow X$ and family $P \in \prod_{n \in \mathbb{N}} \prod_{x \in X[n]} C C S_{n}$, to define a process term $h[P]$ with interface $I(\star)$, which would amount to

$$
\left(\left.\left.\right|_{n}\right|_{x \in X[n]} P_{x}\left[l \mapsto x \cdot s_{l}\right]\right),
$$

but restricting all channels in $X(\star) \backslash h(I(*))$. When $h$ is not an inclusion, this is a bit tricky, because in our De Bruijn-like syntax $\Gamma \vdash \nu . P$ may be understood as $\Gamma \vdash \nu(\Gamma+1)$. $P$. That is, $\nu$-bound channels are always strictly greater than names in $\Gamma$.

The correct way of doing this is to use subtraction, i.e., restrict channels in $X(\star)-I(\star)$, and accordingly rename channels in the body. Formally, let $\gamma_{h}$ be the unique non-decreasing isomorphism $(X(\star) \backslash h(I(\star))) \rightarrow(X(\star)-I(\star))$ (which exists thanks to $h$ being monic), and let $h[P]$ be

$$
I(\star) \vdash \nu^{X(\star)-I(\star)} \cdot\left(\left.l_{n}\right|_{x \in X[n]} P_{x}\left[\begin{array}{ll}
l \mapsto \epsilon a .\left(h_{\star}(a)=x \cdot s_{l}\right) & \text { if } x \cdot s_{l} \in h_{\star}(I(\star)) \\
l \mapsto \gamma_{h}\left(x \cdot s_{l}\right) & \text { otherwise }
\end{array}\right]\right),
$$

where $\epsilon$ is Hilbert's definite description operator, i.e., $\epsilon a . A(a)$ denotes the unique $a$ such that $A(a)$ holds, and $\nu^{n} . P$ denotes $\nu \ldots \nu . P, n$ times.

Definition 6.48. Let $\mathrm{J}$ : ob $C C S \longrightarrow \mathrm{ob} \mathcal{T}^{\mathbb{Q} Q}$ consist, for any $P \in \prod_{n \in \mathbb{N}} \prod_{x \in X[n]} C C S_{n}$, of all pairs $(h[P],(I, h, \theta(P))$. 
Let $\mathcal{R}$ be the composite relation

$$
\text { ob } C C S \stackrel{\equiv}{\stackrel{\equiv}{\longrightarrow}} \mathrm{ob} C C S \stackrel{\mathcal{J}}{\longrightarrow} \mathrm{ob} \mathcal{T}^{\mathbb{Q} Q} \text {. }
$$

We show that $\mathcal{R}$ is an expansion [51, Chapter 6], which implies that it is a weak bisimulation. Hence, since the graph of $\theta$ is included in $\mathcal{R}$, this entails the desired result. Let $x \stackrel{\widehat{\alpha}}{\longleftarrow} x^{\prime}$ iff

- either $\alpha$ is an identity and $x \stackrel{x}{=}$ in zero or one step,

- or $\alpha$ is not an identity and $x \stackrel{\alpha}{=} x^{\prime}$.

Recall:

Definition 6.49. $\mathcal{R}$ is an expansion iff for all $P \mathcal{R} T$,

- if $P \stackrel{\alpha}{\leftarrow} P^{\prime}$, then there exists $T^{\prime}$ such that $P^{\prime} \mathcal{R} T^{\prime}$ and $T \stackrel{\alpha}{\Longleftarrow} T^{\prime}$; and

- if $T \stackrel{\alpha}{\leftarrow} T^{\prime}$, then there exists $P^{\prime}$ such that $P \stackrel{\widehat{\alpha}}{\leftarrow} P^{\prime} \mathcal{R} T^{\prime}$.

First, one easily shows that transitions in $C C S$ are dealt with by 'heating' the right-hand side until it may match the given transition.

Conversely, we show below in (1) that for any transition $(I, h, \theta(P)) \stackrel{M}{\longleftarrow}\left(I, k, T^{\prime}\right)$, for $M: k \rightarrow h$ in $\mathbb{I} \mathbb{Q}$, where $M$ is either a fork or a channel creation, then $T^{\prime}=\theta\left(P^{\prime}\right)$, for some $P^{\prime} \in \prod_{n \in \mathbb{N}} \prod_{y \in Y[n]} C C S_{n}$, and $h[P] \equiv k\left[P^{\prime}\right]$.

Thus, any such transition, which is silent, is matched by the empty transition sequence, as in

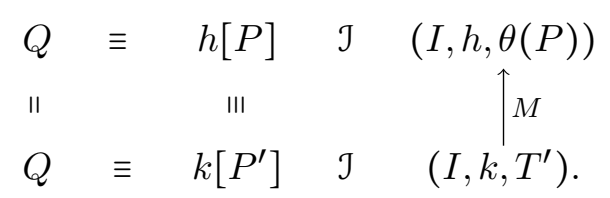

Similarly, for any transition $(I, h, \theta(P)) \stackrel{M}{\longleftarrow}\left(I, k, T^{\prime}\right)$ not falling in the previous cases, we prove below in (2) that there exists $P^{\prime} \in \prod_{n \in \mathbb{N}} \prod_{y \in Y[n]} C C S_{n}$ and $Q^{\prime}$ such that $h[P] \stackrel{\xi(M)}{\longleftarrow}$ $Q^{\prime} \equiv k\left[P^{\prime}\right]$. Thus, any such transition is matched as in

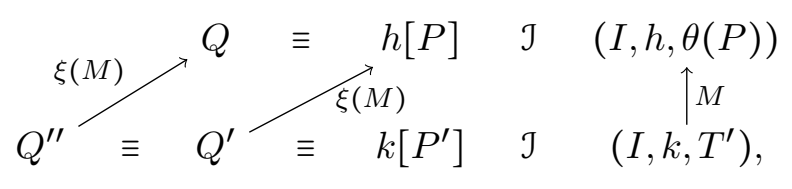

where $Q^{\prime \prime}$ is obtained by $\equiv$ being a bisimulation.

(1) As announced, let us now consider the case of a transition $(I, h, \theta(P)) \stackrel{M}{\longleftarrow}\left(I, k, T^{\prime}\right)$, for $M: k \rightarrow h$ in $\mathbb{I} \mathbb{Q}$, where $M$ is either a fork or a channel creation. Consider first the case where $M$ is a fork. Let $x_{1}, \ldots, x_{n}$ be the players of $X$, let $m_{1}, \ldots, m_{n}$ be their respective arities, and let $i_{0} \in n$ be the forking player. Let, for any $i \in n+1$,

$$
\mu(i)= \begin{cases}i & \text { if } i<i_{0} \\ i_{0} & \text { if } i=i_{0} \text { or } i=i_{0}+1 \\ i-1 & \text { if } i>i_{0}+1\end{cases}
$$


and

$$
P_{i}^{\prime}= \begin{cases}P_{i} & \text { if } i<i_{0} \\ P_{i_{0}}^{1} & \text { if } i=i_{0} \\ P_{i_{0}}^{2} & \text { if } i=i_{0}+1 \\ P_{i-1} & \text { if } i>i_{0}+1,\end{cases}
$$

where $P_{i_{0}}=P_{i_{0}}^{1} \mid P_{i_{0}}^{2}$. For all $j \in n+1$, we have that $y_{j}$ is an avatar of $x_{\mu(j)}$ (i.e., $\left.x_{\mu(j)}=\left(y_{j}\right)^{M}\right)$, and $P_{j}^{\prime}=P_{\mu(j)}$ if $\mu(j) \neq i_{0}$, while $P_{i_{0}}=P_{i_{0}}^{\prime} \mid P_{i_{0}+1}^{\prime}$.

Thanks to the restriction of edges

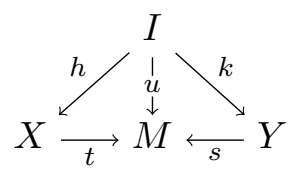

in $\mathbb{I} \mathbb{Q}$, for any $j \in n+1$, if $\mu(j)=i, l \in m_{i}$ and $a, b \in I(\star)$, we have that if $h_{\star}(a)=x_{i} \cdot s_{l}$ and $k_{\star}(b)=y_{j} \cdot s_{l}$, then, since $s \circ y_{j} \circ s_{l}=t \circ x_{i} \circ s_{l}$, both squares

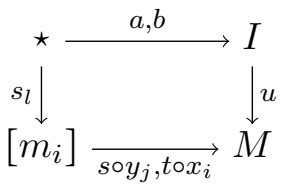

commute, hence $a=b$ by monicity of $u$.

So, for all $j \in n+1$ and $l \in m_{i}$, for $i=\mu(j)$, we have $x_{i} \cdot s_{l} \in h_{\star}(I(\star))$ iff $y_{j} \cdot s_{l} \in k_{\star}(I(\star))$, in which case

$$
\epsilon a .\left(h_{\star}(a)=x_{i} \cdot s_{l}\right)=\epsilon b \cdot\left(k_{\star}(b)=y_{j} \cdot s_{l}\right) .
$$

Furthermore, we have a commuting diagram

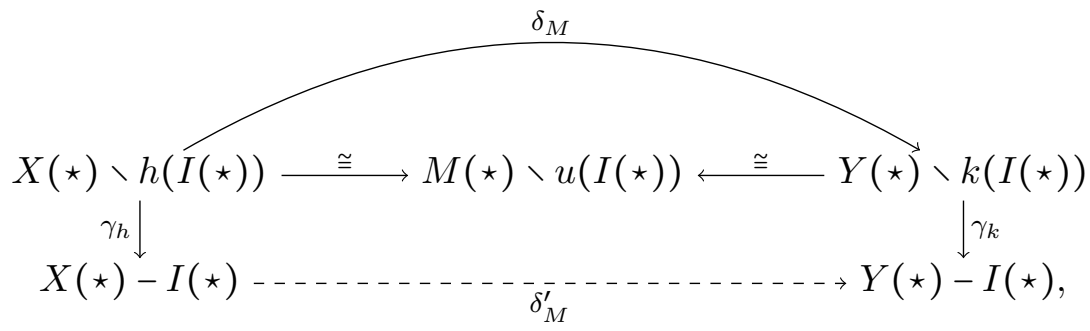

of bijections, where $\delta_{M}$ and $\delta_{M}^{\prime}$ are obtained by composition and the arrows marked $\cong$ are the respective restrictions of $t$ and $s$. This diagram is such that for all $j \in n+1$ and $i=\mu(j)$, $l \in m_{i}$, if $x_{i} \cdot s_{l} \notin h(I(\star))$, then $\delta_{M}\left(x_{i} \cdot s_{l}\right)=y_{j} \cdot s_{l}$. We have

$$
h[P]=\nu^{X(\star)-I(\star)} \cdot\left(\left.\right|_{i \in n} P_{i}\left[\begin{array}{ll}
l \mapsto \epsilon a .\left(h_{\star}(a)=x_{i} \cdot s_{l}\right) & \text { if } x_{i} \cdot s_{l} \in h_{\star}(I(\star)) \\
l \mapsto \gamma_{h}\left(x_{i} \cdot s_{l}\right) & \text { otherwise }
\end{array}\right]\right)
$$

and

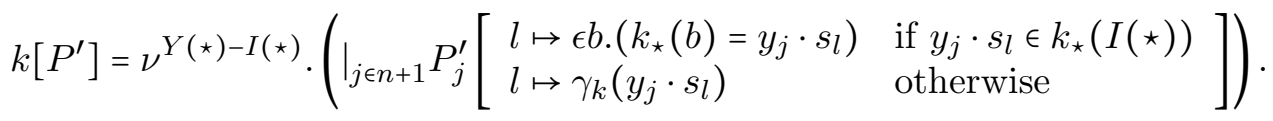


Via the renaming $\delta_{M}^{\prime}$, we have

$$
\begin{aligned}
& \begin{aligned}
h[P] & \equiv \nu^{Y(\star)-I(\star)} \cdot\left(l_{j \in n+1, j \neq i_{0}+1} P_{\mu(j)}\left[\begin{array}{ll}
l \mapsto \epsilon b \cdot\left(k_{\star}(b)=y_{j} \cdot s_{l}\right) & \text { if } y_{j} \cdot s_{l} \in k_{\star}(I(\star)) \\
l \mapsto \gamma_{k}\left(\delta_{M}\left(x_{i} \cdot s_{l}\right)\right) & \text { otherwise } \\
l \mapsto \epsilon b .\left(k_{\star}(b)=y_{j} \cdot s_{l}\right) & \text { if } y_{j} \cdot s_{l} \in k_{\star}(I(\star)) \\
\left.l \mapsto \gamma_{k}\left(y_{j} \cdot s_{l}\right)\right) & \text { otherwise }
\end{array}\right]\right)
\end{aligned} \\
& \stackrel{i d}{\longleftarrow} \nu^{Y(\star)-I(\star)} \cdot\left(\left.\right|_{j \in n+1} P_{j}^{\prime}\left[\begin{array}{ll}
l \mapsto \epsilon b .\left(k_{\star}(b)=y_{j} \cdot s_{l}\right) & \text { if } y_{j} \cdot s_{l} \in k_{\star}(I(\star)) \\
\left.l \mapsto \gamma_{k}\left(y_{j} \cdot s_{l}\right)\right) & \text { otherwise }
\end{array}\right]\right) \\
& \equiv k\left[P^{\prime}\right] .
\end{aligned}
$$

The case of a channel creation move is similar.

(2) Consider now any transition $(I, h, \theta(P)) \stackrel{M}{\longleftarrow}\left(I, k, T^{\prime}\right)$, where $M$ is an input or an output on some channel $c \in h_{\star}(I(\star))$, or a synchronisation, or a tick. Then, proceeding as for the forking move above, we may take $\mu=i d$, and still obtain $\delta_{M}$ and $\delta_{M}^{\prime}$. In all cases, we have $T_{i}^{\prime}=\theta\left(P_{i}^{\prime}\right)$, for some family $P^{\prime}$ of CCS processes. E.g., if $M$ is an input on $c$ by $x_{i_{0}}$, then $P_{i}^{\prime}=P_{i}$ for all $i \neq i_{0}$, and $P_{i_{0}} \equiv c . P_{i_{0}}^{\prime}+P^{\prime \prime}$. We have $h[P] \stackrel{\xi(M)}{\longleftarrow} Q$, where

$$
Q=\nu^{X(\star)-I(\star)} \cdot\left(\left.\right|_{i \in n} P_{i}^{\prime}\left[\begin{array}{ll}
l \mapsto \epsilon a .\left(h_{\star}(a)=x_{i} \cdot s_{l}\right) & \text { if } x_{i} \cdot s_{l} \in h_{\star}(I(\star)) \\
l \mapsto \gamma_{h}\left(x_{i} \cdot s_{l}\right) & \text { otherwise }
\end{array}\right]\right),
$$

which via the renaming $\delta_{M}^{\prime}$, is structurally congruent to

$$
\begin{aligned}
& \nu^{Y(\star)-I(\star)} \cdot\left(\left.\right|_{i \in n} P_{i}^{\prime}\left[\begin{array}{ll}
l \mapsto \epsilon b \cdot\left(k_{\star}(b)=y_{i} \cdot s_{l}\right) & \text { if } y_{i} \cdot s_{l} \in k_{\star}(I(\star)) \\
l \mapsto \gamma_{k}\left(\delta_{M}\left(x_{i} \cdot s_{l}\right)\right) & \text { otherwise }
\end{array}\right]\right) \\
& \equiv \nu^{Y(\star)-I(\star)} \cdot\left(\left.\right|_{i \in n} P_{i}^{\prime}\left[\begin{array}{ll}
l \mapsto \epsilon b .\left(k_{\star}(b)=y_{i} \cdot s_{l}\right) & \text { if } y_{i} \cdot s_{l} \in k_{\star}(I(\star)) \\
l \mapsto \gamma_{k}\left(y_{i} \cdot s_{l}\right) & \text { otherwise }
\end{array}\right]\right) \\
& \equiv k\left[P^{\prime}\right],
\end{aligned}
$$

which concludes the proof.

This leads to our first full abstraction result:

Corollary 6.50. The composite $\mathrm{ob}(C C S) \hookrightarrow \mathrm{ob}\left(\mathcal{T}^{\mathbb{Q Q}}\right) \rightarrow \mathrm{ob}\left(\mathcal{S}^{\mathbb{I Q}}\right)$ is included in weak bisimilarity.

Proof. By the previous lemma, Proposition 6.38, and the fact that weak bisimulations are closed under composition.

Corollary 6.51. The composite ob CCS $\stackrel{\theta}{\rightarrow}$ ob $\mathcal{T}^{\mathbb{I Q}} \stackrel{\llbracket-\rrbracket}{\longrightarrow}$ ob $\mathcal{S}^{\mathbb{I Q}}$ is fair, and we have for all CCS processes $P$ and $Q$ over any common $n$ :

$$
P \sim_{f, s} Q \quad \text { iff } \quad \llbracket \theta(P) \rrbracket \sim_{f} \llbracket \theta(Q) \rrbracket .
$$

Proof. We have:

$$
\begin{aligned}
& P \sim_{f, s} Q \\
& \mathbb{\Downarrow} \text { (by Proposition 6.21) } \\
& P \sim \underset{f}{C C S} Q \\
& \Uparrow \text { (by Corollaries 6.46 and 6.50, and Example 6.41) } \\
& \llbracket \theta(P) \rrbracket \sim_{f}^{\mathbb{S} \mathbb{Q}} \llbracket \theta(Q) \rrbracket \\
& \Uparrow \text { (by Corollary 6.26) } \\
& \llbracket \theta(P) \rrbracket \sim f \llbracket \theta(Q) \rrbracket,
\end{aligned}
$$


as desired.

\section{CCS AS A PLAYGROUND}

At last, we prove that $\mathbb{D}^{C C S}$ forms a playground. We rewind to the beginning of Section 4.1 , to state things a bit more formally.

7.1. A pseudo double category. Recall from $\mathrm{HP}$ the notion of dimension in $\mathbb{C}: \star$ is the sole object of dimension 0 , all $[n]$ 's have dimension 1 , all $o_{n, i}, \iota_{n, i}, \pi_{n}^{l}, \pi_{n}^{r}, \odot_{n}$, and $\nu_{n}$ have dimension 2 , all $\pi_{n}$ have dimension 3 , and all $\tau_{n, i, m, j}$ have dimension 4 . By extension, a presheaf $F$ has dimension $i$ if $F$ is empty over objects of dimension strictly greater than $i$. We call interfaces the presheaves of dimension 0 (i.e., empty beyond dimension 0 ), positions the finite presheaves of dimension 1.

We start by viewing the base pseudo double category of our playground, $\mathbb{D}^{C C S}$, as a sub-pseudo double category of the following pseudo double category $\mathbb{D}^{C C S, 0}$.

Definition 7.1. Let $\mathbb{D}^{C C S, 0}$ have:

- as objects all positions,

- horizontal category $\mathbb{D}_{h}^{C C S, 0}$ the subcategory of $\widehat{\mathbb{C}}^{f}$ consisting of positions and monic arrows between them;

- vertical (bi)category $\mathbb{D}_{v}^{C C S, 0}$ the sub-bicategory of Cospan $\left(\widehat{\mathbb{C}}^{f}\right)$ consisting of positions and cospans of monic arrows between them;

- and all commuting diagrams

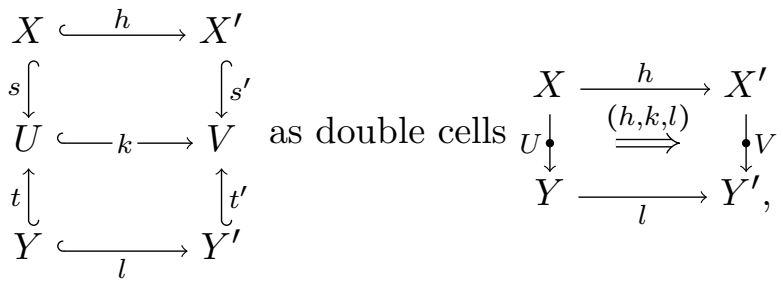

with all $\hookrightarrow$ arrows monic.

Horizontal composition of double cells is induced by composition in $\widehat{\mathbb{C}}^{f}$. Vertical composition of double cells is induced by pushout in $\widehat{\mathbb{C}}^{f}$. It is of course the vertical direction here which is pseudo.

Proposition 7.2. $\mathbb{D}^{C C S}$ is the pseudo double category obtained by restricting $\mathbb{D}^{C C S, 0}$ to vertical morphisms which are either equivalences or finite composites of moves.

Since $\mathbb{D}^{C C S}$ is again the only involved (candidate) playground in this section, we often omit the superscript. E.g., $\mathbb{D}^{0}$ denotes $\mathbb{D}^{C C S, 0}$.

The rest of Section 7 is devoted to proving:

Theorem 7.3. $\mathbb{D}$, equipped with

- as individuals, all positions of the shape $\mathbb{C}(-,[n])$, i.e., all strictly representable presheaves,

- moves as moves, seeds as basic moves, and full moves as full moves.

forms a playground. 
We start with a combinatorial correctness criterion for characterising plays $U: X \rightarrow Y$ among general cospans $X \hookrightarrow U \hookleftarrow Y$, which we then put to use in proving the theorem. Our convention for plays $X \hookrightarrow U \hookleftarrow Y$ is that the (candidate) final position is always on the left.

7.2. Correctness. We prove a few properties of plays, which we then find are sufficient for a cospan to be a play.

Given a play $X \hookrightarrow U \hookleftarrow Y$, we start by forgetting the cospan structure and exhibiting some properties of $U$ alone.

Definition 7.4. A core of a presheaf $U \in \widehat{\mathbb{C}}^{f}$ is an element of dimension $>1$ which is not the image (under the action of some morphism of $\mathbb{C}$ ) of any element of higher dimension.

Here is a first easy property of plays. Observing that for all seeds $Y \hookrightarrow M \hookleftarrow X, M$ is a representable presheaf, we put:

Definition 7.5. A presheaf $U$ is locally 1-injective iff for any seed $Y \hookrightarrow M \hookleftarrow X$ with interface $I$ and core $\mu \in U(M)$, if two elements of $M$ are identified by the Yoneda morphism $\mu: M \rightarrow U$, then they are in (the image of) $I(\star)$.

The name 'locally 1-injective' is designed to evoke the fact that $M \rightarrow U$ is injective above dimension 0 .

Proposition 7.6. Any play $U$ is locally 1-injective.

Proof. Choose a decomposition of $U$ into moves; $\mu$ corresponds to precisely one such move, say $M^{\prime}$, obtained, by definition, from some seed $M$ as a pushout (3.1). By construction of pushouts in presheaf categories, $M^{\prime}$ is obtained from $M$ by identifying some channels according to $I \rightarrow Z$.

We now extract from any presheaf a graph, which represents its candidate causal structure. Observe that, in $\mathbb{C}$, for any object $\mu$ of dimension $>1$ (i.e., a move), all morphisms from a player, i.e., an object of the shape $[n]$, to $\mu$ have exactly one of the shapes $f \circ s \circ f^{\prime}$ and $f \circ t \circ f^{\prime}$. In the former case, the given player belongs in the final position of $\mu$ and we say that it is a source of $\mu$; in the latter, it belongs in the initial position and we call it a target. We extend these notions to arbitrary presheaves.

Definition 7.7. In any $U$, the sources of a core $\mu$ are the players $x$ with a morphism, in $\int U$ (the category of elements of $U$, recalled in Section [3.1), of the shape $x \stackrel{f \circ s \circ f^{\prime}}{\longrightarrow} \mu$ to $\mu$; its targets are the players $y$ with a morphism of the shape $y \stackrel{f \circ t \circ f^{\prime}}{\longrightarrow} \mu$.

Example 7.8. In the representable $\pi_{n}$, there is one target, $l \circ t$ (or equivalently $r \circ t$ ), and two sources, $s_{1}=l \circ s$ and $s_{2}=r \circ s$, respectively the left and right players obtained by forking. Another example is $\tau_{n, i, m, j}$, which has two targets, the sender $\epsilon \circ t$ and the receiver $\rho \circ t$, and two sources $\epsilon \circ s$ and $\rho \circ s$.

Definition 7.9. A channel $a \in M(\star)$ is created by a seed $Y \stackrel{s}{\longrightarrow} M \stackrel{t}{\hookrightarrow} X$ iff $a \in Y(\star) \backslash X(\star)$.

Recall that in $\mathbb{C}$, the channels known to a player $[n]$ are represented by morphisms $s_{1}, \ldots, s_{n}: \star \rightarrow[n]$, so that in a presheaf $U \in \widehat{\mathbb{C}}^{f}$, the channels known to $x \in U[n]$ are $x \cdot s_{1}$, $\ldots$, and $x \cdot s_{n}$.

Given a presheaf $U$, we construct its causal (simple) graph $G_{U}$ as follows: 
- its vertices are all channels, players, and cores in $U$;

- there is an edge to each core from its sources and one from each core to its targets, as in

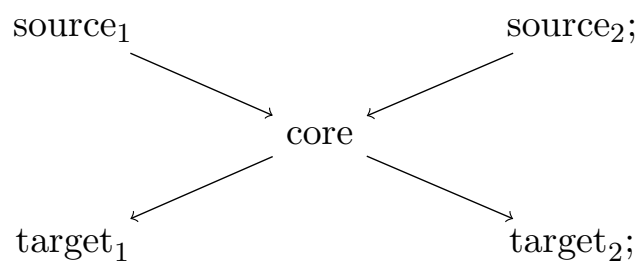

- there is an edge $x \rightarrow x \cdot s_{i}$ for all $x \in U[n]$ and $i \in n$;

- there is an edge $a \rightarrow \mu$ for each channel $a$ created by $\mu$.

This graph is actually a binary relation, since there is at most one edge between any two vertices. It is also a coloured graph, in the sense that it comes equipped with a morphism to the graph $L$ :

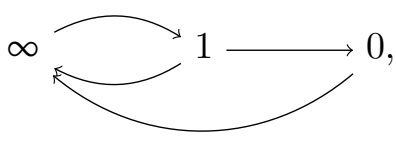

mapping cores to $\infty$, players to 1 , and channels to 0 . (Observe in particular that there are no edges from channels to players nor from cores to channels.) For any simple graph $G$, equipped with a morphism $l: G \rightarrow L$, we call vertices of $G$ channels, players, or cores, according to their label.

Definition 7.10. Seen as an object of $\mathrm{Gph} / L, G$ is source-linear iff for any cores $\mu, \mu^{\prime}$, and other vertex (necessarily a player or a channel) $x, \mu \leftarrow x \rightarrow \mu^{\prime}$ in $G$, then $\mu=\mu^{\prime}$. $G$ is target-linear iff for any cores $\mu, \mu^{\prime}$ and player $x$, if $\mu \rightarrow x \leftarrow \mu^{\prime}$ in $G$, then $\mu=\mu^{\prime}$. $G$ is linear iff it is both source-linear and target-linear.

Proposition 7.11. For any play $Y \stackrel{s}{\rightarrow} U \stackrel{t}{\rightarrow} X, G_{U}$ is linear.

Proof. By induction on any decomposition of $U$ into moves.

Proposition 7.12. For any play as above, $G_{U}$ is acyclic (in the directed sense).

Proof. Again by induction on any decomposition of $U$.

Definition 7.13. A player $x$ in $U$ is final iff it is not the target of any move, i.e., for no move $\mu \in U, x=\mu \cdot t$.

Lemma 7.14. A player is final in $U$ iff it has no edge from any core in $G_{U}$.

Definition 7.15. A player is initial in $U$ when it is not the source of any move, i.e., for no move $\mu \in U, x=\mu \cdot s$. A channel is initial when it is not created by any move.

Lemma 7.16. A player is initial in $U$ iff it has no edge to any core in $G_{U}$.

Now, here is the expected characterisation:

Theorem 7.17. A cospan $Y \stackrel{s}{\hookrightarrow} U \stackrel{t}{\longrightarrow} X$ is a play iff

(i) $U$ is locally 1-injective,

(ii) $X$ contains precisely the initial players and channels in $U$,

(iii) $Y$ contains all channels, plus precisely the final players in $U$, 
(iv) and $G_{U}$ is linear and acyclic.

Of course, we have almost proved the 'only if' direction, and the rest is easy, so only the 'if' direction remains to prove. The rest of this section is devoted to this. First, let us familiarise ourselves with removing elements from a presheaf. For two morphisms of

presheaves $U \stackrel{f}{\rightarrow} V \stackrel{g}{\leftarrow} W$, we denote by $U \backslash W$ the topos-theoretic difference $U \cap \neg W$ of (the images of) $f$ and $g$ in the lattice $\operatorname{Sub}(V)$ of subobjects of $V$. This differs in general from what we denote $U-W$, which is the set of elements in $V$ which are in the image of $U$ but not that of $W$, i.e., $\sum_{c \in \mathbb{C}} U(c) \backslash W(c)$. More generally, for any morphism of presheaves $f: U \rightarrow V$ and set $W$, let $U-W=\sum_{c \in \mathbb{C}} \operatorname{Im}(U(c)) \backslash W$. $U-W$ is generally just a set, not a presheaf; i.e., its elements are not necessarily stable under the action of morphisms in $\mathbb{C}$. Proposition 7.19 below exhibits a case where they are, which is useful to us.

Definition 7.18. For any seed $Y \hookrightarrow M \hookleftarrow X$, let the past past $(M)=M-Y$ of $M$ be the set of its elements not in the image of $Y$. For any such $M$, presheaf $U$, and core $\mu \in U(M)$, let $\operatorname{past}(\mu)=\operatorname{Im}(\operatorname{past}(M))$ consist of all images of past $(M)$.

To explain the statement a bit more, by Yoneda, we see $\mu$ as a map $M \rightarrow U$, so we have a set-function

$$
\operatorname{past}(M) \hookrightarrow \int M \rightarrow \int U
$$

Observe that past $(\mu)$ is always a set of players and moves only, since channels present in $X$ always are in $Y$ too.

Given a core $\mu \in U$, an important operation for us will be

$$
U \nabla \mu=\bigcup\left\{V \hookrightarrow U \mid \int V \cap \operatorname{past}(\mu)=\varnothing\right\} .
$$

$U \rrbracket \mu$ is thus the largest subpresheaf of $U$ not containing any element of the past of $\mu$. The good property of this operation is:

Proposition 7.19. If $\mu$ is a maximal core in $G_{U}$ (i.e., there is no path to any further core) and $G_{U}$ is target-linear, then $U \nabla \mu=U-\operatorname{past}(\mu)$, i.e., $(U \nabla \mu)(c)=U(c) \backslash \operatorname{past}(\mu)$ for all $c$.

Proof. The direction $(U \nabla \mu)(c) \subseteq U(c) \backslash \operatorname{past}(\mu)$ is by definition of $\square$. Conversely, it is enough to show that $c \mapsto U(c) \backslash$ past $(\mu)$ forms a subpresheaf of $U$, i.e., that for any $f: c \rightarrow c^{\prime}$ in $\mathbb{C}$, and $x \in U\left(c^{\prime}\right) \backslash \operatorname{past}(\mu), x \cdot f \notin \operatorname{past}(\mu)$. Assume on the contrary that $x^{\prime}=x \cdot f \in \operatorname{past}(\mu)$. Then, of course $f$ cannot be the identity. Furthermore, $x^{\prime}$ is either a player or a move; so, up to pre-composition of $f$ with a further morphism, we may assume that $x^{\prime}$ is a player. But then, since $f$ is non-identity, $x$ must be a move, with $x^{\prime}$ being one of its sources or targets. Now, up to post-composition of $f$ with a further morphism, we may assume that $x$ is a core. So, there is either an edge $x \rightarrow x^{\prime}$ or an edge $x^{\prime} \rightarrow x$ in $G_{U}$. However, $x \neq \mu$, so $x \rightarrow x^{\prime}$ is impossible by target-linearity of $G_{U}$, and $x^{\prime} \rightarrow x$ is impossible by maximality of $\mu$.

Proof of Theorem 7.17. We proceed by induction on the number of moves in $U$. If it is zero, then $U$ is a position; by (ii), $t$ is an iso, and by (iii)] so is $s$, hence the cospan is a play. For the induction step, we first decompose $U$ into

$$
Y \stackrel{s_{2}}{\longrightarrow} U^{\prime} \stackrel{t_{2}}{\longrightarrow} Z \stackrel{s_{1}}{\longrightarrow} M^{\prime} \stackrel{t_{1}}{\longrightarrow} X,
$$

and then show that $M^{\prime}$ is a move and $U^{\prime}$ satisfies the conditions of the theorem.

So, first, pick a maximal core $\mu$ in $G_{U}$, i.e., one with no path to any other core. Let 


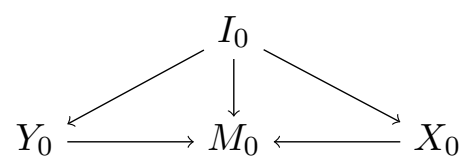

be the seed with interface corresponding to $\mu$, so we have the Yoneda morphism $\mu: M_{0} \rightarrow U$.

Let $U^{\prime}=(U \nabla \mu)$, and $X_{1}=X-\operatorname{Pl}\left(X_{0}\right) . X_{1}$ is a subpresheaf of $X$, since it contains all names. The square

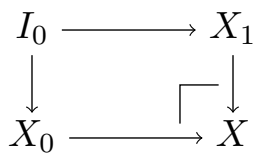

is a pushout, since it just adds the missing players to $X_{1}$. Define now $Z, M^{\prime}, s_{1}$, and $t_{1}$ by the pushouts

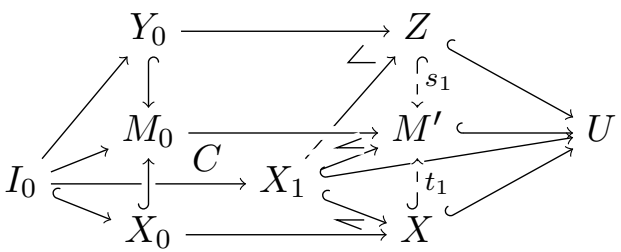

and the induced arrows. We further obtain arrows to $U$ by universal property of pushout, which are monic because $X \hookrightarrow U$ is, using (i). We observe that $U=M^{\prime} \cup U^{\prime}$, i.e., the square

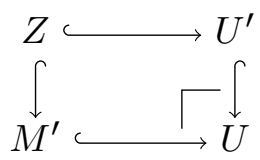

is a pushout, so $U$ is indeed a composite as claimed, with $Z \leftrightarrow M^{\prime} \leftrightarrow X$ a move by construction. So, it remains to prove that $Y \leftrightarrow U^{\prime} \leftrightarrow Z$ satisfies the conditions. First, as a subpresheaf of $U, U^{\prime}$ is locally 1-injective and has a linear and acyclic causal graph, so satisfies (i) and (iv). $U^{\prime}$ furthermore satisfies (ii) by construction of $Z$ and source-linearity of $G_{U}$, and (iii) because removing past $(\mu)$ cannot make any non-final player final.

\subsection{CCS as a pre-playground. We now start proving:}

Theorem 7.20. $\mathbb{D}$ forms a playground.

Axioms (P2) (P4) are easy, as well as (P6), (P9) and (P10) Furthermore, once (P1) is clear, (P5) is also easy. This leaves (P1) and the decomposition axioms.

For (P1), i.e., the fact that cod: $D \rightarrow \mathbb{D}_{h}$ is a fibration, we introduce the notion of 'history' for plays. For a presheaf $U \in \widehat{\mathbb{C}}^{f}$, let ${ }_{\llcorner} U$, be its restriction to dimension 3, i.e., ${ }_{\llcorner} U_{\lrcorner}\left(\tau_{n, i, m, j}\right)=\varnothing$ for all $n, i, m, j$, and ${ }_{\llcorner} U_{\lrcorner}(c)=U(c)$ on other objects. Further let $\operatorname{El}(U)=$ $\sum_{c \in \mathrm{ob}(\mathbb{C})_{L}} U_{\lrcorner}(c)$ be the set of elements of ${ }_{\llcorner} U_{\lrcorner}$. We have a category $\operatorname{El}\left(\widehat{\mathbb{C}}^{f}\right)$, whose objects are those of $\widehat{\mathbb{C}}^{f}$, and whose morphisms $U \rightarrow U^{\prime}$ are set-functions $\operatorname{El}(U) \rightarrow \operatorname{El}\left(U^{\prime}\right)$. We denote such morphisms with special arrows $U \rightarrow U^{\prime}$. There is a forgetful functor $\mathrm{El}: \widehat{\mathbb{C}}^{f} \rightarrow \operatorname{El}\left(\widehat{\mathbb{C}}^{f}\right)$, which we implicitly use in casting arrows $U \rightarrow U^{\prime}$ to arrows $U \rightarrow U^{\prime}$. 
Definition 7.21. Consider any seed $X \hookrightarrow M \hookleftarrow Y$ which is not a synchronisation, where $Y$ is the initial position and $X$ is the final position. Then $Y$ is a representable position, say $[n]$, and we let the history of $M$ be the $\operatorname{map} p_{M}: \operatorname{El}(M) \rightarrow \operatorname{El}(Y)$ sending

- all channels in $\operatorname{El}(M) \cap \operatorname{El}(Y)$ to themselves,

- all other elements to $i d_{[n]}$.

The history $p_{M^{\prime}}$ of a move $M^{\prime}$ is the map obtained by pushout of the history of its generating seed $M$, as in

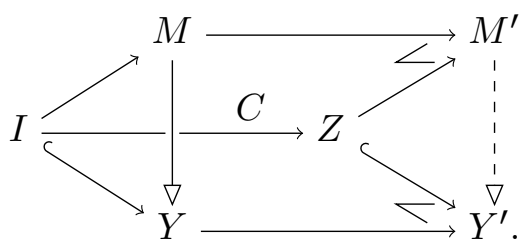

This defines the history of moves. We have:

Proposition 7.22. For any move $X \stackrel{s}{\rightarrow} M \stackrel{t}{\rightarrow} Y$, we have $p_{M} \circ t=i d$.

We graphically represent histories by arrows between the presheaves, as $p$ in

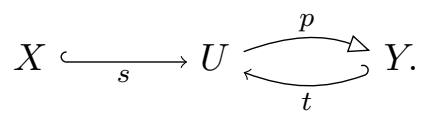

We now define the history of sequences of moves, which we here call sequential plays. We denote such a sequence $X_{n} \stackrel{M_{n}}{\longrightarrow} X_{n-1} \ldots X_{1} \stackrel{M_{1}}{\longrightarrow} X_{0}$ by $\left(M_{n}, \ldots, M_{1}\right)$.

Definition 7.23. Define now the history of a sequential play $X \rightarrow\left(M_{n}, \ldots, M_{1}\right) \leftarrow Y$, letting $U=M_{1} \bullet \ldots \bullet M_{n}$ be the corresponding play, to be the map $U \rightarrow Y$ defined by induction on $n$ as follows:

- if $|U|=0$, then $t$ is an isomorphism, and the history is the inverse of the corresponding bijection on elements;

- if $|U|=1$, then $U$ is a move $M$ and its history is that of $M$;

- if $|U|>1$, then $U=\left(U^{\prime}, M\right)$ for some move $M$ and sequential play $U^{\prime}$; letting $p_{U^{\prime}}$ be the history of $U^{\prime}$ obtained by induction hypothesis, we let $p_{U}=p_{M} \circ q$, where $q$ is defined by universal property of pushout in

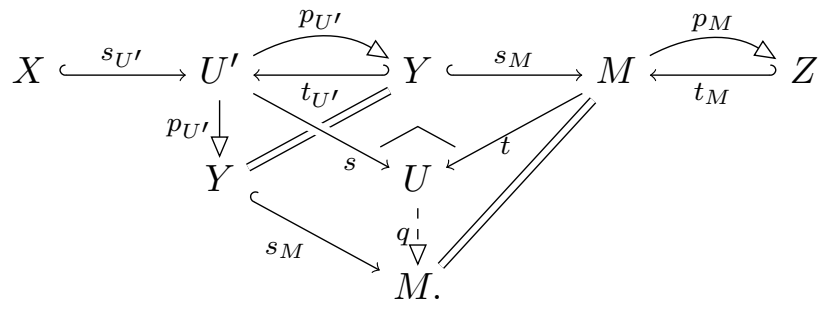

Proposition 7.24. For any sequential plays $U_{1}, U_{2}: X \rightarrow Y$ with isomorphic compositions, we have $p_{U_{1}}=p_{U_{2}}$.

Proof. For any presheaf $U$ such that $G_{U}$ is source-linear and acyclic, consider the function $h_{U}: \operatorname{El}(U) \rightarrow \operatorname{El}(U)$ mapping

- initial players and channels to themselves, 
- non-initial players and channels to the (unique by source-linearity of $G_{U}$ ) core that created them,

- elements of dimension 2 to their image under $t$,

- elements of higher dimensions to the image of one of their images in dimension 2 (which all map to the same element by a simple case analysis).

Observe that this map is ultimately idempotent because it is strictly increasing w.r.t. $G_{U}$, and let $H_{U}$ be the corresponding idempotent function.

It is easy to see that if $X \hookrightarrow U \hookleftarrow Y$ is a move, then $\operatorname{Im}\left(H_{U}\right)=Y$ and $p_{U}=H_{U}$.

Furthermore, for all composable plays $X \stackrel{U^{\prime}}{\rightarrow} Y \stackrel{U}{\rightarrow} Z$, we have $H_{U} \cdot U^{\prime}=H_{U} \circ H_{U^{\prime}}^{U}$, where $H_{U^{\prime}}^{U}: \operatorname{El}\left(U \bullet U^{\prime}\right) \rightarrow \operatorname{El}(U)$ is the extension of $H_{U^{\prime}}$ to $\operatorname{El}\left(U \bullet U^{\prime}\right)$ which is the identity on $\operatorname{El}(U) \backslash \operatorname{El}\left(U^{\prime}\right)$. Because $\operatorname{Im}\left(H_{U^{\prime}}\right)=Y$, this indeed goes to $\operatorname{El}(U)$.

When $U$ is a move, this is actually equivalent to the diagrammatic definition of $p_{M \bullet U^{\prime}}$, which entails by induction that for any play $U, p_{U}=H_{U}$, which does not depend on the decomposition of $U$ into moves.

Just as for moves, the target map is a section of the history:

Proposition 7.25. For any play $X \hookrightarrow U \stackrel{t}{\rightarrow} Y$, we have $p_{U} \circ t=i d_{Y}$.

Proposition 7.26. Any double cell $(h, k, l)$ as on the left below
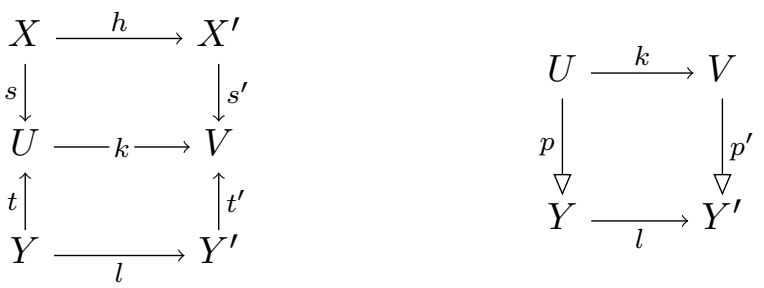

is compatible with histories $p: U \rightarrow Y$ and $p^{\prime}: U^{\prime} \rightarrow Y^{\prime}$, in the sense that the square on the right commutes.

The important point for us is:

Proposition 7.27. The vertical codomain functor cod: $Ð \rightarrow \mathbb{D}_{h}$ is a fibration.

Proof. We first consider the restriction of cod to the full subcategory of $D$ consisting of moves and isomorphisms. Given a move $X \stackrel{s}{\rightarrow} M \stackrel{t}{\rightarrow} Y$ and a morphism $l: Y^{\prime} \rightarrow Y$ in $\mathbb{D}_{h}$, consider the pullback (in sets) and the induced arrow $t^{\prime}$ :

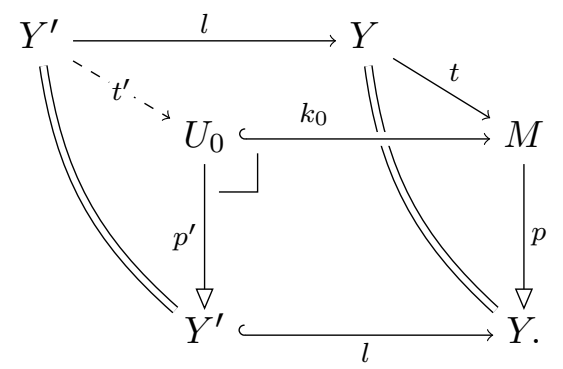

Now, consider $U_{0}$ as a presheaf over $\mathbb{C}_{3}$ by giving each element the type of its image under $k_{0}$, and checking that $U_{0}$, viewed as an ob( $\left.\mathbb{C}_{3}\right)$-indexed family of subsets of $M$, is stable 
under the action of morphisms in $\mathbb{C}_{3}$. This, in passing, equips $k_{0}$ and $t^{\prime}$ with the structure of maps in $\widehat{\mathbb{C}}^{f}$.

Furthermore, let the $(n, i, m, j)$-horn (see, e.g., Joyal and Tierney [29] for the origin of our terminology) $\tau_{n, i, m, j}^{-}$be the representable presheaf on $\tau_{n, i, m, j}$, minus the element $i d_{\tau_{n, i, m, j}}$, and consider the family $A$ of commuting squares

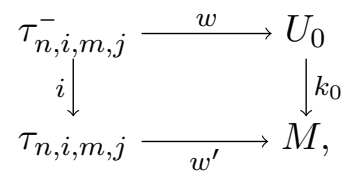

where $i$ is the inclusion. Define then $U$ and $k$ by pushout as in

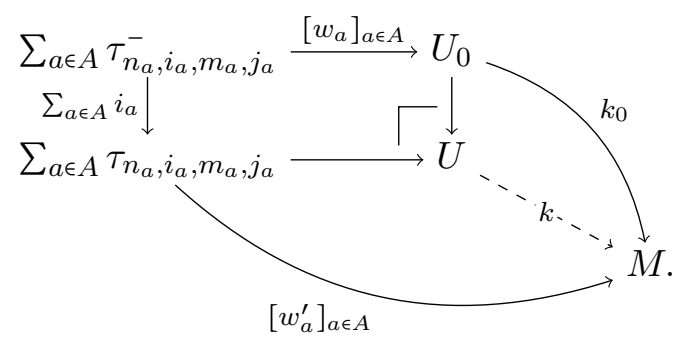

Informally, $U$ is $U_{0}$, where we add all the $\tau_{n, i, m, j}$ 's that exist in $M$ and whose horn is in $U_{0}$. We have by construction $\operatorname{El}(U)=\operatorname{El}\left(U_{0}\right)$, so $p^{\prime}$ is indeed a left inverse to $t^{\prime}: \operatorname{El}\left(Y^{\prime}\right) \rightarrow \operatorname{El}(U)$.

Finally, define $X^{\prime}, h$, and $s^{\prime}$ by the pullback

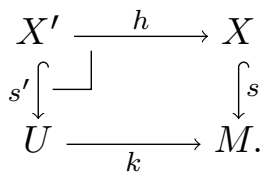

This altogether yields a vertical morphism

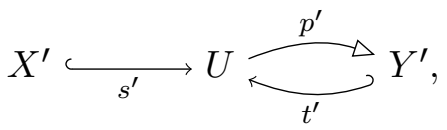

in $\mathbb{D}_{v}^{0}$. A tedious case analysis (made less tedious by $l: Y^{\prime} \hookrightarrow Y$ being monic) shows that, because $M$ is a move, $U$ is either a move or isomorphic to $Y^{\prime}$. So it is in $\mathbb{D}_{v}$. $U$ is our candidate cartesian lifting of $M$ along $l$. More generally, for any play $X \stackrel{s}{\rightarrow} U \stackrel{t}{\rightarrow} Y$, choose a decomposition into moves. We obtain a candidate cartesian lifting $X^{\prime} \stackrel{s^{\prime}}{\longrightarrow} U^{\prime} \stackrel{t^{\prime}}{\longrightarrow} Y^{\prime}$ for $U$, with morphism $(h, k, l)$ to $U$, along any $l: Y^{\prime} \hookrightarrow Y$ by taking the successive candidates for each move in the obvious way, and composing them.

To show that this indeed yields a cartesian lifting, consider any vertical morphism $X^{\prime \prime} \stackrel{s^{\prime \prime}}{\longrightarrow} U^{\prime \prime} \stackrel{t^{\prime \prime}}{\longrightarrow} Y^{\prime \prime}$ and diagram 


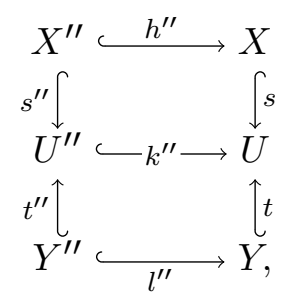

together with a map $l^{\prime}: Y^{\prime \prime} \rightarrow Y^{\prime}$ such that $l \circ l^{\prime}=l^{\prime \prime}$. By Proposition 7.26, letting $p^{\prime \prime}$ be the history of $U^{\prime \prime}$, the diagram

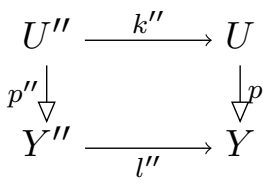

commutes, so by universal property of pullback, we obtain a map $k_{0}^{\prime}: \operatorname{El}\left(U^{\prime \prime}\right) \rightarrow \operatorname{El}\left(U^{\prime}\right)$, such that $k_{0} \circ k_{0}^{\prime}=k_{0}^{\prime \prime}$, where $k_{0}^{\prime \prime}$ is the restriction of $k^{\prime \prime}$ to dimensions $<4$. Furthermore, the expected map $k^{\prime}: U^{\prime \prime} \rightarrow U^{\prime}$, is given by universal property of pushout in

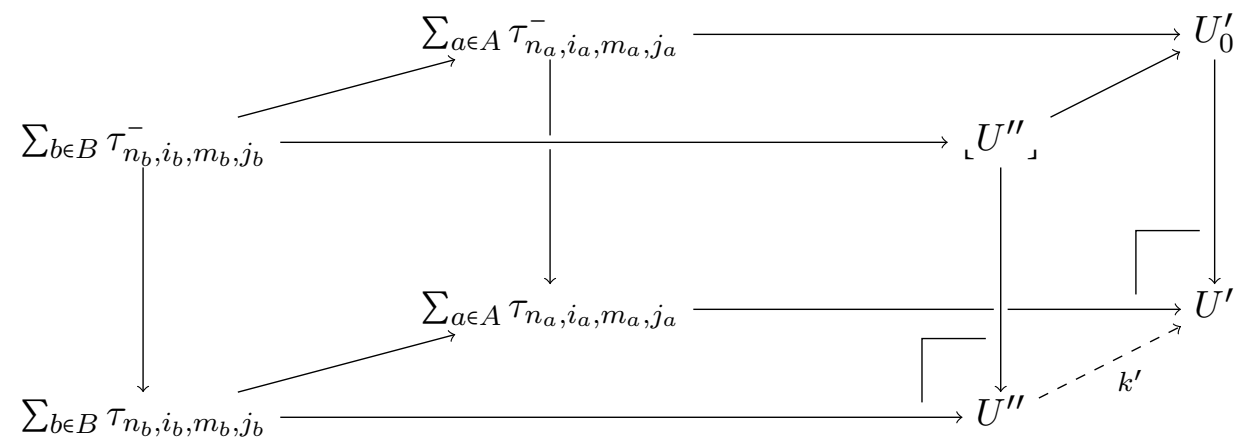

where $B$ is the family of all commuting squares

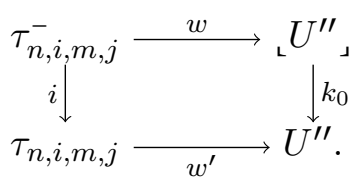

Finally, the desired map $h^{\prime}: X^{\prime \prime} \rightarrow X^{\prime}$ follows from universal property of $X^{\prime}$ as a pullback, and the square

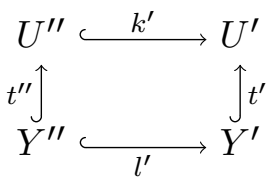

commutes by uniqueness in the universal property of $U^{\prime}$ as a pullback. 
7.4. Towards CCS as a playground. In this section, we prove an intermediate result for proving the decomposition axioms.

Consider a double cell $\alpha$ of the shape

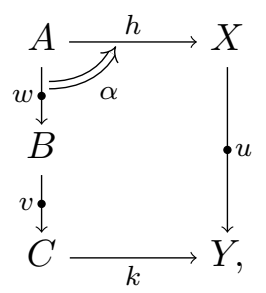

where $v$ is a view. Let now $\mathrm{D}_{\alpha}$ denote the category with

- objects all tuples $T=\left(Z, l, u_{1}, u_{2}, \alpha_{1}, \alpha_{2}, \alpha_{3}\right)$ such that

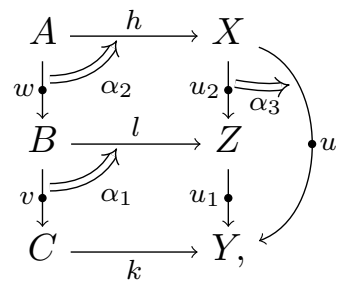

equals $\alpha$ and $\alpha_{3}$ is an isomorphism;

- with morphisms $T \rightarrow T^{\prime}$ given by tuples $(U, f, \beta, \gamma, \delta)$ (where $f$ is vertical) such that

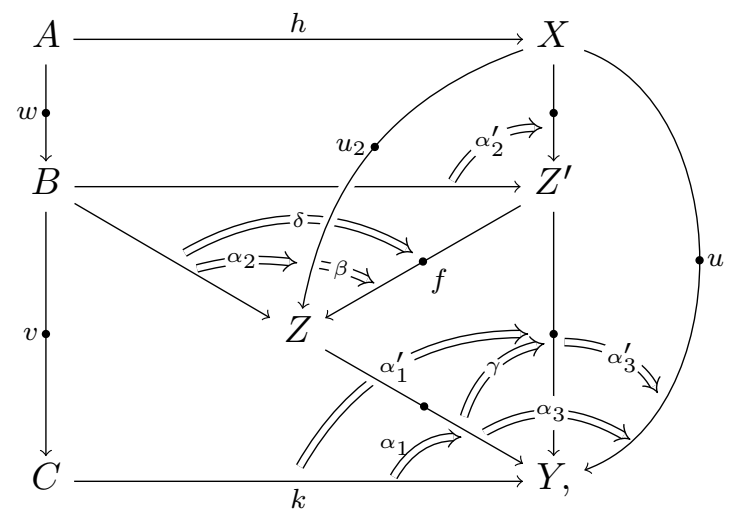

commutes, i.e., $\gamma \circ\left(\alpha_{1} \bullet \delta\right)=\alpha_{1}^{\prime}, \beta \circ \alpha_{2}=\delta \bullet \alpha_{2}^{\prime}$, and $\alpha_{3}^{\prime} \circ\left(\gamma \bullet u_{2}^{\prime}\right) \circ\left(u_{1} \bullet \beta\right)=\alpha_{3}$, and $\beta$ and $\gamma$ are isomorphisms;

- composition and identities are obvious.

So, objects of $\mathrm{D}_{\alpha}$ are decompositions of $u$ permitting corresponding decompositions of $\alpha$. The rest of this section is a proof of:

Lemma 7.28. $\mathrm{D}_{\alpha}$ has a weak initial object, i.e., an object $T$ such that for any object $T^{\prime}$ there is a morphism $T \rightarrow T^{\prime}$.

We start by extending the assignment $U \mapsto G_{U}$ to a functor, at least for source-linear $U$. Let SLin denote the full subcategory of $\widehat{\mathbb{C}}$ spanning source linear presheaves. The assignment $U \mapsto G_{U}$ actually extends to a functor $G_{-}: \operatorname{SLin} \rightarrow \mathrm{Gph} / L$, as follows. Let, first, for any move $x \in U$, the core associated to $x$, core $(x)$, be the unique core reachable from $x$ in $\int U$, i.e., the unique core $\mu$ for which there exists $f$ in $\mathbb{C}$ such that $\mu \cdot f=x$. Now, for any $\alpha: U \rightarrow U^{\prime}$ in $\widehat{\mathbb{C}}$, let $G_{\alpha}: G_{U} \rightarrow G_{U^{\prime}}$ map any core $x$ in $G_{U}$ to $\operatorname{core}(\alpha(x)) \in G_{U^{\prime}}$, and any non-core vertex 
$x \in G_{U}$ to $\alpha(x) \in G_{U^{\prime}}$. By naturality, this indeed defines a unique morphism of simple graphs over $L$.

Proposition 7.29. $G_{-}: \mathrm{SLin} \rightarrow \mathrm{Gph} / L$ is a functor.

We continue with some properties of $\mathbb{D}$.

Definition 7.30. A filiform play is any play $U$ such that the restriction of $G_{U}$ to cores and players is a filiform graph, i.e., a graph of the shape $\cdot \rightarrow \cdot \rightarrow \cdots$

E.g., all views are filiform.

Lemma 7.31. Any epimorphic (in $Đ$, hence isomorphic) double cell

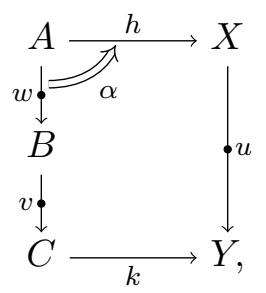

where $v$ is filiform decomposes as

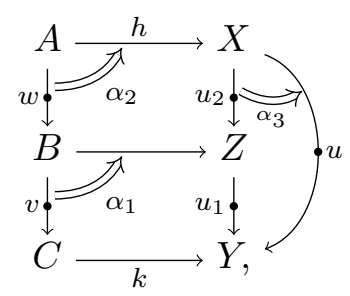

with $\alpha_{3}$ an isomorphism, $\alpha_{1}$ and $\alpha_{2}$ epimorphic, uniquely up to isomorphism. In this case, $u_{1}$ is filiform.

Proof. $B$ has just one player, say $b$. Let $b^{\prime}=\alpha(b)$. Because $\alpha$ is epi, $\alpha$ induces a morphism $G_{\alpha}: G_{v \bullet w} \rightarrow G_{u}$ of graphs, which is also epi. So, $G_{u}$ may be decomposed as a pushout

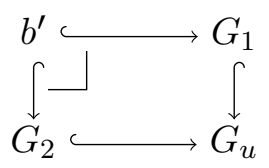

with $G_{1}=\operatorname{Im}_{G_{\alpha}}\left(G_{v}\right)$ and $G_{2}=\operatorname{Im}_{G_{\alpha}}\left(G_{w}\right)$. From this one deduces a decomposition of $u$ and $\alpha$.

Lemma 7.32. For any vertically composable $\alpha$ and $\beta$, if $\alpha \bullet \beta$ is epi, then so are $\alpha$ and $\beta$. Proof. Easy. 
Proof of Lemma 7.28. The double cell $\alpha$ induces morphisms of graphs $G_{v} \rightarrow G_{u} \leftarrow G_{w}$, by Proposition 7.29, Let

$$
u_{1}=\bigcap\left\{u^{\prime} \subseteq u \mid\left(Y \subseteq u^{\prime}\right) \wedge\left(\operatorname{Im}_{\alpha}\left(G_{v}\right) \subseteq G_{u^{\prime}}\right)\right\} .
$$

Thus, $v \rightarrow u$ factors as $v \rightarrow u_{1} \rightarrow u$. Let $Z$ be the position containing all channels of $u_{1}$, and all final players of $u_{1}$. Further let $\uparrow Z$ denote the full subgraph of $G_{u}$ containing all vertices $x$ with a path to some vertex of $Z$. Let then

$$
u_{2}=\bigcap\left\{u^{\prime \prime} \subseteq u \mid G_{u^{\prime \prime}} \supseteq \uparrow Z\right\} .
$$

The union $u_{1} \cup u_{2}$ is $u$, i.e., the square

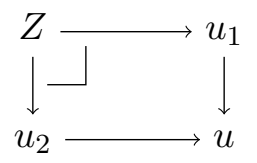

is a pushout, i.e., $u_{2} \bullet u_{1} \cong u$ in $\operatorname{Cospan}\left(\widehat{\mathbb{C}}^{f}\right)$. So it only remains to prove that $Z \rightarrow u_{1} \leftarrow X$ and $Y \rightarrow u_{2} \leftarrow Z$ are plays, for which we use Theorem [7.17. First, $u_{1}$ and $u_{2}$, as subpresheaves of $u$, both are locally 1-injective. Furthermore, $G_{u_{1}}$ and $G_{u_{2}}$, as subgraphs of a linear and acyclic graph, are also linear and acyclic. Now, by definition of $Z, Z \rightarrow u_{1}$ contains all channels and the final players of $u_{1}$. Further, since $X \subseteq u_{1}$, being initial in $u$ implies being initial in $u_{1}$, so $Z \rightarrow u_{1} \leftarrow X$ indeed is a play. Symmetrically, no player of $u_{1}$ not in $Z$ is final, so $Y \subseteq u_{2}$, and hence $Y \rightarrow u_{2}$ indeed contains all channels and final players. Finally, the players and channels of $Z$ are precisely the initial players and channels of $u_{2}$.

It remains to show that the induced decomposition of $\alpha$ is weakly initial. But any decomposition, inducing a decomposition $u_{1}^{\prime} \bullet u_{2}^{\prime}$ of $u$, should satisfy $Y \subseteq u_{1}^{\prime}, \operatorname{Im}_{\alpha}\left(G_{v}\right) \subseteq G_{u_{1}^{\prime}}$, and $G_{u_{2}^{\prime}} \subseteq \uparrow Z$, so, ignoring isomorphisms for readability, $u_{1} \subseteq u_{1}^{\prime}$ and $u_{2}^{\prime} \subseteq u_{2}$, as desired.

7.5. CCS as a playground. We are now ready to prove the decomposition axioms, which entail Theorem [7.3. They are proved in Lemmas 7.35 and 7.34 below.

Let us start with the following easy lemma.

Lemma 7.33. If $u=u_{2} \bullet u_{1}$, then, in $G_{u}$

- no player of $u_{1}$ is reachable from any core of $u_{2}$;

- no core of $u_{1}$ is reachable from any element of $u_{2}$.

Proof. For the first point, cores of $u_{2}$ only reach initial channels of $u_{1}$.

For the second point, we further observe that channel and players of $u_{2}$ only reach initial players and channels of $u_{1}$, hence no core.

The easiest decomposition axiom is (P8).

Lemma 7.34. $\mathbb{D}$ satisfies (P8).

Proof. Although the statement is complicated, this is rather easy: $\alpha$ restricts to a map of presheaves $f: b \rightarrow(M \bullet u)$, on which we proceed by case analysis.

If $\operatorname{Im}(f) \subseteq M$, then by Lemma 7.28 and correctness we are in the left-hand case. Otherwise, assume that a move $\mu^{\prime} \in M$ is in the image of $\alpha$, say of a move $\mu \in w$. We have a path $\mu \rightarrow b$ in $b \bullet w$, hence a path core $\left(\mu^{\prime}\right) \rightarrow \alpha(b)$ in $M \bullet u$, contradicting Lemma 7.33 . 
Let us now attack the last axiom.

Lemma 7.35. $\mathbb{D}$ satisfies $\left(P^{7}\right)$.

We need a few lemmas.

Lemma 7.36. For any plays $A \stackrel{u_{1}}{\rightarrow} B \stackrel{u_{2}}{\rightarrow} C$, for any player or channel $x \in u_{2}$ and core $\mu \in u_{1}$, there is no edge $x \rightarrow \mu$ in $u_{2} \bullet u_{1}$.

Proof. The existence of $e: x \rightarrow \mu$ implies $x \in B$, hence $x$ initial in $u_{1}$, which contradicts the very existence of $e$.

Lemma 7.37. Morphisms of plays preserve finality.

Proof. If a player is final in the domain, then it is in the final position, hence has an image in the final position of the codomain, hence is final there.

Lemma 7.38. For any map $\alpha: u \rightarrow w$ in $Đ$, for any player $x$ in $u$ and edge $e^{\prime}: \mu^{\prime} \rightarrow \alpha(x)$ from a core in $w$, there exists a core $\mu \in u$ and an edge $e: \mu \rightarrow x$ in $u$ such that $G_{\alpha}(e)=e^{\prime}$.

Proof. Let first $X \rightarrow u \leftarrow Y$ and $X^{\prime} \rightarrow w \leftarrow Y^{\prime}$ be the considered morphisms.

Then, observe that $x$ is not final in $u$, for otherwise it would be in $X$, hence $\alpha(x)$ would be in $X^{\prime}$ and final, contradicting the existence of $e^{\prime}$.

So there exists $e: \mu \rightarrow x$ in $u$. But now, by target-linearity, $G_{\alpha}(\mu)=\mu^{\prime}$, which entails the result.

Lemma 7.39. In any double cell (7.1), both squares are pullbacks.

Proof. $X$ must consist precisely of all final players and channels of $G_{U}$, which must also be final in $G_{V}$, so finality in $G_{U}$ implies finality in $G_{V}$. Conversely, any player or channel mapped to a final one in $G_{V}$ has to be final. So $X$ is a pullback of $U$ and $X^{\prime}$. The lower square being a pullback follows from similar reasoning.

Proof of Lemma 7.35. Consider any $\alpha$, and construct $C, u_{1}, u_{2}$, and the morphisms in Figure 5. as follows. First, let $u_{1}$ be the pullback $u \times_{w} w_{1}$, and then $C=u_{1} \times_{w_{1}} Y$. Let then $u_{2}=u \times_{w} w_{2}$, and the arrow $C \rightarrow u_{2}$ be induced by universal property of pullback. By the pullback lemma, $C=u_{2} \times_{w_{2}} Y$. Because presheaf categories are adhesive [32], $\widehat{\mathbb{C}}^{f}$ is, and, $Y \rightarrow w_{1}$ being monic, we have a Van Kampen square. Thus, by the main axiom for adhesive categories, $u$ is a pushout $u_{1}+_{C} u_{2}$, i.e., $u \cong u_{2} \bullet u_{1}$ in Cospan $\left(\widehat{\mathbb{C}}^{f}\right)$. Letting $\alpha_{i}$ be the arrow $u_{i} \rightarrow w_{i}$, for $i=1,2$, this yields the desired decomposition of $\alpha$.

We still need to show that $A \rightarrow u_{1} \leftarrow C$ and $C \rightarrow u_{2} \leftarrow B$ are plays, and that the obtained decomposition is unique. Uniqueness follows from adhesivity of $\widehat{\mathbb{C}}$ and Lemma 7.39. Indeed, any decomposition looks like Figure 5 , except that $u_{1}, u_{2}$, and $C$ are not a priori obtained by pullback. But by Lemma 7.39, both back faces have to be pullbacks, hence so are the front faces by adhesivity.

Let us finally show that $u_{1}$ and $u_{2}$ are plays. It is easy to see that non-linearity or non-acyclicity of $G_{u_{1}}$ (resp. $G_{u_{2}}$ ) would entail non-linearity or non-acyclicity of $u$ or $w_{1}$ (resp. or $w_{2}$ ). Local 1-injectivity is also easy.

Let us now prove the missing conditions for $A \rightarrow u_{1} \leftarrow C$.

a) Any player $x$ of $u_{1}$ in the image of $A$ is final, for otherwise its image in $w_{1}$ would be in the image of $X$ and non-final. 


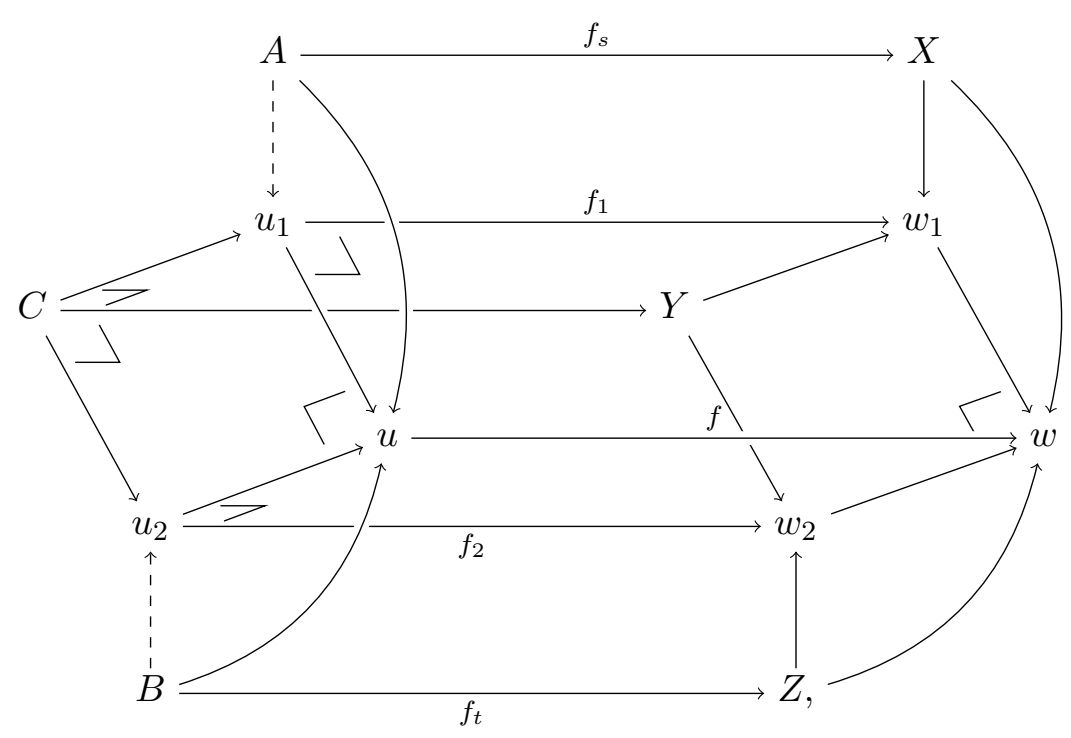

Figure 5: Proof of Lemma 7.35

b) Conversely, if a player $x \in u_{1}$ is final but not in $A$, then its image in $u$ must be non-final by Theorem 7.17, because $u_{1} \rightarrow u$ is monic. But then there is a core $\mu$ of $u_{2}$ with a path $\mu \rightarrow x$ in $G_{u}$, whose images in $w$ yield a path from a core of $w_{2}$ to a player of $w_{1}$, contradicting Lemma 7.33. So $A$ contains precisely the final players of $u_{1}$.

c) Now, if a channel $x \in u_{1}$ is not in $A$, then its image in $u$ must be in $A$, hence $u_{1} \rightarrow u$ cannot be mono, so neither can $w_{1} \rightarrow w$, so neither can $Y \rightarrow w_{2}$, contradiction.

d) Finally, by construction, $C$ contains precisely the initial players and channels of $u_{1}$.

Now, for $C \rightarrow u_{2} \leftarrow B$.

a) By universal property of pullback, $C$ contains all channels of $u_{2}$.

b) For players, clearly, for any player $x$ in $C, x$ is final in $u_{2}$. Indeed, otherwise, there would be a path $\mu \rightarrow x$ from a core $\mu$ in $u_{2}$, yielding a path $f_{2}(\mu) \rightarrow f_{2}(x)$ in $w_{2}$. But since $x$ is in $C, f_{2}(x) \in Y$, which hence contains a non-final player, contradiction.

c) Conversely, if $x$ is final in $u_{2}$, then $x^{\prime}=f_{2}(x)$ is final in $w_{2}$. Indeed, otherwise, there would be an edge $\mu^{\prime} \rightarrow x^{\prime}$ from a core in $w_{2}$, so, by Lemma 7.38, an edge $\mu \rightarrow x$ in $u$ with $f(\mu)=\mu^{\prime}$. But then, $\mu \in u_{2}$, so $x$ cannot be final. This shows that $x^{\prime}$ is final in $w_{2}$. But then $x^{\prime} \in Y$, so, because $C=u_{2} \times_{w} Y, x \in C$.

d) Consider now any player or channel $x$ initial in $u_{2}$. First, $x$ is also initial in $u$ : otherwise, there would be an edge $x \rightarrow \mu$ to a core in $u$, with $\mu \in u_{1}$, hence an edge $f(x) \rightarrow f(\mu)$ in $w$ from a channel of $w_{2}$ to a core of $w_{1}$, which is impossible by Lemma 7.36. So $x$ is initial in $u$, hence $x \in B$.

e) Now, for any player or channel $x \in B, x$ is initial in $u$, hence $x$ is a fortiori initial in $u_{2}$. 


\section{Conclusion And Perspectives}

8.1. Conclusion. We have described a denotational semantics of CCS based on presheaves, with a strong game-semantical flavour. Some aspects of the approach look promising to us.

First, our result is encouraging for potential applications of Kleene coalgebra to programming language theory, i.e., ascribing a semantics to the 'rule of the game' rather than attempting to organise operational semantics into some categorical structure.

Second, our use of techniques from categorical combinatorics (e.g., defining positions and plays as finite presheaves) provide a high-level, yet rigorous toolbox for dealing with string diagrams. (Compare, e.g., with available definitions of linear logic proof nets or interaction nets.)

Third, our notion of play encompassing both views and closed-world plays, and its rich notion of morphism yields a convincing interplay between strategies (presheaves on views) and behaviours (presheaves on plays). In particular,

- passing from one to the other is handled by standard categorical constructions,

- the general syntax and LTS for strategies provides a link to syntactic approaches.

Other aspects of our model are not as satisfactory.

First of all, the notion of playground is very complicated. In work in progress on a similar approach for $\pi$-calculus, we bypass the intermediate LTS $\mathcal{T}_{\mathbb{D}}$ of process terms, because it does not help so much - strategies are already really close to $\pi$-calculus terms. This seems to hint that the main result of playground theory is actually the characterisation of strategies by the syntax of Section 5.1. The good point is: this result does not at all need all axioms for playgrounds.

A second negative point is that some proofs may probably be improved. E.g., our proof that $\theta: C C S \rightarrow \mathcal{T}_{\mathbb{D}} C C S$ is included in weak bisimilarity is a bit of a nightmare, with no apparent good reason. Similarly, we know already that our constructions for showing the fibration axiom (P1) may be improved. Indeed, the trick we use to restore synchronisations after restriction rests upon a factorisation system [16, 28]. In our current work on $\pi$, we use factorisation systems to prove the fibration axiom in a much more direct way (which was prompted by the fact that the method used here does not apply).

8.2. Perspectives. Beyond these rather technical concerns, we plan to adapt our semantics to more complicated calculi like $\pi$, the Join and Ambients calculi, calculi with passivation, functional calculi, possibly with extra features (e.g., references, data abstraction, encryption), with a view to eventually generalising it, perhaps to some SOS format. In particular, adapting the approach to functional calculi should clarify the relationship with Hyland-Ong innocence. In work in progress mentioned above, we construct a playground for $\pi$, whose proof of full abstraction remains to be completed. More speculative directions include

- designing a general way of constructing playgrounds automatically from more elementary data; work in progress reveals that this is a very subtle task;

- defining a notion of morphisms for playgrounds, which should induce translation functions between strategies, and find sufficient conditions for such morphisms to preserve, resp. reflect testing equivalences;

- generalising playgrounds to apply them beyond programming language semantics; in particular, preliminary work shows that playgrounds easily account for cellular automata; 
this raises the question of how morphisms of playgrounds would compare with various notions of simulations between cellular automata [10];

- incorporate quantitative aspects from Kleene coalgebra into playground theory; this may start by refining fair testing equivalence to keep track of the probability of passing each test successfully.

\section{REFERENCES}

[1] Samson Abramsky and Paul-André Melliès. Concurrent games and full completeness. In LICS 1999 [37], pages 431-442. doi: 10.1109/LICS.1999.782638.

[2] Samson Abramsky, Radha Jagadeesan, and Pasquale Malacaria. Full abstraction for PCF. Information and Computation, 163(2):409-470, 2000. doi: 10.1006/inco.2000. 2930.

[3] J. Adámek, J.; Rosicky. Locally Presentable and Accessible Categories. Cambridge University Press, 1994. doi: 10.1017/CBO9780511600579.

[4] Gérard Berry and Gérard Boudol. The chemical abstract machine. In POPL, pages 81-94, 1990. doi: 10.1145/96709.96717.

[5] Filippo Bonchi, Marcello M. Bonsangue, Jan J. M. M. Rutten, and Alexandra Silva. Deriving syntax and axioms for quantitative regular behaviours. In CONCUR, volume 5710 of $L N C S$, pages 146-162. Springer Verlag, 2009. doi: 10.1007/978-3-642-04081-8_ 11.

[6] Marcello M. Bonsangue, Jan J. M. M. Rutten, and Alexandra Silva. A Kleene theorem for polynomial coalgebras. In FoSSaCS, volume 5504 of $L N C S$, pages 122-136. Springer Verlag, 2009. doi: 10.1007/978-3-642-00596-1_10.

[7] Diletta Cacciagrano, Flavio Corradini, and Catuscia Palamidessi. Explicit fairness in testing semantics. Logical Methods in Computer Science, 5(2), 2009. doi: 10.2168/ LMCS-5(2:15)2009.

[8] Simon Castellan, Pierre Clairambault, and Glynn Winskel. Concurrent Hyland-Ong games. GaLoP, 2014.

[9] Rocco De Nicola and Matthew Hennessy. Testing equivalences for processes. Theoretical Computer Science, 34:83-133, 1984. doi: 10.1016/0304-3975(84)90113-0.

[10] Marianne Delorme, Jacques Mazoyer, Nicolas Ollinger, and Guillaume Theyssier. Bulking I: An abstract theory of bulking. Theoretical Computer Science, 412(30):3866-3880, 2011. doi: $10.1016 /$ j.tcs.2011.02.023.

[11] Charles Ehresmann. Catégories structurées. Annales scientifiques de l'Ecole Normale Supérieure, 80(4):349-426, 1963.

[12] Charles Ehresmann. Catégories et structures. Dunod, 1965.

[13] Claudia Faggian and Mauro Piccolo. Partial orders, event structures and linear strategies. In TLCA, volume 5608 of $L N C S$, pages 95-111. Springer Verlag, 2009. doi: 10.1007/978-3-642-02273-9_9.

[14] Marcelo P. Fiore. Fibred models of processes: Discrete, continuous, and hybrid systems. In IFIP TCS, volume 1872 of LNCS, pages 457-473. Springer Verlag, 2000. doi: 10. 1007/3-540-44929-9_32.

[15] FoSSaCS 2004. FoSSaCS, volume 2987 of $L N C S$, 2004. Springer Verlag.

[16] Peter Freyd and G. M. Kelly. Categories of continuous functors, I. Journal of Pure and Applied Algebra, 2:169-191, 1972. doi: 10.1016/0022-4049(72)90001-1.

[17] Richard H. G. Garner. Polycategories. PhD thesis, University of Cambridge, 2006. 
[18] Dan R. Ghica and Andrzej S. Murawski. Angelic semantics of fine-grained concurrency. In FoSSaCS 2004 [15], pages 211-225. doi: 10.1007/978-3-540-24727-2_16.

[19] Daniele Gorla. Towards a unified approach to encodability and separation results for process calculi. Information and Computation, 208(9):1031-1053, 2010. doi: 10.1016/ j.ic.2010.05.002.

[20] Marco Grandis and Robert Paré. Limits in double categories. Cahiers de Topologie et Géométrie Différentielle Catégoriques, 40(3):162-220, 1999.

[21] Marco Grandis and Robert Paré. Adjoints for double categories. Cahiers de Topologie et Géométrie Différentielle Catégoriques, 45(3):193-240, 2004.

[22] Russell Harmer and Guy McCusker. A fully abstract game semantics for finite nondeterminism. In LICS 1999 [37], pages 422-430. doi: 10.1109/LICS.1999.782637.

[23] Russell Harmer, Martin Hyland, and Paul-André Melliès. Categorical combinatorics for innocent strategies. In LICS, pages 379-388. IEEE Computer Society, 2007. doi: 10.1109/LICS.2007.14.

[24] Tom Hirschowitz and Damien Pous. Innocent strategies as presheaves and interactive equivalences for CCS. In ICE, pages 2-24, 2011. doi: 10.4204/EPTCS.59.2.

[25] Tom Hirschowitz and Damien Pous. Innocent strategies as presheaves and interactive equivalences for CCS. Scientific Annals of Computer Science, 22(1):147-199, 2012. doi: 10.7561/SACS.2012.1.147. Selected papers from ICE' '11.

[26] J. M. E. Hyland and C.-H. Luke Ong. On full abstraction for PCF: I, II, and III. Inf. Comput., 163(2):285-408, 2000. doi: 10.1006/inco.2000.2917.

[27] Bart Jacobs. Categorical Logic and Type Theory. Number 141 in Studies in Logic and the Foundations of Mathematics. North Holland, Amsterdam, 1999.

[28] André Joyal. Factorisation systems. http://ncatlab.org/joyalscatlab.

[29] André Joyal and Myles Tierney. Notes on simplicial homotopy theory. Course at the CRM, February 2008.

[30] André Joyal, Mogens Nielsen, and Glynn Winskel. Bisimulation and open maps. In LICS, pages 418-427. IEEE Computer Society, 1993. doi: 10.1109/LICS.1993.287566.

[31] Joachim Kock. Polynomial functors and trees. International Mathematics Research Notices, 2011(3):609-673, 2011. doi: 10.1093/imrn/rnq068.

[32] Stephen Lack and Pawel Sobocinski. Adhesive categories. In FoSSaCS 2004 [15], pages 273-288. doi: 10.1007/978-3-540-24727-2_20.

[33] James Laird. Game semantics for higher-order concurrency. In FSTTCS, volume 4337 of LNCS, pages 417-428. Springer Verlag, 2006. doi: 10.1007/11944836_38.

[34] F. William Lawvere and Stephen H. Schanuel. Conceptual mathematics - a first introduction to categories. Cambridge University Press, 1997.

[35] Tom Leinster. Higher Operads, Higher Categories, volume 298 of London Mathematical Society Lecture Notes. Cambridge University Press, Cambridge, 2004.

[36] Tom Leinster. Basic Category Theory, volume 143 of Cambridge Studies in Advanced Mathematics. Cambridge University Press, 2014.

[37] LICS 1999. 14th Symposium on Logic in Computer Science, 1999. IEEE Computer Society.

[38] Saunders Mac Lane. Categories for the Working Mathematician. Number 5 in Graduate Texts in Mathematics. Springer Verlag, 2nd edition, 1998.

[39] Saunders MacLane and Ieke Moerdijk. Sheaves in Geometry and Logic: A First Introduction to Topos Theory. Universitext. Springer, 1992. 
[40] Paul-André Melliès. Asynchronous games 2: the true concurrency of innocence. In Proc. CONCUR '04, volume 3170 of LNCS, pages 448-465. Springer Verlag, 2004. doi: 10.1007/978-3-540-28644-8_29.

[41] Paul-André Melliès. Game semantics in string diagrams. In LICS, pages 481-490. IEEE, 2012. doi: .1109/LICS.2012.58.

[42] Paul-André Melliès and Samuel Mimram. Asynchronous games: Innocence without alternation. In CONCUR, volume 4703 of $L N C S$, pages 395-411. Springer Verlag, 2007. doi: 10.1007/978-3-540-74407-8_27.

[43] Robin Milner. A Calculus of Communicating Systems, volume 92 of LNCS. Springer, 1980. doi: 10.1007/3-540-10235-3.

[44] Robin Milner. Communication and Concurrency. Prentice-Hall, 1989.

[45] Hanno Nickau. Hereditarily sequential functionals. In LFCS, volume 813 of LNCS, pages 253-264. Springer Verlag, 1994. doi: 10.1007/3-540-58140-5_25.

[46] Robert Paré. Yoneda theory for double categories. Theory and Applications of Categories, 25(17):436-489, 2011.

[47] Julian Rathke and Pawel Sobocinski. Deconstructing behavioural theories of mobility. In IFIP TCS, volume 273 of IFIP, pages 507-520. Springer Verlag, 2008. doi: 10.1007/ 978-0-387-09680-3_34.

[48] Arend Rensink and Walter Vogler. Fair testing. Information and Computation, 205(2): 125-198, 2007. doi: 10.1016/j.ic.2006.06.002.

[49] Silvain Rideau and Glynn Winskel. Concurrent strategies. In LICS, pages 409-418. IEEE Computer Society, 2011. doi: 10.1109/LICS.2011.13.

[50] Davide Sangiorgi. Introduction to Bisimulation and Coinduction. Cambridge University Press, 2012.

[51] Davide Sangiorgi and Jan Rutten, editors. Advanced Topics in Bisimulation and Coinduction. Number 52 in Cambridge Tracts in Theoretical Computer Science. Cambridge University Press, 2011.

[52] Davide Sangiorgi and David Walker. The $\pi$-calculus - a theory of mobile processes. Cambridge University Press, 2001.

[53] Glynn Winskel. Strategies as profunctors. In FoSSaCS, volume 7794 of $L N C S$, pages 418-433. Springer Verlag, 2013. doi: 10.1007/978-3-642-37075-5_27.

[54] Glynn Winskel and Mogens Nielsen. Handbook of Logic in Computer Science, volume 4 of Oxford science publications, chapter Models for concurrency. Clarendon, 1995.

This work is licensed under the Creative Commons Attribution-NoDerivs License. To view a copy of this license, visit http://creativecommons.org/licenses/by-nd/2.0/ or send a letter to Creative Commons, 171 Second St, Suite 300, San Francisco, CA 94105, USA, or Eisenacher Strasse 2, 10777 Berlin, Germany 


\begin{tabular}{|c|c|c|c|}
\hline $\mathbb{C}$ & base category, over which positions & $\perp^{G}$ & pole for fair testing eq. in $G$ \\
\hline & and plays are presheaves & $\Perp$ & pole for semantic fair test. eq. \\
\hline$\star,[n]$ & objects of $\mathbb{C}$ & $\perp$ & pole for CCS (Def. 2.22) \\
\hline$\pi_{n}^{l}, \pi_{n}^{r}, \pi_{n}$ & & $\mathcal{L}$ & intermediate pole (Lem. 6.22) \\
\hline$\nu_{n}, \nabla_{n}, \iota_{n, i}$ & & $C C S$ & LTS for CCS \\
\hline$o_{m, j}, \tau_{n, i, m, j}$ & & $\mathcal{S}$ & LTS for strategies \\
\hline$[m]_{a_{1}}, \ldots c_{1}, \ldots[n]$ & two players sharing some channels & $\mathrm{T}$ & erms \\
\hline Cospan $(-)$ & bicategory of cospans of - & $\mathcal{T}$ & LTS for $T: o b(\mathcal{T})=T$ \\
\hline $\mathbb{D}_{h}$ & $\begin{array}{l}\text { category of positions and embed- } \\
\text { dings }\end{array}$ & $\begin{array}{l}0-1 \\
\theta\end{array}$ & $\begin{array}{l}\text { translation } C C S \rightarrow \mathcal{S} \\
\text { translation } C C S \rightarrow \mathcal{T}\end{array}$ \\
\hline $\mathbb{D}_{v}$ & bicategory of positions and plays & $\llbracket-\rrbracket$ & translation $\mathcal{T} \rightarrow \mathcal{S}$ \\
\hline $\mathbb{D}$ & playground & $\mathbb{W}_{C C S}$ & set of closed-world quasi-moves \\
\hline $\mathbb{D}^{C C S}$ & playground for CCS & $\mathbb{D}^{\mathbb{W}} \subseteq \mathbb{D}_{v}$ & subbicat. of closed-world plays \\
\hline $\mathbb{E}$ & category of plays and extensions & $\ell_{\mathbb{D}}$ & labelling of closed-world plays \\
\hline $\mathrm{B}_{X}$ & category of behaviours on $X$ & & in $\{i d, \varnothing\}: \mathbb{D}^{\mathbb{W}} \rightarrow \mathrm{fc}(\Sigma)$ \\
\hline $\mathbb{E}^{\mathbb{V}}$ & category of views and extensions & $A^{\mathbb{W}}$ & 'closed-world' subgraph of a \\
\hline$S S_{X}$ & category of strategies on $X$ & & graph with complementarity $A$ \\
\hline $\begin{array}{l}\mathrm{Pl}(X) \\
v^{x, u}\end{array}$ & $\begin{array}{l}\text { players of position } X \\
\text { view of } x: d \rightarrow X \text { in } u: X \rightarrow Y\end{array}$ & $\triangleright^{A}$ & $\begin{array}{l}\text { compatibility relation for } A \text { : } \\
A^{2} \mapsto A^{\mathbb{W}}\end{array}$ \\
\hline $\begin{array}{l}x^{u}: d^{x, u}: Y \\
u_{\mid k}: D_{k, u} \rightarrow Y\end{array}$ & $\begin{array}{l}\text { initial player of } x \text { in } u \\
\text { restriction of } u: X^{\prime} \rightarrow X \text { along }\end{array}$ & $e \Downarrow e^{\prime}$ & $\begin{array}{l}\text { notation for the composite } \\
A^{\complement} \hookrightarrow A^{2} \rightarrow A^{\mathbb{W}} \rightarrow \Sigma\end{array}$ \\
\hline $\mathrm{Pl}_{M}(X)$ & $\begin{array}{l}k: Y \rightarrow X \\
\text { players of position } X \text { whose view in } \\
M: X \rightarrow Y \text { is non-trivial }\end{array}$ & $\begin{array}{l}{[x, y]} \\
\chi: \mathbb{I} \rightarrow \mathbb{Q}\end{array}$ & $\begin{array}{l}\text { choice of 'amalgamation' in } G \\
\text { subgraph of edges with double } \\
\text { cell } i d_{I}^{\bullet} \rightarrow M\end{array}$ \\
\hline & projection of $S \in S S_{X}$ to $x \in \operatorname{Pl}(X)$ & $\xi: \mathbb{I} \mathbb{Q}$ & mapping to CCS labels \\
\hline$[S, T]$ & and $T$ & $G$ modular & $\triangleright^{G}$ strong bisim over $\Sigma$ \\
\hline$S \cdot v$ & of $S$ after $v$ & $x^{\circ}$ & $\{y \mid x \frown y\}$ \\
\hline & restriction of $S$ to antecedents of $\sigma$ & $x \bowtie y$ & $x^{\curvearrowright}=y^{\curvearrowright}$ \\
\hline$[\mathbb{B}]_{d}$ & $\begin{array}{l}\text { graph of full quasi-moves } \\
\text { set of isomorphism classes of basic }\end{array}$ & $G \diamond_{A} H$ & $\begin{array}{l}\text { blind composition of } G \text { and } H \\
\text { over } A\end{array}$ \\
\hline$[\mathbb{F}]_{X}$ & $\begin{array}{l}\text { moves over } d \\
\text { set of isomorphism classes of full } \\
\text { moves over } X\end{array}$ & $\begin{array}{l}\text { adequacy } \\
\text { of } G \rightarrow A \\
\mathcal{H}^{A}\end{array}$ & $\begin{array}{l}\text { (essentially) } \perp^{G^{\diamond}}=\perp^{G \diamond_{A} G} \\
A \text {-trees }\end{array}$ \\
\hline$\chi[M]$ & set of basic $b$ 's s.t. $\exists b \rightarrow M$ & $\mathcal{F}_{a}$ & failures over $a \in A$ \\
\hline$\left[\mathbb{F}^{1}\right]_{X} \subseteq[\mathbb{F}]_{X}$ & 11 moves $M$ such that & & failures to $A$-trees: $\mathcal{F} \rightarrow \mathcal{H}^{A}$ \\
\hline$\left[\mathbb{F}^{+}\right]_{X} \subseteq[\mathbb{F}]_{X}$ & $\begin{array}{l}\chi[M] \text { is a singleton } \\
\text { subset of full moves } M \text { such that }\end{array}$ & & $\begin{array}{l}\text { enough ticks, finitely branch- } \\
\text { ing, inertly silent (Def. 6.43) }\end{array}$ \\
\hline & ingleton & core & move element of some presheaf, \\
\hline$r^{u}, i^{u}$ & bijection, for all plays $u: X^{\prime} \rightarrow X$, & & of maximal dimension \\
\hline$d \vdash S$ & a & aj. & $\begin{array}{l}\text { cores map inj. to } U \text {, except per- } \\
\text { haps for channels in the inter- }\end{array}$ \\
\hline$d \vdash_{\mathrm{D}} D$ & tegy term & & \\
\hline$d \vdash T$ & process term & $G_{U}$ & causal graph of $U$ \\
\hline$(I, h, S)^{\perp}$ & set of tests passed by $(I, h, S)$ & $\mathrm{El}(-)$ & elements \synchronisations \\
\hline & fair testing eq. in graph w.c. $G$ & $\rightarrow$ & map between El(-)'s \\
\hline$\sim_{f, s}$ & fair testing eq. in CCS & horn $\tau$ & synchro. minus $i d_{\tau_{n, i, m, j}}$ \\
\hline & semantic fair testing eq. & $\Delta_{f}$ & change of base along $f$ \\
\hline
\end{tabular}

Figure 6: Cheat sheet 